NISTIR 8372

\title{
Study of 5G New Radio (NR) Support for Direct Mode Communications
}

Fernando J. Cintrón

David W. Griffith

Chunmei Liu

Richard Rouil

Yishen Sun

Jian Wang

Peng Liu

Chen Shen

Aziza Ben Mosbah

Samantha Gamboa

This publication is available free of charge from:

https://doi.org/10.6028/NIST.IR.8372

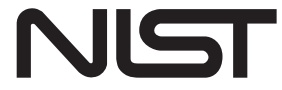

National Institute of Standards and Technology

U.S. Department of Commerce 


\section{NISTIR 8372}

\section{Study of 5G New Radio (NR) Support for Direct Mode Communications}

Fernando J. Cintrón

David W. Griffith

Chunmei Liu

Richard Rouil

Yishen Sun

Jian Wang

Wireless Networks Division

Communications Technology Laboratory

Peng Liu

Chen Shen

Associate, Wireless Networks Division

Communications Technology Laboratory

Department of Physics, Georgetown University, Washington, D.C.

Aziza Ben Mosbah

Samantha Gamboa

Associate, Wireless Networks Division

Communications Technology Laboratory

Prometheus Computing LLC, Sylva, North Carolina

This publication is available free of charge from:

https://doi.org/10.6028/NIST.IR.8372

May 2021

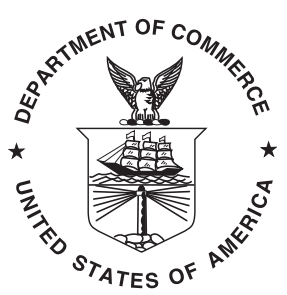

U.S. Department of Commerce Gina M. Raimondo, Secretary

National Institute of Standards and Technology James K. Olthoff, Performing the Non-Exclusive Functions and Duties of the Under Secretary of Commerce for Standards and Technology \& Director, National Institute of Standards and Technology 
Certain commercial entities, equipment, or materials may be identified in this document in order to describe an experimental procedure or concept adequately. Such identification is not intended to imply recommendation or endorsement by the National Institute of Standards and Technology, nor is it intended to imply that the entities, materials, or equipment are necessarily the best available for the purpose.

National Institute of Standards and Technology Interagency or Internal Report 8372

Natl. Inst. Stand. Technol. Interag. Intern. Rep. 8372, 82 pages (May 2021)

This publication is available free of charge from: https://doi.org/10.6028/NIST.IR.8372 


\begin{abstract}
In public safety communications, direct mode communication is essential to keep first responders connected, especially when there is no network coverage. Direct mode communication is supported in Third Generation Partnership Project (3GPP) Long Term Evolution (LTE) via Sidelink (SL). With 3GPP advancing from LTE to Fifth Generation (5G), in this study we explore new 3GPP 5G New Radio (NR) SL features and evaluate its support for direct mode communications.

This study focuses on five major topics that are essential to public safety communications over 5G New Radio (NR) SL: resource management, physical layer, capacity, Quality of Service (QoS), and LTE/NR coexistence. First, on resource management, we analyze the supported mapping between applications and SL resources, control of communication type (unicast, multicast, and broadcast), together with SL physical resource pools. We then identify several areas for further research, including resource provision for different types of applications and management of concurrent applications. Second, on physical layer, we highlight NR's new features that are expected to improve performance, including NR's flexible numerology, feedback channel, advanced channel coding mechanism, and channel sensing. Third, on capacity, we use maximal achievable data rate as the measure and analyze in detail the factors that impact NR SL capacity, including various parameters and overheads. We then study SL capacity under various NR operational configurations and device capabilities. Using Band n14 for numerical analyses, we also analyze LTE capacity for comparison purpose, and further quantify NR capacity improvements over LTE brought by each of the NR new features. Fourth, on QoS, we notice that compared with the relatively simplified LTE QoS operations, NR SL has much enhanced QoS support by inheriting various Uu QoS operations and by introducing new features, such as the new layer Service Data Application Protocol (SDAP) and the new parameter Range. The NR SL QoS parameters are also backwards compatible with QoS parameters of LTE Proximity Services (ProSe). We describe these enhancements in detail, as well as the ongoing 3GPP standardization efforts in supporting public safety communications. Fifth, on LTE/NR coexistence, we address the transition from LTE to NR before re-farming the full bands. We discuss in detail Dynamic Spectrum Sharing (DSS), such as the exchange of configurations between LTE and NR, then point out the limitations in supporting sharing over SL, and possible solutions in 3GPP standard efforts. Last but not least, we provide an overview of ongoing 3GPP study items and work items related to the NR SL, including NR ProSe, direct discovery, direct communication, and UE-based relays, as well as enhancements to NR SL power efficiency, reliability, and latency.

While our study shows the potential of a promising performance improvement on direct mode communications for 5G NR SL over LTE SL, the study also identifies its limitations. Addressing these limitations to better support first responder needs, plus developing measurement tools to quantify performance, will be our next steps.
\end{abstract}




\section{Key words}

5G New Radio; D2D; Direct Mode; Sidelink; Public Safety. 


\section{Table of Contents}

Definitions vii

Abbreviations vii

1 Executive Summary 1

2 Introduction $\quad 4$

3 Resource Management 6

$\begin{array}{lll}3.1 & \text { Introduction } & 6\end{array}$

3.2 V2X Services Provisioning 6

3.2.1 Authorization $\quad 7$

$\begin{array}{lll}3.2 .2 & \text { Service Configuration } & 7\end{array}$

3.2.3 Resource Provisioning 8

3.3 Sidelink Resource Allocation $\quad 8$

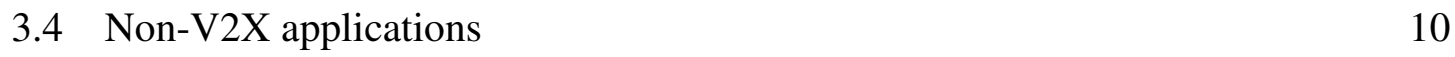

$\begin{array}{lll}3.5 & \text { Summary } & 10\end{array}$

4 Physical Layer $\quad 11$

4.1 Introduction 11

4.2 Frame Structure and Physical Resources 11

4.2.1 Numerologies, Frames, Subframes, and Slots 11

$\begin{array}{ll}\text { 4.2.2 Physical Resources } & 12\end{array}$

4.3 Sidelink Channels and Reference Signals 13

$\begin{array}{ll}\text { 4.3.1 Physical Sidelink Channels } & 14\end{array}$

$\begin{array}{lr}\text { 4.3.2 Physical Reference Signals } & 18\end{array}$

4.4 MIMO and Beamforming $\quad 22$

$\begin{array}{lll}4.5 & \text { Summary } & 24\end{array}$

5 Capacity 24

5.1 Introduction 24

5.2 LTE Capacity 24

$\begin{array}{lll}5.3 & \text { NR Capacity } & 27\end{array}$

5.3.1 Parameter Settings 27

$\begin{array}{lll}5.3 .2 & \text { Overheads } & 29\end{array}$

5.3.3 Blind Transmissions $\quad 34$

5.3.4 NR Capacity Results 36

5.4 LTE and NR Capacity Comparison 37

$\begin{array}{lll}5.5 & \text { Summary } & 38\end{array}$

6 Quality of Service $\quad 39$

6.1 Introduction 39

6.2 Overview of New Radio Quality of Service Model 40

6.3 SDAP Layer for Quality of Service $\quad 42$

6.4 Quality of Service over New Radio Sidelink 44 
6.5 Minimum Communication Range 46

6.6 Quality of Service Support for Public Safety 48

6.7 Summary 49

7 LTE/NR Coexistence $\quad 49$

$\begin{array}{lll}7.1 & \text { Introduction } & 49\end{array}$

$\begin{array}{ll}7.2 & \text { DSS Overview }\end{array} 49$

7.3 Sidelink Considerations 53

$\begin{array}{lll}7.4 & \text { Summary } & 54\end{array}$

8 Ongoing Standardization $\quad 54$

$\begin{array}{lll}8.1 & \text { Introduction } & 54\end{array}$

8.2 Proximity based Services in 5GS work items 55

8.2.1 Study Item phase $\quad 55$

$\begin{array}{ll}\text { 8.2.2 Work Item phase } & 58\end{array}$

8.3 NR sidelink enhancement work item 58

8.4 Enhanced V2X services work items 59

$\begin{array}{lll}8.5 & \text { Summary } & 60\end{array}$

9 Conclusion $\quad 61$

$\begin{array}{ll}\text { References } & 62\end{array}$ 


\section{List of Tables}

Table 1 Frame structure parameters and numerologies. $\quad 12$

Table 2 Choices of $N_{\text {period }}^{\text {S-SSB }}$ vs. $\mu$ and frequency range. 21

Table 364 QAM MCS Table $\quad 28$

Table 4 256 QAM MCS Table $\quad 28$

Table 5 NR Channel Bandwidth Table 29

Table 6 Configurations for Band n14 10 MHz NR SL 29

Table 7 Parameter Settings for NR Capacity 29

Table 8 Physical Sidelink Shared Channel (PSSCH) Demodulation Reference Signal (DM-RS) Time-domain Location 33

Table 9 Resource Allocation of Overhead $(\mathrm{OH})$ Items

Table 10 Resource Allocation with Blind Transmissions 36

Table 11 NR SL Capacity in Mbit/s 37

Table 12 NR QoS Mapping Operations 41

Table 13 SL related standardized 5G QoS Identifier (5QI) to QoS characteristics mapping 42

Table 14 Standardized PC5 5QI (PQI) to QoS characteristics mapping 46

Table 15 LTE SL and NR SL channels

\section{List of Figures}

Fig. 1 NR SL resource pool configuration. 9

Fig. 2 Relations of physical resources and frame structure. 14

Fig. 3 A slot format without Physical Sidelink Feedback Channel (PSFCH). Normal cyclic prefix is assumed.

Fig. 4 A slot format with PSFCH. Normal cyclic prefix is assumed.

Fig. 5 Allocation of PSSCH DM-RS, 2nd-stage Sidelink Control Information (SCI) and Sidelink Shared Channel (SL-SCH) modulation symbols.

Fig. 6 Frequency locations of Physical Sidelink Control Channel (PSCCH) DM-RS. 19

Fig. 7 Transmission of Sidelink Synchronization Signal (S-SS)/Physical Sidelink Broadcast Channel (PSBCH) blocks with $\mu=1$.

Fig. 8 A slot format of Sidelink Synchronization Signal Block (S-SSB) with normal cyclic prefix.

Fig. 9 LTE's SL periodic transmission of PSCCH and PSSCH

Fig. 10 LTE SL capacity, without considering overhead from Hybrid Automatic Repeat Request (HARQ), achieved for each period length, with a $2 \mathrm{~ms}$ long PSCCH.

Fig. 11 LTE SL capacity, considering overhead from HARQ, achieved for each period length, considering HARQ blind retransmissions. The PSCCH is $2 \mathrm{~ms}$ long and the PSSCH lasting the remaining period length, respectively.

Fig. 12 A slot structure configuration with minimal $\mathrm{OH}$. 
Fig. 13 Capacity - NR vs LTE. $\quad 38$

Fig. 14 QoS architecture $\quad 40$

Fig. 15 SDAP sublayer structure view 43

Fig. 16 Handling of PC5 QoS Flows based on PC5 QoS Rules 45

Fig. 17 Overview of Minimum Communication Range (MCR) for group communication. $\quad 47$

Fig. 18 Xn setup and Node Configuration messages. 50

Fig. 19 Example of protected resources pattern definitions in Uplink (UL). 51

Fig. 20 Xn resource coordination messages.

Fig. 21 Example use of the Data Traffic Sharing Resource Indication field. 53

Fig. 22 Protocol stack for direct discovery. $\quad 56$ 


\title{
Definitions
}

PC5 A reference point where the User Equipment (UE) directly communicates with another UE over the direct channel.

Uu The Radio interface between UTRAN and the User Equipment.

\author{
Abbreviations \\ 3GPP Third Generation Partnership Project \\ 4G Fourth Generation \\ 5G Fifth Generation \\ 5GC 5G Core Network \\ 5GS 5G System \\ 5QI 5G QoS Identifier
}

ACK Acknowledgement

AGC Automatic Gain Control

AMBR Aggregate Maximum Bit Rate

ARFCN Absolute Radio Frequency Channel Number

ARIB Association of Radio Industries and Businesses

ARP Address Resolution Protocol

ARP Allocation and Retention Priority

AS Access Stratum

ATIS Alliance for Telecommunications Industry Solutions

BWP Bandwidth Part

CA Carrier Aggregation

CCSA China Communications Standards Association

CP Cyclic Prefix 
CQI Channel Quality Indication

CRB Common Resource Block

CRC Cyclic Redundancy Check

CRS Cell Reference Signal

CSI Channel State Information

CSI-RS Channel-State Information Reference Signal

D2D Device-to-Device

DC Direct Current

DL Downlink

DM-RS Demodulation Reference Signal

DRB Data Radio Bearer

DRX Discontinuous Reception

DSS Dynamic Spectrum Sharing

E-UTRA Evolved Universal Terrestrial Radio Access

eMBB enhanced Mobile BroadBand

ETSI European Telecommunications Standards Institute

FDD Frequency Division Multiplexing

FR2 Frequency Range 2

GBR Guaranteed Bit Rate

GFBR Guaranteed Flow Bit Rate

HARQ Hybrid Automatic Repeat Request

IE Information Element

IoT Internet of Things

IPv4 Internet Protocol version 4 
LCID Logical Channel ID

LCP Logical Channel Prioritization

LDPC Low Density Parity Check

LTE Long Term Evolution

MAC Medium Access Control

MBSFN Multicast-Broadcast Single-Frequency Network

MCPTT Mission Critical Push-to-Talk

MCR Minimum Communication Range

MCS Modulation and Coding Scheme

ME Mobile Equipment

MFBR Maximum Flow Bit Rate

MIMO Multiple-Input and Multiple-Output

mMTC massive Machine Type Communication

mmWave Millimeter Wave

MU-MIMO Multi-User MIMO

NACK Negative Acknowledgement

NAS Non-Access Stratum

NB-IoT NarrowBand-Internet of Things

NG-RAN Next-Generation Radio Access Network

NR New Radio

OFDM Orthogonal Frequency-Division Multiplexing

OH Overhead

PAPR Peak-to-Average-Power Ratio

PCF Policy Control Function

PCI Physical Cell ID 
PDB Packet Delay Budget

PDCCH Physical Downlink Control Channel

PDCP Packet Data Convergence Protocol

PDSCH Physical Downlink Shared Channel

PDU Protocol Data Unit

PER Packet Error Rate

PFI PC5 QoS Flow Identifier

PHY Physical Layer

PLMN Public Land Mobile Network

PMI Precoder Matrix Indicator

PPPP ProSe Per-Packet Priority

PPPR ProSe Per-Packet Reliability

PQI PC5 5QI

PRACH Physical Random Access Channel

PRB Physical Resource Block

ProSe Proximity Services

PSBCH Physical Sidelink Broadcast Channel

PSCCH Physical Sidelink Control Channel

PSDCH Physical Sidelink Discovery Channel

PSFCH Physical Sidelink Feedback Channel

PSSCH Physical Sidelink Shared Channel

PT-RS Phase-Tracking Reference Signal

PUCCH Physical Uplink Control Channel

PUSCH Physical Uplink Shared Channel

QAM Quadrature Amplitude Modulation 
QCI QoS Class Identifier

QFI QoS Flow Identifier

QoS Quality of Service

QPSK Quadrature Phase Shift Keying

RAN Radio Access Network

RB Resource Block

RE Resource Element

Rel-12 Release 12

Rel-13 Release 13

Rel-14 Release 14

Rel-15 Release 15

Rel-16 Release 16

Rel-17 Release 17

RF Radio Frequency

RI Rank Indication

RLC Radio Link Control

RQA Reflective QoS Attribute

RRC Radio Resource Control

S-SS Sidelink Synchronization Signal

S-SSB Sidelink Synchronization Signal Block

SC-FDMA Single-Carrier FDMA

SCI Sidelink Control Information

SCS Subcarrier Spacing

SDAP Service Data Application Protocol

SDU Service Data Unit 
SFCI Sidelink Feedback Control Information

SFN System Frame Number

SIB System Information Block

SL Sidelink

SL-BCH Sidelink Broadcast Channel

SL-SCH Sidelink Shared Channel

SPS Semi-Persistent Scheduling

SPSS Sidelink Primary Synchronization Signal

SRB Signaling Radio Bearer

SRS Sounding Reference Signal

SSB Synchronization Signal Block

SSSS Sidelink Secondary Synchronization Signal

SU-MIMO Single-User MIMO

TB Transport Block

TDD Time Division Multiplexing

TRPs Transmitter and Receiver Points

TS Technical Specification

TSDSI Telecommunications Standards Development Society, India

TTA Telecommunications Technology Association

TTC Telecommunications Technology Committee

U2N UE-to-Network

U2U UE-to-UE

UE User Equipment

UL Uplink

UP User Plane 
URLLC Ultra-Reliable and Low Latency Communication

USIM Universal Subscriber Identity Module

V2P Vehicular-to-Pedestrian

V2X Vehicle-to-Everything

V2XP V2X Policy

VRB Virtual Resource Block

VRU Vulnerable Road User 


\section{Executive Summary}

Direct mode communication for first responders over Fourth Generation (4G) Long Term Evolution (LTE) was first introduced by the Third Generation Partnership Project (3GPP) in Release 12 (Rel-12). It is supported by combining the Device-to-Device (D2D) capability, called Sidelink (SL), which enables devices to communicate directly without packets going through the core network, with the off-network Mission Critical Push-to-Talk (MCPTT) voice service. Since then, the cellular technology has evolved to Fifth Generation (5G) New Radio (NR) to provide higher capacity, lower latency, and support for a higher number of devices. While NR Sidelink (SL) was introduced in Release 16 (Rel-16), only enhanced Vehicle-to-Everything (V2X) services have been defined to use it. There is an increasing interest by public safety organizations, such as FirstNet, to leverage NR SL to enable direct mode communications for first responders. In this study, we focus on the specifications available in Rel-16 to understand the current capabilities and limitations of NR SL in the context of direct mode communications for public safety. This document covers the topics of resource management, physical layer specifications, capacity enhancements, Quality of Service (QoS), and coexistence between LTE and NR. We conclude the study by highlighting on-going standardization efforts in Release 17 (Rel-17). In each section, we describe the features or capabilities of NR SL and their applicability to public safety communications.

We start by analyzing the specifications to understand how SL resources are allocated to each application, how to determine the type of communication to use (e.g., unicast, groupcast, or broadcast), and how to provision application service requirements. We describe the mechanisms to authorize and provision resources for NR V2X services based on Rel-16 as it is currently the only service defined to use the SL. Configuration information can be pre-configured inside the devices or dynamically provided by the network through User Equipment (UE) policies. A UE may be authorized to use the NR SL when in outof-coverage, and resources are provided based on geographical areas. When in-coverage, authorization is based on the Public Land Mobile Network (PLMN) ID and resources are provided by the serving cell. Further description of the V2X Policy (V2XP) shows that a V2X service is mapped to a list of authorized NR frequencies (defined by the geographical area), destination IDs to establish communication, Quality of Service (QoS) parameters, and security configuration. We also provide a description of the SL resource allocation, including the SL resource pool, which defines the time and frequency resources that would be used for SL communication. Based on the analysis of the current specifications, we discuss several enhancements needed to support non-V2X applications (e.g., D2D applications), including mechanisms to provision resources for different types of applications and managing concurrent applications with potentially conflicting SL resource allocations.

We further investigate how devices access the resources allocated to SL by focusing on the NR SL Physical Layer (PHY). We first introduce the frame structure and physical resources, then detail the SL channels and reference signals. Finally, we present the Multiple-Input and Multiple-Output (MIMO) and beamforming techniques. The NR SL 
PHY will have an impact on the performance of supported applications. More specifically, the flexible numerology can reduce the Orthogonal Frequency-Division Multiplexing (OFDM) symbol duration and improve communication latency. A configurable number of retransmissions and the Physical Sidelink Feedback Channel (PSFCH), a newly introduced feedback channel at PHY layer, can improve the reliability and spectrum efficiency. In addition, advanced channel coding techniques are used in NR SL, such as Low Density Parity Check (LDPC) for the data channel and polar code for the control channel, to reduce decoding complexity and improve link reliability. We also describe how the Physical Sidelink Control Channel (PSCCH) in NR SL is used to enable channel sensing to avoid collisions and interference. Finally, we highlight the beamforming capability as one of the key techniques to improve the communication range, especially at higher carrier frequencies $(>$ $6 \mathrm{GHz})$.

Leveraging our understanding of the physical layer, we provide our initial investigation on one of the major performance metrics, capacity, in terms of the maximum achievable data rate over SL. The public safety band, Band n14, with $10 \mathrm{MHz}$ bandwidth is selected for numerical analysis, and the focus is on unicast. We study, in detail, NR SL capacity and compare it to the LTE SL capacity. Like NR, NR SL offers flexibility in configurations of the numerology, modulation, and number of retransmissions to adapt to different deployment scenarios and service requirements. For this reason, the study covers various operational configurations and device capacities, together with the associated overheads. Capacity formula with Hybrid Automatic Repeat Request (HARQ) blind transmissions (i.e., no feedback channel) is also derived. The initial quantitative results show that NR capacity varies significantly under different configurations, and the highest is achieved under numerology 0, 256 Quadrature Amplitude Modulation (QAM) capability, and with one blind transmission. Numerology 0 gives higher capacity than numerology 1, and the limit to 64 QAM due to device capability leads to a $1 / 4$ reduction in capacity. In addition, NR capacity is approximately inverse proportional to the number of transmissions when blind transmission is configured. Furthermore, detailed analyses on the impact of individual NR features reveals that the greatest NR capacity improvement over LTE comes from the NR feature of configurable HARQ transmissions, followed by the higher Modulation and Coding Scheme (MCS) (256 QAM and 948/1024 code rate), and then the support of spatial multiplexing. Nevertheless, when NR is configured with a very high number of transmissions - NR allows up to 32 transmissions, including the initial transmission and retransmissions, compared to the fixed four transmissions in LTE - NR could have less capacity. Note that this configuration allows NR to meet the requirement of ultra-high reliability when needed, thus less capacity together with longer latency is balanced out. This work on capacity will be extended to multicast and broadcast communications, consider other capacity measures, such as number of users, and correlate the results with the communication range to obtain an adequate picture of the coverage performance.

We also study the NR QoS model in general, and then take a closer look at the SL related enhancements. QoS is expected to play a significant role when capacity is limited and traffic has to be prioritized. Compared with the relatively simplified QoS operations over 
LTE SL, NR SL's QoS handling has been improved considerably and closely resembles the QoS mechanism for the 5G system communication over NR's Uu interface. To name a few, the application layer data is mapped to QoS flows, and the QoS flows are mapped to the Data Radio Bearers (DRBs) by the Service Data Application Protocol (SDAP) layer, which is a new layer introduced by NR. Applications' QoS requirements can be conveyed through QoS parameters and QoS characteristics of the corresponding QoS flow. A new QoS parameter, Range, may be used to specify the area where the QoS needs to be maintained. In addition, the new NR SL QoS parameters are backward compatible with QoS parameters of LTE Proximity Services (ProSe). With these enhancements as well as the ongoing 3GPP standardization efforts on public safety applications, we are confident that direct mode communications for public safety services can be supported by NR from the QoS perspective.

While NR SL looks promising, NR deployments have just started and LTE will remain the dominant technology for some time, possibly on the same band. This is why we also investigate Dynamic Spectrum Sharing (DSS), a set of features that allows LTE and NR base stations to coordinate the sharing of the same spectrum. This is accomplished by exchanging configuration information, such as Downlink (DL) and Uplink (UL) control regions, and data resource allocation. However, since DSS does not specifically address SL communication, there are some limitations into the current specifications, such as how to signal SL configuration and resource pool allocations. We discuss possible ways to address current limitations depending on the type of communication, though further work is needed to identify the modifications necessary for 3GPP specifications.

Finally, we provide an overview of the study items and work items related to the NR SL that are currently under analysis and specification in 3GPP for the next release (Release 17). The new features include the definition of NR ProSe, comprising direct discovery, direct communication, and UE-based relays. It also provides enhancements to the NR SL power efficiency, reliability, and latency for all services that will use it, including enhanced NR V2X services and NR ProSe services. It is expected that some of those items will respond, at least partially, to limitations identified in this study. However, as shown in this study, additional performance evaluation is needed to understand how to efficiently make use of NR SL for direct mode communications. 


\section{Introduction}

The Third Generation Partnership Project (3GPP) is responsible for developing standards to support the deployment of cellular communications technologies. Multiple releases over the past decade have brought about the widespread deployment of Long Term Evolution (LTE) cellular networks. These " $4 \mathrm{G}$ " systems have enabled their end users to communicate via telephony, text, video, and data transfers.

A feature of 4G LTE cellular systems is their support for direct communication from one User Equipment (UE) to another UE without the transmitted data traversing the base station. Directly-exchanged data and signaling traffic between UEs uses a dedicated set of time and frequency resources known as the "Sidelink (SL)," which 3GPP first introduced in Release 12 (Rel-12) of its mobile communication standards. In LTE, Device-to-Device (D2D) communication over the SL can take place between UEs that are within a base station's coverage area; if the UEs are being used by public safety personnel, they can also use D2D communications while outside of any base station's coverage area.

There are two basic modes of operation for D2D communication via SL: in the first one, UEs send signaling messages to the base station that request grants of time and frequency resources that they will use to exchange data, and the base station is responsible for granting those resources so that multiple UEs can use the SL to communicate without their transmissions' interfering with each other. This mode only applies to in-coverage cases since the UEs need to communicate with the base station. In the second mode of operations, UEs communicate without grants by using advertised or pre-configured SL configuration settings and by selecting time and frequency resources randomly for signaling and data transmissions, possibly introducing UE-to-UE interference due to message collisions. This mode can apply to in-coverage cases but is the only option for out-of-coverage cases.

The original version of ProSe defined three basic functions: Discovery, Synchronization, and Communications. There was no provision for the normal Hybrid Automatic Repeat Request (HARQ) feedback, and the communications function was not integrated with the discovery function, nor was it coupled to the synchronization function. 3GPP continued to add features to ProSe in subsequent releases. Release 13 (Rel-13) defined UE-to-Network (U2N) relays, and Release 14 (Rel-14) extended D2D to encompass many more use cases. These enhancements allow full support for in-coverage, out-of-coverage, and partial coverage scenarios, enabling first responders to communicate all the time. Rel14 also adds support for Vehicle-to-Everything (V2X) communications, albeit via modes of communication separate from D2D communication. Among other functions that use broadcast signaling, Rel-14 added the channel sensing and Semi-Persistent Scheduling (SPS) features, which are designed to reduce the probability of collisions when UEs are operating in out-of-coverage mode. Release 15 (Rel-15) added more features, including Carrier Aggregation (CA), transmission diversity, and support for QAM with a 64-symbol constellation, which allows 6 bits of information to be sent using a single symbol.

The set of releases up to and including Rel-15 are based on the LTE-A interface. The limitations of this interface may prevent future cellular systems from meeting the IMT- 
2020 performance requirements. Thus, during the past several years, 3GPP has begun work on standards for $5 \mathrm{G}$ cellular communications that are based on a new radio interface. These standards include definitions for a new set of radio access technologies collectively known as 5G New Radio (NR). NR operates in two distinct frequency bands: FR1, which is from $410 \mathrm{MHz}$ to $7125 \mathrm{MHz}$, and FR2, which is a set of millimeter wave bands between $24.250 \mathrm{GHz}$ and $52.600 \mathrm{GHz}$ [1, Clause 5.1]. NR is enhancing three key capabilities: The first one, called enhanced Mobile BroadBand (eMBB), is designed to provide higher capacity and very high peak data rates, with expected data rates up to $20 \mathrm{Gbit} / \mathrm{s}$ in the downlink and $10 \mathrm{Gbit} / \mathrm{s}$ in the uplink, and also improved cell edge data rates. This capability aims at supporting applications such as 3D videos and ultra high definition video. The second capability is Ultra-Reliable and Low Latency Communication (URLLC) designed for critical applications such as self-driving (i.e., autonomous) cars, and targets radio access network latency below $1 \mathrm{~ms}$, which is not possible in LTE since the subframe duration is $1 \mathrm{~ms}$. The third capability is massive Machine Type Communication (mMTC) that falls into the smart city concept where there are many sensors providing data, with up to one million devices per square kilometer.

Public safety agencies are interested in how NR can give them the performance improvements that will allow their personnel to remain connected and maintain high levels of situational awareness, even in dangerous environments in which the commercial communications infrastructure is degraded or destroyed. The higher bandwidth and lower latency promised by NR standards have the potential to enable enhanced Mission Critical Push-toTalk (MCPTT) services, as well as new Internet of Things (IoT) related systems for public safety use, such as unmanned vehicles with haptic interfaces, which will enable remote inspection of dangerous environments, such as fires involving hazardous materials. Such systems can also enable virtual or augmented reality applications to support emergency field operations like telemedicine.

3GPP's work includes developing standards for Vehicle-to-Everything (V2X) communications, and the new SL standards that are part of V2X will offer opportunities for new types of public safety applications that will improve interoperability and result in a better user experience. These standards introduce channel sensing and UE feedback, which promise to reduce the impact of collisions and interference. However, this additional complexity can introduce overhead that can decrease capacity [2]. Thus, network operators and equipment manufacturers must use care in designing their systems in order to optimize performance. There are numerous dimensions in the performance metric space that industry must consider, such as capacity and quality of service. The purpose of this document is to examine these major performance metrics and discuss how NR addresses them differently than LTE does, and to identify some of the design tradeoffs that manufacturers and operators will face as they build and deploy NR networks for public safety applications.

This document is organized as follows. In Section 3, we discuss resource management for the SL. We describe the mechanisms to allocate resources for V2X services, and discuss the SL's pool of time-frequency resources. In Section 4, we examine the SL PHY, with an emphasis on the new features that $3 \mathrm{GPP}$ has introduced to support NR, such as multiple 
numerologies and support for antenna beamforming. The NR PHY layer will allow increases in channel capacity, and we perform a detailed analysis in Section 5 to quantify NR's capacity gain relative to LTE, and we examine the effect of various system parameters on capacity, such as the number of retransmissions. In Section 6, we consider the new mechanisms that 3GPP has introduced in NR to support Quality of Service (QoS), and we compare these to the legacy mechanisms in LTE. We then visit the issue of spectrum coexistence between LTE and NR in Section 7; because spectrum management is limited to the Uplink (UL) and Downlink (DL), we summarize the main differences between LTE SL and NR SL and introduce some possible means of supporting coexistence in the SL. Next, we review ongoing standardization work in 3GPP in Section 8, which at the time of this writing is working on Rel-17, and we focus on study items and work items that affect the NR SL. Finally, we summarize the work in this study in Section 9.

\section{Resource Management}

\subsection{Introduction}

Applications and services using direct communication between devices need to be mapped to SL configurations and resources in order to operate properly and obtain the desired performance. In this section, we use the specifications for provisioning V2X services in Rel-16 as our baseline since it is the only type of services that is clearly defined to use SL over the 5G NR air interface. In particular, we focus on the mechanisms in place to allocate resources to a given service, specify QoS parameters, and control the type of communication to be used by a service. We then introduce the NR SL resource pool that specifies the time and frequency resources available for the UEs to communicate over NR SL. Finally, we discuss limitations and possible extensions to support non-V2X applications.

\subsection{V2X Services Provisioning}

The V2X configuration parameters can be pre-configured in the Mobile Equipment (ME) (i.e., UE), configured in the Universal Subscriber Identity Module (USIM), provided by UE policies or a V2X application server, or a combination of those [3]. Priority is given to dynamic information with the highest precedent going to the configuration parameters provided by UE policies [4]. The V2X policies are provided by the Policy Control Function (PCF) as part of the UE policy delivery procedure upon request by a UE connected to the network. The policies are provided in a V2XP UE policy part inside the message sent by the PCF. While V2XP defines policies for V2X communication over both the Uu interface (i.e., with the network) and PC5 interface (i.e., the new D2D interface also known as SL), we only focus on policies related to the PC5 interface. A UE requests updated V2X policies when the validity timer associated with previous policies expires or when there is no information in the UE (e.g., going into a new geographical area or a different network). 


\subsubsection{Authorization}

Authorization to use V2X services is based on whether a UE is connected to a network or not. Inside the V2XP Info message, there is a single parameter to authorize out-of-coverage operations, which is defined by the UE not being served by LTE Evolved Universal Terrestrial Radio Access (E-UTRA) and not being served by 5G NR. This parameter specifies if a UE can perform V2X communication over E-UTRA-PC5 and/or NR-PC5. However, as we will discuss in Section 3.2.3, the resources that can be used will depend on the geographical area where the UE is located. When the UE is in network coverage, authorization to perform V2X is specified per PLMN ID, with specific radio resources provided by the serving cell when the UE connects to the network.

\subsubsection{Service Configuration}

The list of V2X services that can be used, along with their configuration is also included in the V2XP. Each service is uniquely identified by a binary code [5]. There are separate lists for E-UTRA and NR V2X communication, thus allowing the same service to potentially be using either technology.

With regards to NR, a service is mapped to the following parameters:

- NR Frequency to use in given geographical area: configuration allows to specify a list of frequencies that can be used within a list of geographical areas. Each frequency is coded according to the NR-Absolute Radio Frequency Channel Number (ARFCN) value defined in [6], and limited to bands n38 and n47 in Rel-16.

- Destination L2 ID for broadcast: specifies the L2 ID to be used by lower layers for transmitting packets in broadcast communications.

- Destination L2 ID for groupcast: specifies the L2 ID to be used by lower layers for transmitting packets in groupcast communications.

- Destination L2 ID for unicast initial signaling: specifies the L2 ID to be used by lower layers for transmitting packets during initial connection. During unicast connection establishment, the UEs will exchange the L2 IDs to use for data communication.

- PC5 QoS parameters: includes information such as guaranteed and maximum flow bit rate, and range (see Section 6.4 for more details).

- Unicast security policy: indicates cyphering and integrity protection for both signaling and user plane.

- Default communication mode: specifies the default mode of communication (e.g., unicast, broadcast, or groupcast) for this service. 
Not all parameters are configured for all services. For example, if a service only supports broadcast mode, it will not be included in the mapping rules for groupcast or unicast, nor will it have an entry in the unicast security policies. Similarly, mapping rules can be used to map multiple services to the same set of configuration parameters.

Based on that information, when a V2X service starts, its service code will be checked against the list of services that were (pre-)configured. The UE will use the list of NR frequencies configured for that service to see if it can operate in any of those frequencies in its current location. If the UE is out of coverage, this will be done by checking the pre-configured resources. Otherwise, the UE will check if any of the authorized PLMNs is operating in the right frequency and make a selection. If a frequency is available, the service can start and information about destination L2 ID, QoS, and security can be passed to the lower layers to activate SL communication.

\subsubsection{Resource Provisioning}

Similar to what was done with LTE SL, there are different mechanisms for providing NR SL resources to the UEs based on the situation and operator preference. For an out-ofcoverage UE, the resources must be pre-configured in the device. To accommodate the fact that the same set of resources may not be available everywhere, the resources are location dependent. The UE policies associate a geographical area defined by a set of latitude/longitude coordinates to a set of radio resources that can be used. For in-coverage cases, an operator can use the System Information Block (SIB) 12 to broadcast common resource pool information to all UEs. This message also includes special resource pools when the UE experiences radio link failure or handover. Alternatively, an operator can decide to use dedicated resource pools for each UE and transmit the resource configuration via Radio Resource Control (RRC) signaling (i.e., in an RRCReconfiguration message).

We note that even though the Information Elements (IEs) defined in TS 38.331 [7] contain a list of configurations for multiple frequency carriers, Rel-16 indicates that only one element can be configured in the list. That is, in Rel-16, multiple applications will share the same frequency band and SL Bandwidth Part (BWP), but they could use different resource pools.

\subsection{Sidelink Resource Allocation}

The configuration of the SL BWP and resource pools is established by the RRC layer [7] and provided to lower layers when activated. In Rel-16, UEs can only have one active SL BWP at a time in a given frequency band. Just like a regular BWP, a SL BWP is defined by its frequency, bandwidth, Subcarrier Spacing (SCS), and Cyclic Prefix (CP). In addition, it also defines parameters common to all the resource pools that are contained within it, namely the number of symbols and starting symbol used for SL in all slots (except those with Synchronization Signal Block (SSB)), power control for Physical Sidelink Broadcast Channel (PSBCH), and optionally the location of the Direct Current (DC) subcarrier. 
The SL BWP has different lists of resource pools for transmissions and receptions, to allow for a UE to transmit in a pool and receive in another one. For transmissions, there is one pool for UE selected mode, one for scheduled mode (e.g., when the gNodeB helps with resource selection), and one for exceptional situations. Resource pools are expected to be used for only transmission or reception, except when the feedback mechanisms are activated, in which case a UE would transmit Acknowledgement (ACK) messages in a reception pool and receive ACK messages in a transmission pool.

As shown in Fig. 1, a resource pool located inside an SL BWP is defined by a set of $s l$-Rb-Number contiguous Resource Blocks (RBs) in the frequency domain starting at $\mathrm{RB}$ sl-StartRBsubchannel. The resource pool is further divided into subchannels of size sl-SubchannelSize, which can take one of several values (i.e., 10, 12, 15, 20, 25, 50, 75, and 100). The notion of a subchannel is essential for the sensing mechanisms designed by 3GPP. Depending on the value of sl-RB-Number and sl-SubchannelSize, some RBs inside the resource pool may not be used by the UEs. As such, some consistency is needed in order to avoid wasting resources.

In the time domain, a resource pool has some available slots configured by various parameters. To determine which slots belong to the pool, a series of criteria are applied: 1) Slots where SSB will be transmitted cannot be used. The number and locations of those slots are based on configuration, as detailed in Section 4.3. 2) Slots that are not allocated for UL (e.g., in the case of Time Division Multiplexing (TDD)) or do not have all the symbols available (as per SL BWP configuration) are also excluded from the resource pool. 3) Some slots are reserved such that the number of remaining slots is a multiple of the sl-TimeResource-r16 bitmap length (also defined as $L_{\text {bitmap }}$ ), that can range from 10 bits to 160 bits. The reserved slots are spread throughout $10240 * 2^{\mu}$ slots, where $\mu$ is the numerology. 4) The bitmap sl-TimeResource-r16 is applied to the remaining slots to compute the final set of slots that belong to the pool, which are labeled $t^{\prime S L}{ }_{i}$, with i between 0 and $T_{\text {max }}^{\prime}$, and repeats every $10240 * 2^{\mu}$ slots.

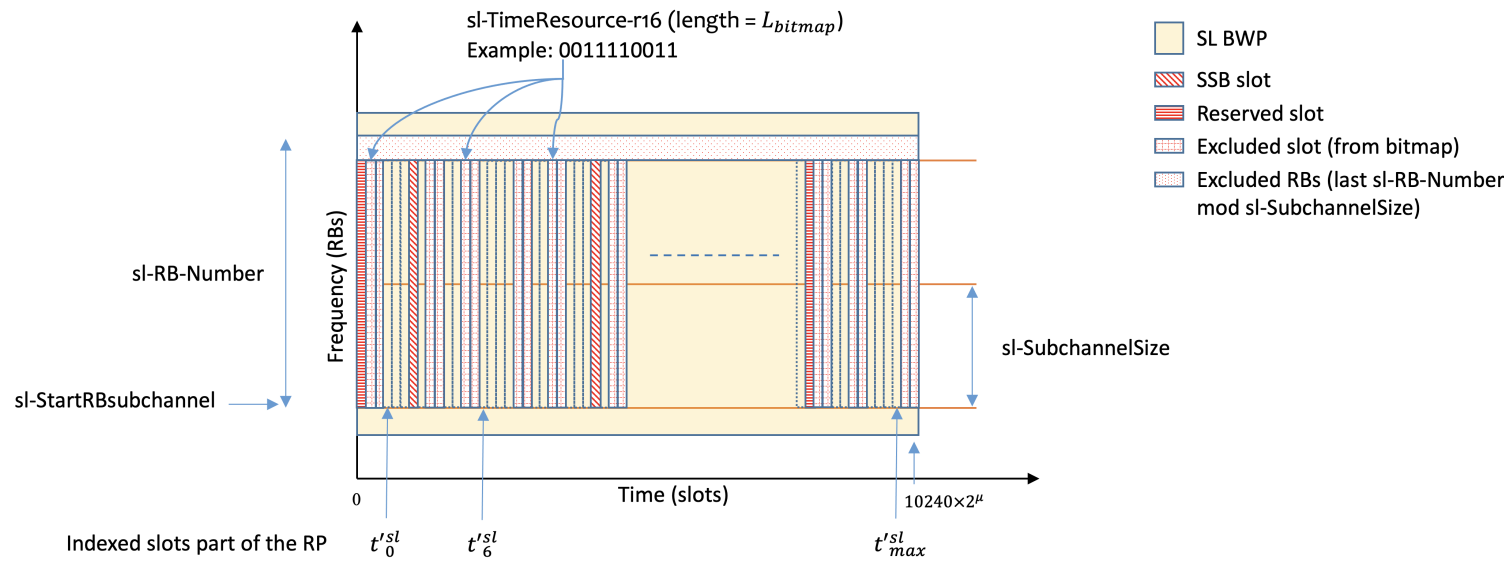

Fig. 1. NR SL resource pool configuration.

Transmissions within the resource pools are based on one of two modes: in Mode 1, the 
resources are allocated by the gNodeB and it supports dynamic and periodic allocations, while in Mode 2, UEs autonomously select resources based on sensing. A description of those two modes can be found in [8, Section 6.3.2] while Section 4 provides details on the SL channels transmitted within the resource pools.

\subsection{Non-V2X applications}

From an Access Stratum (AS) point of view, the SL can be used by any type of applications. Unlike LTE SL, which defined different modes for D2D and V2X, there are only 2 modes of communication depending on whether the resource selection is assisted by the network or independently done by the UEs. As long as the performance provided by NR SL meets public safety requirements, it is likely that changes required in the AS will be minimal. However, though mechanisms for provisioning non-V2X applications will be very similar to those for V2X services, new specifications will be required. Specifically, a new addressing scheme to identify applications will be needed.

Another issue with supporting non-V2X applications is the handling of concurrent applications. It is more likely that non-V2X applications and V2X services will need to operate on different bands. For example, Direct Mode for MCPTT may be configured to use a regular cellular band (e.g., n2, n4, n5, n12, n13) or a public safety band (e.g., n14 in the US), while V2X services are configured with any operating bands defined for V2X (i.e., n38 or n47 in Rel-16 [6]). This could be the case if a fire truck acts as a U2N Relay and is equipped with V2X services for collision avoidance and other safety services. Similarly, a public safety handheld device could also have Direct Mode and Vehicular-to-Pedestrian (V2P) services running. Supporting those use cases will require further evaluation in order to handle device limitations, such as operating on multiple bands simultaneously (or in an alternating fashion) or prioritizing traffic.

\subsection{Summary}

In Rel-16, 3GPP has defined mechanisms to enable and configure V2X services over SL. Resources can be allocated based on the device location and the network it is connected to. Similar mechanisms are required for non-V2X applications and operators will need to ensure consistency in the UE policies so the devices can communicate with each other. Concurrent applications between V2X services and non-V2X applications will need further study to ensure proper operations, especially due to the current restrictions in the number of SL BWPs and resource pools that can be configured at a given time. Finally, while NR provides a lot of flexibility to configure the resource pools, analysis of the SL performance considering public safety traffic and scenarios is needed to guide the allocation policies. 


\section{Physical Layer}

\subsection{Introduction}

Like any wireless communication system, the PHY is an important layer in NR. Various new PHY features have been introduced in NR to support different emerging applications, which demand high throughput, high reliability, and low latency, among others. In this section, we introduce some critical SL PHY features, channels, reference signals, and MIMO and beamforming, all of which having an impact on user coverage. In particular, Section 4.2 covers configurable numerology, flexible time-domain frame structure, and frequency domain resource structure. Section 4.3 introduces the PHY channels and PHY reference signals defined for NR SL. Section 4.4 presents the MIMO and beamforming development in NR standardization. Finally, Section 4.5 summarizes the key PHY features introduced in NR.

\subsection{Frame Structure and Physical Resources}

\subsubsection{Numerologies, Frames, Subframes, and Slots}

In terms of frame structure, NR SL is similar to LTE in that 1) the duration of each frame and subframe are $10 \mathrm{~ms}$ and $1 \mathrm{~ms}$, respectively [9, Section 4.3.1], and 2) the number of consecutive Resource Elements (REs)/subcarriers per RB, $N_{\mathrm{sc}}^{\mathrm{RB}}$, is 12 [9, Section 4.4.4].

NR SL is advantageous over LTE SL in the reconfigurability in both frequency and time domains. One contributing factor is the numerology $\mu$, which defines SCS, number of slots in a subframe, and cyclic prefix options. In NR SL, the value of $\mu$ ranges from 0 to 3 [9, Section 8.2.2], whereas in NR UL and DL, 4 is also supported [9, Section 4.2]. In addition, the supported $\mu$ values vary at different frequency bands.

In the frequency domain, SCS is defined by $\mu$ as [9, Section 4.4.4]

$$
\Delta f=2^{\mu} \cdot 15[\mathrm{kHz}] \text {. }
$$

Two types of cyclic prefix are defined: normal and extended cyclic prefix. Normal cyclic prefix is supported for all $\mu$, whereas only $\mu=2$ supports extended cyclic prefix [9, Table 4.2-1]. When normal cyclic prefix is applied, each slot contains 14 OFDM symbols [9, Table 4.3.2-1]. The number of OFDM symbols is 12 for extended cyclic prefix [9, Table 4.3.2-2].

Within a subframe, slots are numbered from 0 to $N_{\text {slot }}^{\text {subframe, } \mu}-1$, where $N_{\text {slot }}^{\text {subframe, } \mu}$ is the number of slots per subframe for $\mu$, in the form of

$$
N_{\text {slot }}^{\text {subframe }, \mu}=2^{\mu}
$$

and the duration of a slot is

$$
\tau_{\text {slot }, \mu}=\frac{1}{2^{\mu}}[\mathrm{ms}]
$$


To sum it up, parameters and numerologies for frame structure are defined by $\mu$ as in Table 1 , where $N_{\text {symb }}^{\text {slot }}$ is the number of symbols per slot, $N_{\text {slot }}^{\text {frame } \mu}$ is the number of slots per frame for $\mu$, and $N_{\text {symb }}^{\text {subframe, } \mu}$ is the number of symbols per subframe for $\mu$.

Table 1. Frame structure parameters and numerologies.

\begin{tabular}{|c|c|c|c|c|c|c|c|}
\hline$\mu$ & $\mathrm{SCS}[\mathrm{kHz}]$ & $N_{\text {slot }}^{\text {frame }, \mu}$ & $N_{\text {slot }}^{\text {subframe }, \mu}$ & $\tau_{\text {slot }, \mu}[\mathrm{ms}]$ & $\begin{array}{c}\text { Cyclic } \\
\text { Prefix }\end{array}$ & $N_{\text {symb }}^{\text {slot }}$ & $N_{\text {symb }}^{\text {subframe }, \mu}$ \\
\hline 0 & 15 & 10 & 1 & 1 & Normal & 14 & 14 \\
\hline 1 & 30 & 20 & 2 & 0.5 & Normal & 14 & 28 \\
\hline 2 & 60 & 40 & 4 & 0.25 & Normal & 14 & 56 \\
\cline { 5 - 8 } & 120 & 80 & 8 & 0.125 & Extended & 12 & 48 \\
\hline 3 & 12 & & Normal & 14 & 112 \\
\hline
\end{tabular}

\subsubsection{Physical Resources}

Here we introduce several terms commonly used in the NR physical resource description.

Resource Grid: The resources for NR SL can be represented by a resource grid. Each of the UL, DL, and SL channels are configured with its set of resource grids, and for SL, it is composed of $N_{\text {grid, } \mathrm{SL}}^{\mathrm{size}}, N_{\mathrm{sc}}^{\mathrm{RB}}$ subcarriers in the frequency domain and $N_{\text {symb }}^{\text {subframe }, \mu}$ OFDM symbols in the time domain, where $N_{\text {grid, SL }}^{\text {size }, \mu}$ is the size of the resource grid for $\mu$ that indicates the number of RBs in the frequency domain, and is related to carrier bandwidth. The resource grid starts from $n_{\mathrm{CRB}}^{\mu}=N_{\text {grid }}^{\text {start }, \mu}$, where $n_{\mathrm{CRB}}^{\mu}$ is the index of Common Resource Block (CRB) and $N_{\text {grid }}^{\text {start } \mu}$ is configured at the RRC layer [7, Section 6.3.5].

Resource Element: The unit element in a resource grid is RE, which occupies one subcarrier in the frequency domain and one OFDM symbol in the time domain. REs can be indexed as $(k, l)_{p, \mu}$, where $k$ is the index in the frequency domain, $l$ is the symbol position in the time domain, and $p$ is the antenna port. RE with $k=0$ coincides with the subcarrier at Point A, which, for NR SL, is the frequency-location expressed as in ARFCN [9, Section 4.4.4.2], [9, Section 8.2.7].

Resource Block and Common Resource Block: In the frequency domain, the general concept of an RB contains 12 consecutive subcarriers, as mentioned above. On the other hand, $\mathrm{CRB}$ is the indexed RB with its index $n_{\mathrm{CRB}}^{\mu}$ incremented from $0 . n_{\mathrm{CRB}}^{\mu}$ is related to the frequency index of RE as follows [9, Section 4.4.4.4]:

$$
n_{\mathrm{CRB}}^{\mu}=\left\lfloor\frac{k}{N_{\mathrm{sc}}^{\mathrm{RB}}}\right\rfloor .
$$

Bandwidth Part and Physical Resource Block: Unlike LTE, 5G NR introduces BWP, which provides more flexibility in frequency resource configuration. For a given numerology $\mu_{i}$, a BWP $i$ is a subset of contiguous CRBs. Only one BWP can be configured for NR 
SL [10, Section 16], whereas for each of the NR UL and DL channels, up to four BWPs can be configured and only one can be active [9, Section 4.4.5].

The BWP starts at $n_{\mathrm{CRB}}^{\mu}=N_{\mathrm{BWP}, i}^{\mathrm{start}, \mu}$, and it contains $N_{\mathrm{BWP}, i}^{\mathrm{size}, \mu} \mathrm{RBs}$. It is bound by the resource grid as [9, Section 4.4.5]

$$
N_{\text {grid, SL }}^{\text {start } \mu} \leq N_{\mathrm{BWP}, i}^{\mathrm{start}, \mu}<N_{\text {grid, SL }}^{\mathrm{start}, \mu}+N_{\text {grid, SL }}^{\mathrm{size}, \mu}
$$

and

$$
N_{\text {grid, SL }}^{\text {start } \mu}<N_{\mathrm{BWP}, i}^{\mathrm{start}, \mu}+N_{\mathrm{BWP}, i}^{\mathrm{size}, \mu} \leq N_{\mathrm{grid}, \mathrm{SL}}^{\mathrm{start}, \mu}+N_{\text {grid, SL }}^{\text {size } \mu}
$$

RBs within a BWP are called Physical Resource Blocks (PRBs) and are numbered from 0 to $N_{\mathrm{BWP}, i}^{\mathrm{start}, \mu}-1$. PRB indexes are related to CRB indexes as [9, Section 4.4.4.4]

$$
n_{\mathrm{CRB}}^{\mu}=n_{\mathrm{PRB}}^{\mu}+N_{\mathrm{BWP}, i}^{\mathrm{start}, \mu}
$$

In NR, since each device may operate in a different BWP and using a different numerology, the common resource block is necessary for a device to locate the frequency resources within the carrier bandwidth. Fig. 2 summarizes the relations of physical resources and frame structure as described above, including the resource grid, CRB, RE, BWP, PRB, slot, and subframe. Their sizes and boundaries of indexes are also specified, in both the time and frequency domains.

\subsection{Sidelink Channels and Reference Signals}

The PHY of NR SL carries the physical channels of

- Physical Sidelink Shared Channel (PSSCH),

- Physical Sidelink Control Channel (PSCCH),

- Physical Sidelink Broadcast Channel (PSBCH), and

- Physical Sidelink Feedback Channel (PSFCH),

reference signals of

- Demodulation Reference Signal (DM-RS) for Physical Sidelink Shared Channel (PSSCH), $\mathrm{PSCCH}$, and PSBCH,

- Phase-Tracking Reference Signal (PT-RS), and

- Channel-State Information Reference Signal (CSI-RS), and Sidelink Synchronization Signal (S-SS) of

- Sidelink Primary Synchronization Signal (SPSS), and

- Sidelink Secondary Synchronization Signal (SSSS). 


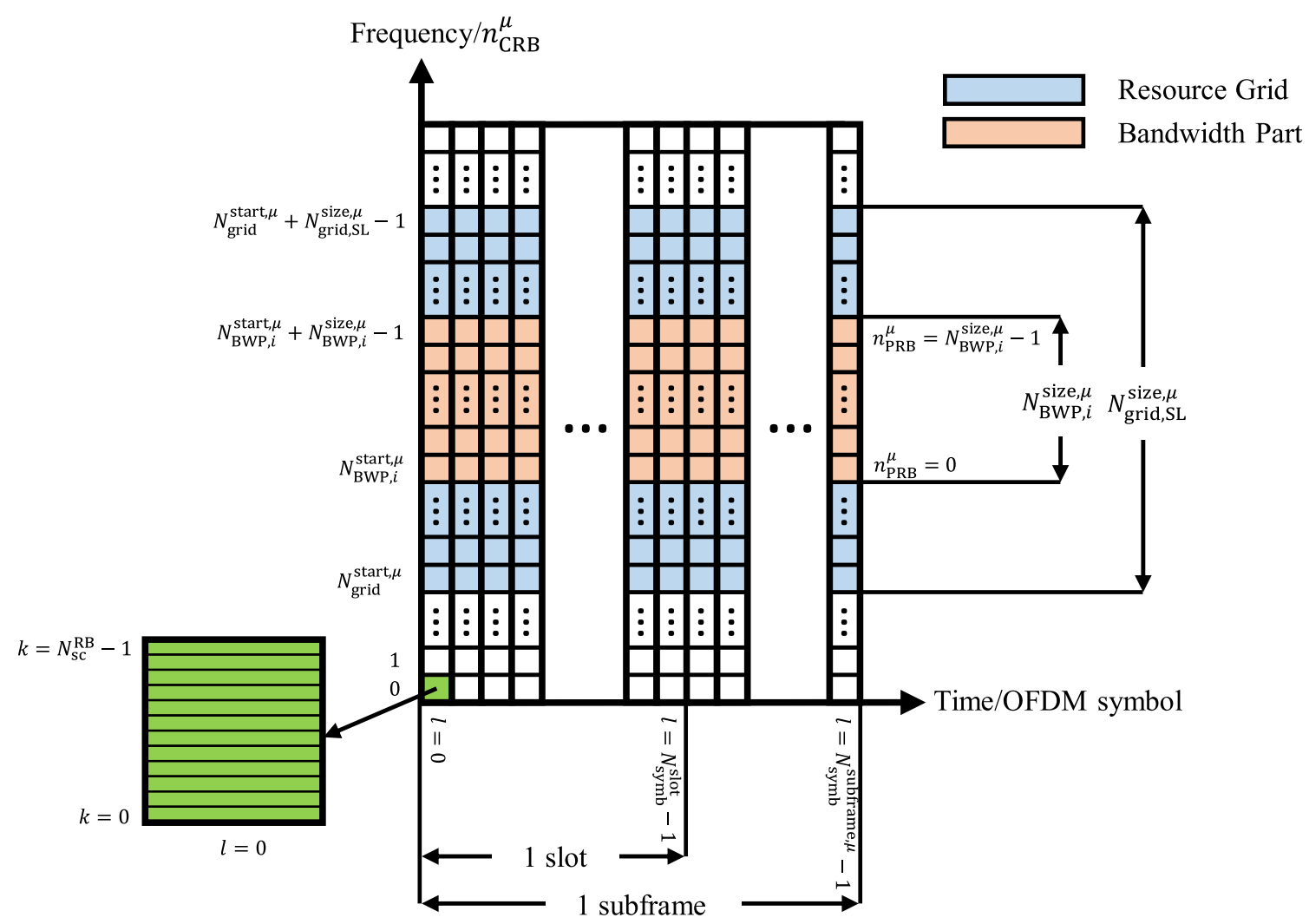

Fig. 2. Relations of physical resources and frame structure.

In addition, a series of consecutive OFDM symbols of Sidelink Primary Synchronization Signal (SPSS), Sidelink Secondary Synchronization Signal (SSSS) and PSBCH form a Sidelink Synchronization Signal (S-SS)/PSBCH block, or S-SSB.

These physical channels, physical signals and the S-SS/PSBCH block are discussed in this section.

As discussed in Section 4.2, a slot contains 14 OFDM symbols with a normal cyclic prefix and 12 symbols with an extended cyclic prefix. The physical channels of PSCCH, PSSCH and/or PSFCH, as well as the reference signals, fit into the same slot. Fig. 3 illustrates a slot format that contains PSCCH and PSSCH, with the PSFCH disabled. When the PSFCH is requested, three slots that were supposed to be allocated to the PSSCH are reassigned to the PSFCH, as well as its Automatic Gain Control (AGC) and guard symbols. An example of such a slot format is shown in Fig. 4. Examples in both Fig. 3 and Fig. 4 assume that a normal cyclic prefix is applied. [8, Section 6.2.1]

\subsubsection{Physical Sidelink Channels}

As listed in [11, Table 4.3-1] and [11, Table 4.3-2], SL transport channels are mapped to physical channels as: 


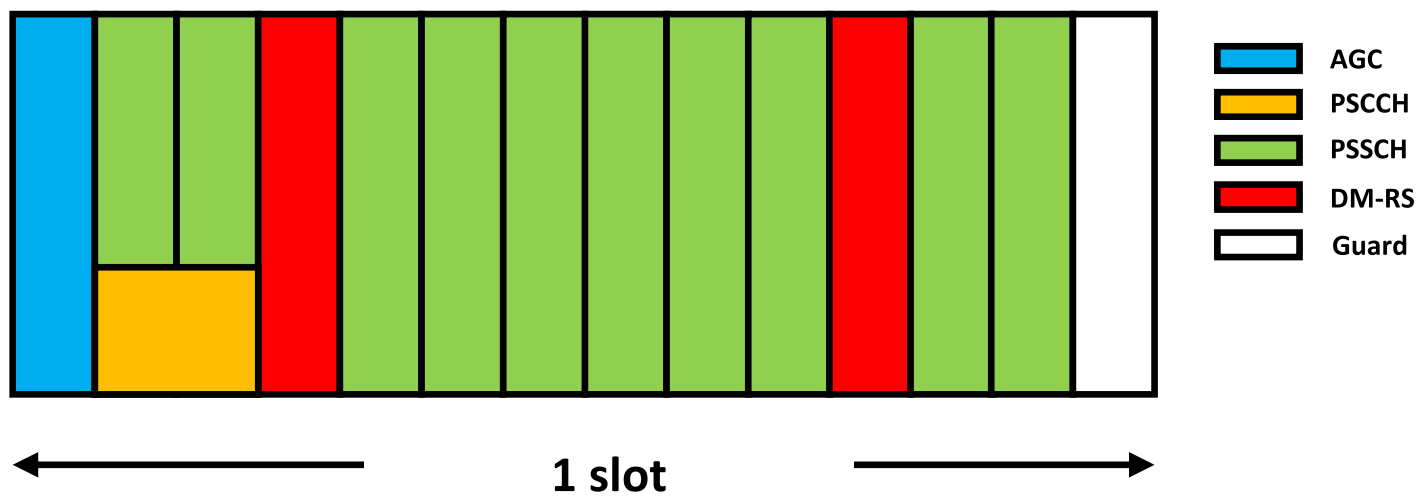

Fig. 3. A slot format without PSFCH. Normal cyclic prefix is assumed.

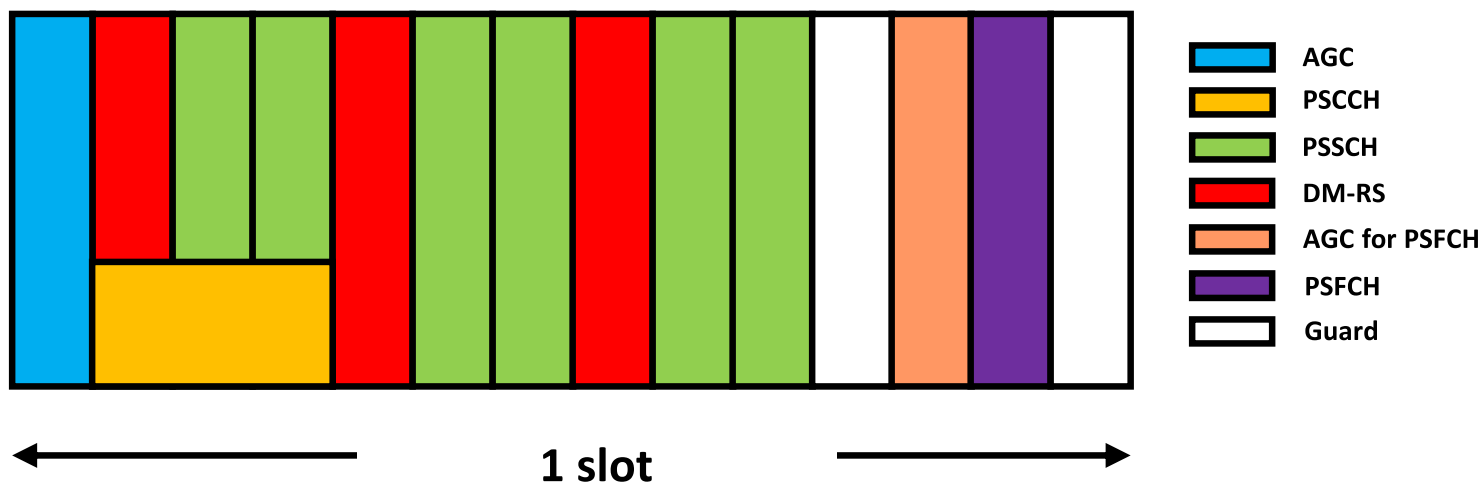

Fig. 4. A slot format with PSFCH. Normal cyclic prefix is assumed.
- $\mathrm{SL}-\mathrm{SCH} \quad \Longrightarrow \quad \mathrm{PSSCH}$,
- $\mathrm{SL}-\mathrm{BCH} \quad \Longrightarrow \quad \mathrm{PSBCH}$,

and SL control information and SL feedback control information are mapped to physical channels as:

- 1st-stage SCI $\Longrightarrow$ PSCCH,

- 2nd-stage SCI $\Longrightarrow \mathrm{PSSCH}$,

- SFCI $\quad \Longrightarrow \quad$ PSFCH.

PSSCH:

PSSCH carries Sidelink Shared Channel (SL-SCH) Transport Block (TB) from the transport layer, as well as the 2nd-stage Sidelink Control Information (SCI). At the transport layer, the SL-SCH data and 2nd-stage SCI are attached with Cyclic Redundancy Check (CRC), channel coded, rate matched and then multiplexed. Channel coding for SL-SCH is LDPC, whereas the 2nd-stage SCI is Polar coded. 
At the PHY, the block of bits are first scrambled and then modulated. The modulation for the 2nd-stage SCI bits is performed using Quadrature Phase Shift Keying (QPSK), resulting in $M_{\mathrm{bit}, \mathrm{SCI} 2}^{(q)} / 2$ modulation symbols, where $M_{\mathrm{bit}, \mathrm{SCI} 2}^{(q)}$ is the number of coded bits for the 2nd-stage SCI, and 2 is the QPSK modulation order. Modulation schemes for SLSCH bits include QPSK, 16 QAM, 64 QAM, and 256 QAM, and their modulation orders $Q_{m}$ are 2, 4, 6, and 8, respectively. Using one of the modulation schemes, the number of modulation symbols is $M_{\mathrm{bit}, \text { data }}^{(q)} / Q_{m}$, where $M_{\mathrm{bit} \text {, data }}^{(q)}$ is the number of coded bits for the SL-SCH. [9, Section 8.3.1.2]

The modulation symbols $d^{(q)}(0), \ldots, d^{(q)}\left(M_{\mathrm{symb}}^{(q)}-1\right)$ can be mapped up to $v=2$ layers [9, Section 8.3.1.3]:

$$
\begin{aligned}
x(i) & =\left[\begin{array}{lll}
x^{(0)}(i) & \ldots & x^{(v-1)}(i)
\end{array}\right]^{\mathrm{T}}, \\
i & =0,1, \ldots, M_{\mathrm{symb}}^{\text {layer }}-1, \\
v & \in\{1,2\},
\end{aligned}
$$

where $M_{\mathrm{symb}}^{(q)}$ is the combined number of modulation symbols for the 2nd-stage SCI and SL-SCH. When $v=1$ [9, Table 7.3.1.3-1],

$$
\begin{aligned}
& x^{(0)}(i)=d^{(q)}(i), \\
& M_{\text {symb }}^{\text {layer }}=M_{\text {symb }}^{(q)},
\end{aligned}
$$

and when $v=2[9$, Table 7.3.1.3-1],

$$
\begin{aligned}
& x^{(0)}(i)=d^{(q)}(2 i), \\
& x^{(1)}(i)=d^{(q)}(2 i+1), \\
& M_{\text {symb }}^{\text {layer }}=M_{\mathrm{symb}}^{(q)} / 2 .
\end{aligned}
$$

The modulation symbols are first precoded, then scaled by the amplitude scaling factor for power control, and finally mapped to the REs indexed with $\left(k^{\prime}, l\right)_{p, u}$, in the corresponding Virtual Resource Blocks (VRBs), following the rules [9, Table 8.3.1.5]:

1. Starting from the lowest $k^{\prime}$ and the first PSSCH OFDM symbol that contains Demodulation Reference Signal (DM-RS) for PSSCH, the precoded symbols for the 2ndstage SCI are mapped to the REs that are not assigned to DM-RS, Phase-Tracking Reference Signal (PT-RS), or PSCCH. Mapping of the REs first fills the frequency domain by increasing $k^{\prime}$, and then moves up in time domain by increasing $l$;

2. Starting from the lowest $k^{\prime}$ and $l$, the precoded symbols that are not for the 2nd-stage SCI fills the REs for the PSSCH that are not assigned for the 2nd-stage SCI, DM-RS, PT-RS, or PSCCH, in the increasing order of first $k^{\prime}$ and then $l$. 
Taking the example in Figs. 3 and 4, mapping of the precoded symbols for the 2nd-stage SCI starts in the first available RE at the OFDM symbol $l=3$ in Fig. 3, and $l=1$ in Fig. 4. On the other hand, in both figures, mapping of the precoded symbols for the SL-SCH starts at the first available RE for the PSSCH from $l=1$. Mapping of 2nd-stage SCI symbols, as in Fig. 3, is also illustrated in Fig. 5.

The VRBs above are then mapped to the corresponding PRBs.

\section{PSCCH:}

The PSCCH carries the 1st-stage SCI, which schedules the time and frequency resources for the corresponding PSSCH, as well as the associated 2nd-stage SCI, DM-RS, and configurations such as MCS, PSFCH, etc. The format of the 1st-stage SCI is SCI format 1-A. The UE procedure for PSCCH transmissions is introduced in [10, Section 16.4]. As illustrated in Figs. 3 and 4, PSCCH begins at the second OFDM symbol within a slot, and it may occupy 2 or 3 consecutive symbols in the time domain. In the frequency domain, the number of PRBs allocated for it can be 10,12, 15, 20, or 25 but cannot exceed the number of PRBs assigned to a subchannel. Allocation of time and frequency resources are configured at the RRC [7, Section 6.3.5].

Similar to the processing of the 2nd-stage SCI at the transport layer, the 1st-stage SCI bits are attached with CRC bits, channel coded with Polar coding, and then rate matched [11, Section 8.3]. The rate matched bits are then scrambled and modulated with QPSK at the PHY. The modulation symbols are multiplied with the amplitude scaling factor for power control, and then mapped to the REs for the PSCCH but not for the associated DM-RS symbols. This mapping increases in frequency first and then in time [9, Section 8.3.2].

\section{PSFCH:}

PSFCH is a new PHY channel introduced by NR SL, and is used to carry Sidelink Feedback Control Information (SFCI) (i.e., 1-bit ACK or Negative Acknowledgement (NACK)). Instead of directly sending the 1-bit information bit, a pseudo random sequence is transmitted. A base pseudo random sequence (a low-Peak-to-Average-Power Ratio (PAPR) sequence) is constructed based on the length of the sequence to be generated, the group number, as well as the sequence number within a group [9, Section 5.2.2]. There are a total of 30 groups. The group ID number $u$ and the sequence ID number $v$ within a group can be computed based on $n_{I D}$, which is given by the higher-layer parameter, sl-PSFCH-HopID. If $n_{I D}$ is configured by the higher layer, we have group ID

$$
u=n_{I D} \bmod 30,
$$

and sequence ID $v=0$; otherwise, we have $u=0$ and $v=0$.

To multiplex multiple PSFCH transmissions in the same frequency-time resource, on top of the base sequence, a cyclic shift is applied. The cyclic shift is also generated based on a pseudo random sequence [9, Section 5.2.1] and is determined by the HARQ-ACK information and the resource allocation schemes. To be specific, the phase rotation that results from the cyclic shift is a function of the slot number and the symbol index within 
the slot, along with $m_{0}$ and $m_{c s}$ values determined by the UE [10, Section 16.3], and other parameters, which can be computed as [9, Section 6.3.2.2.2]:

$$
\alpha_{l}=\frac{2 \pi}{N_{S C}^{R B}}\left(\left(m_{0}+m_{c s}+m_{i n t}+n_{c s}\left(n_{s, f^{\prime}}^{\mu} l+l^{\prime}\right)\right) \quad \bmod N_{S C}^{R B}\right),
$$

where

$$
n_{c s}\left(n_{s, f^{\prime}}^{\mu}, l\right)=\sum_{m=0}^{7} 2^{m} c\left(8 N_{s y m b}^{s l o t} n_{s, f}^{\mu}+8 l+m\right) .
$$

Here, $m_{\text {int }}=0, l$ is the OFDM symbol number in the PSFCH transmission, $l^{\prime}$ is the index of the OFDM symbol in the slot that corresponds to the first OFDM symbol of the PSFCH in the slot, $n_{s, f}^{u}$ is the slot number in the radio frame, $m_{c s}$ is the information bit to be transmitted, and $m_{0}$ depends on the cyclic shift pair index in the cyclic shift pairs configured for the resource pool. Also, $c(\cdot)$ is the pseudo random sequence defined by a length-31 Gold sequence and initialized by $c_{\text {init }}$. If $n_{I D}$ is configured by a higher layer, $c_{\text {init }}=n_{I D}$; otherwise $c_{\text {init }}=0$. After the sequence is generated, it shall be power scaled to conform to the transmit power specification and mapped to one OFDM symbol. Note that the resource elements used for the PSFCH in the first OFDM symbol, including the reference signals, shall be duplicated in the immediately preceding OFDM symbol.

\subsubsection{Physical Reference Signals}

\section{DM-RS for PSSCH:}

In an OFDM symbol for the PSSCH and its associated DM-RS, the frequency locations for DM-RS has an index of $k=0,2,4,8, \ldots$, where $k$ is the index for an RE relative to subcarrier 0 in CRB 0 , and $k$ should fall within the CRBs allocated for the PSSCH transmission. As discussed above, in the first symbol that contains the PSSCH and its associated DM-RS (in ascending order), the subcarriers for PSSCH are first filled with modulation symbols for the 2nd-stage SCI, and then filled by modulation symbols for the SL-SCH. Fig. 5 illustrates their inter-dependencies in the frequency domain.

Time locations of the OFDM symbols that contain the PSSCH DM-RS are determined by 1) the total number of OFDM symbols for the PSSCH, its associated PSCCH and AGC, 2) the number of PSCCH symbols, and 3) the number of PSSCH DM-RS symbols, using the reference from [9, Table 8.4.1.1.2-1]. Figs. 3 and 4 provide two examples of time location allocations, with a different number of PSCCH, PSSCH and DM-RS symbols. Another example is detailed in Section 5.3.

Prior to being mapped to physical resources, the PSSCH DM-RS sequence is generated at the PHY and scaled by amplitude scaling factor for power control.

\section{DM-RS for PSCCH:}

Similar to the DM-RS for thePSSCH, the sequence for PSCCH DM-RS is also generated at the PHY and scaled for power control before being mapped to physical resources. 


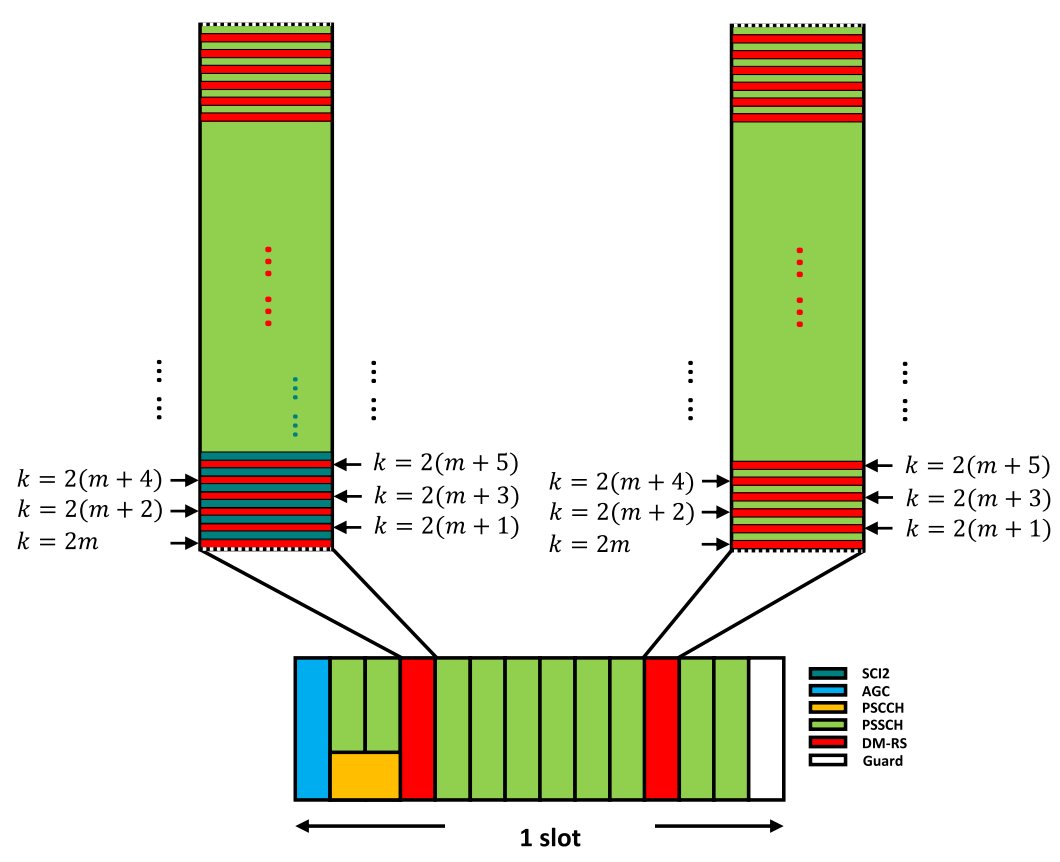

Fig. 5. Allocation of PSSCH DM-RS, 2nd-stage SCI and SL-SCH modulation symbols.

The frequency locations of PSCCH DM-RS REs are defined as:

$$
\begin{aligned}
k & =n N_{\mathrm{sc}}^{\mathrm{RB}}+4 k^{\prime}+1, \\
k^{\prime} & =0,1,2, \\
n & =0,1, \ldots,
\end{aligned}
$$

where $k$ is the subcarrier index in reference to subcarrier 0 in CRB 0 , and falls within the CRBs allocated for the PSCCH transmission. Therefore, we can derive $k$ from Eq. 14 as $k=4 m+1,4(m+1)+1,4(m+2)+1, \ldots$, as illustrated in Fig. 6, where $4 m+1$ corresponds to the first RE allocated for the PSCCH DM-RS.

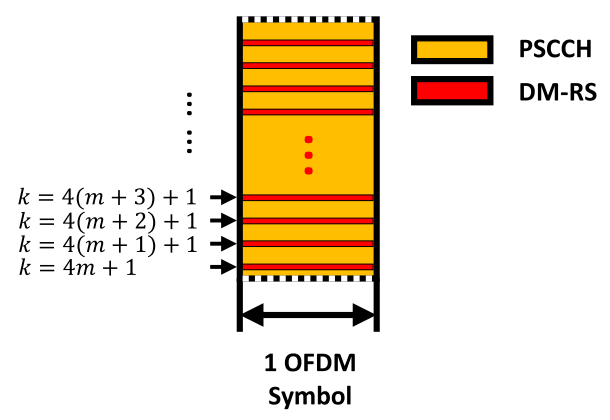

Fig. 6. Frequency locations of PSCCH DM-RS.

PT-RS for PSSCH: 
The PT-RS is a newly introduced Reference Signal in 5G NR. The purpose of PT$\mathrm{RS}$ is to track phase changes and compensate phase noise during transmissions, which is more prominent for higher carrier frequencies. Therefore, the PT-RS is only supported for Frequency Range 2 (FR2). If the PT-RS is configured to be present, it should be transmitted in the resource blocks used for the PSSCH, and the time density and frequency of the PT$\mathrm{RS}$ can be configured by the upper layer and is configured per resource pool [12, Section 8.2.3]. The sequence generation and the details of how the reference signal is mapped to physical resources can be found in [9, Section 8.4.1].

\section{Channel-State Information Reference Signal (CSI-RS):}

The CSI-RS is used for channel sounding. The receiving device measures the received CSI-RS, then reports back the Channel State Information (CSI) to the transmitter via the PSSCH. The CSI-RS is configurable in both the time and frequency domain, and can be used to provide fine channel state information. Therefore, the CSI-RS can support mobility and beamforming. For SL, the CSI-RS is configured with a maximum of two antenna ports, and is transmitted in every resource block within the bandwidth CSI-RS being configured [9, Section 8.4.1.5].

\section{S-SS/PSBCH Block:}

A slot that transmits the S-SS/PSBCH block is also known as a Sidelink Synchronization Signal Block (S-SSB). It consists of PSBCH, SPSS and SSSS symbols. The period of the S-SSB transmission is 16 frames [10, Section 16.1], and within each period, the number of S-SSB blocks $N_{\text {period }}^{\text {S-SSB }}$ is configured at the RRC [7, Section 6.3.5]. The range of choices for $N_{\text {period }}^{\mathrm{S}-\mathrm{SSB}}$ varies with $\mu$ and the frequency range. Their relationships are listed in Table 2. The time offset, $N_{\text {offset }}^{\text {S-SSB }}$, and time interval, $N_{\text {interval }}^{\text {S-SSB }}$, within a period are also configured at the RRC. Both are measured in terms of a slot. The relationship between slot index $i_{\text {slot }}^{\mathrm{S}-\mathrm{SSB}}$, S-SS/PSBCH block index $i_{\text {S-SSB }}, N_{\text {offset }}^{\text {S-SSB }}$ and $N_{\text {interval }}^{\text {S-SSB }}$ are illustrated in Fig. 7 (with $\mu=1$ ) and is expressed as [10, Section 16.1], [7, Section 6.3.5]:

$$
i_{\text {slot }}^{\mathrm{S}-\mathrm{SSB}}=N_{\mathrm{offset}}^{\mathrm{S}-\mathrm{SSB}}+N_{\text {interval }}^{\mathrm{S}-\mathrm{SSB}} \cdot i_{\mathrm{S}-\mathrm{SSB}},
$$

where index 0 of $i_{\text {slot }}^{\mathrm{S}-\mathrm{SSB}}$ coincides with a first slot of a frame with subframe number

$$
i_{\mathrm{SFN}} \bmod 16=0
$$

and

$$
\begin{aligned}
& 0 \leq N_{\text {offset }}^{\mathrm{S}-\mathrm{SSB}} \leq 1279 \\
& 0 \leq N_{\text {interval }}^{\mathrm{S}-\mathrm{SSB}} \leq 639 \\
& 0 \leq i_{\mathrm{S}-\mathrm{SSB}} \leq N_{\text {period }}^{\mathrm{S-SSB}}-1 .
\end{aligned}
$$

The SPSS and SSSS are generated using the M-sequence and Gold sequence, respectively. PHY SL identities are used while generating the sequences. 


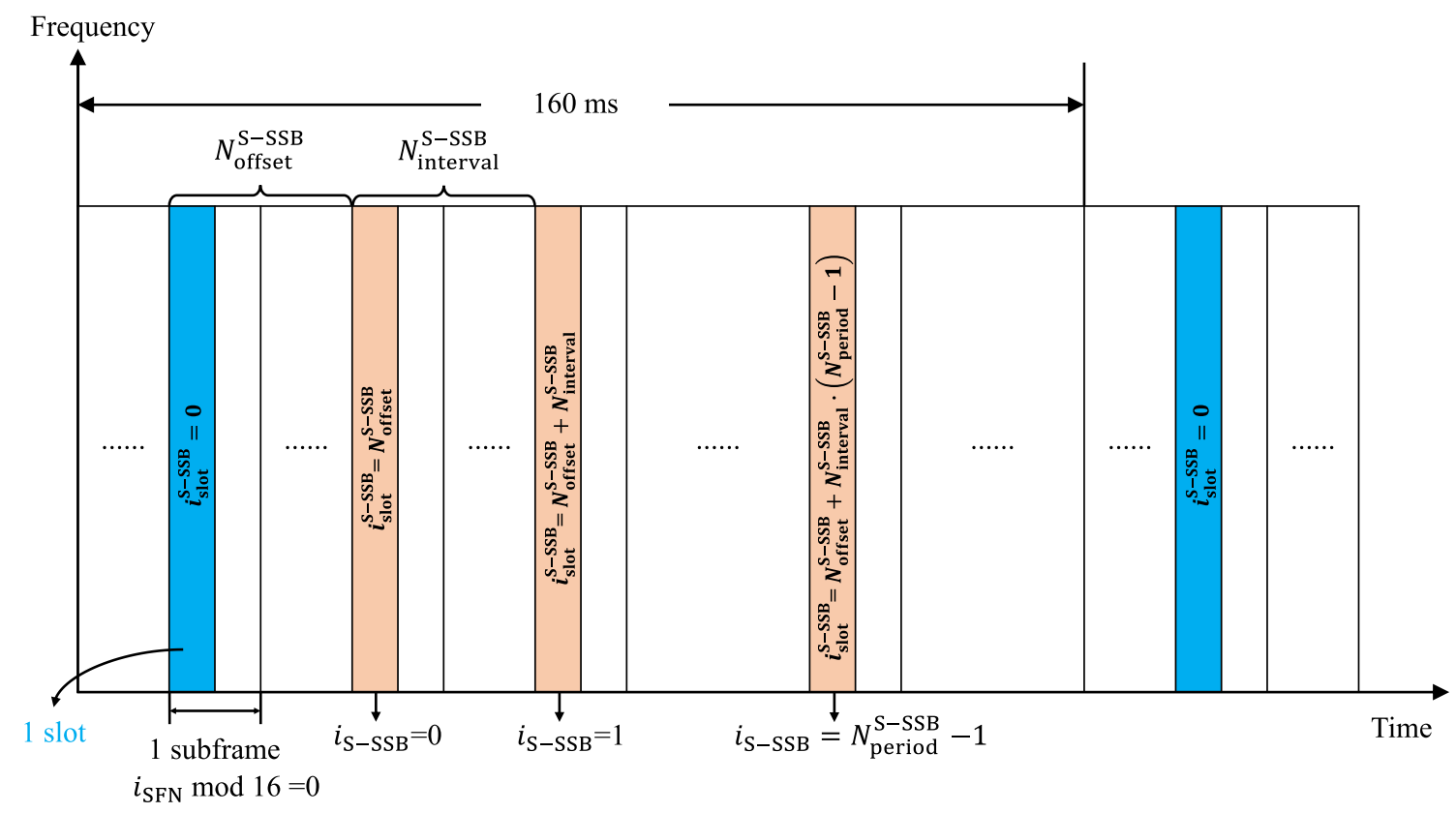

Fig. 7. Transmission of S-SS/PSBCH blocks with $\mu=1$.

Table 2. Choices of $N_{\text {period }}^{\mathrm{S}-\mathrm{SSB}}$ vs. $\mu$ and frequency range. [7, Section 6.3.5]

\begin{tabular}{|c|c|c|l|}
\hline $\begin{array}{c}\text { Frequency } \\
\text { Range }\end{array}$ & $\mu$ & SCS $[\mathrm{kHz}]$ & \multicolumn{1}{|c|}{$N_{\text {period }}^{\text {S-SSB }}$} \\
\hline \multirow{3}{*}{ FR1 } & 0 & 15 & $\{1\}$ \\
\cline { 2 - 4 } & 1 & 30 & $\{1,2\}$ \\
\cline { 2 - 4 } & 2 & 60 & $\{1,2,4\}$ \\
\hline \multirow{2}{*}{ FR2 } & 2 & 60 & $\{1,2,4,8,16,32\}$ \\
\cline { 2 - 4 } & 3 & 120 & $\{1,2,4,8,16,32,64\}$ \\
\hline
\end{tabular}

Similar to 1st- and 2nd-stage SCIs, at the transport layer, the Sidelink Broadcast Channel (SL-BCH) payload is generated, attached with CRC bits, channel coded with Polar coding, and then rate matched [11, Section 8.1]. At the PHY, the block of bits are scrambled and modulated with QPSK before being mapped to the physical resources in an S-SSB slot [9, Sections 8.3.3, 8.4.3].

The DM-RS for the PSBCH is assigned to all PSBCH symbols. The PSBCH DM-RS, along with the SPSS and SSSS, are first scaled for power control, and then assigned to the resources following [9, Table 8.4.3.1-1]. A slot format of an S-SSB is illustrated in Fig. 8, where a normal cyclic prefix is assumed:

1. For a normal cyclic prefix, as shown in Fig. 8, the number of S-SS/PSBCH OFDM symbols is 13 , whereas it is 11 for an extended cyclic prefix, and the last OFDM symbol in the slot is left unused as a guard symbol for both cases [9, Section 8.4.3.1]. 


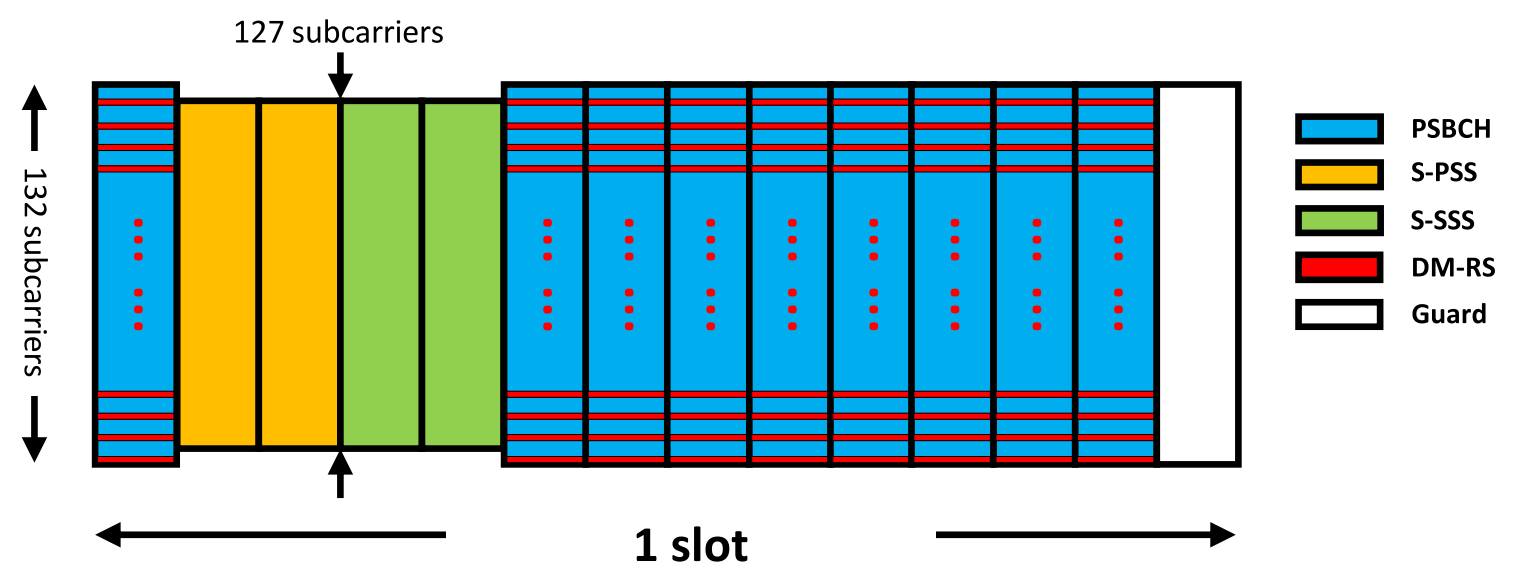

Fig. 8. A slot format of S-SSB with normal cyclic prefix.

2. In the frequency domain, 132 subcarriers are assigned for the OFDM symbols, with $k=0,1, \ldots, 131$. Each PSBCH symbol, as well as its associated DM-RS, occupies all the 132 subcarriers. For the SPSS and SSSS symbols, the subcarriers $k=$ $1,2, \ldots, 127,128$ are occupied, and the rest are set to zero [9, Table 8.4.3.1-1].

3. In the time domain, SPSSs are assigned to the 2nd and 3rd OFDM symbols, with indexes $l=1$ and $l=2$, respectively, whereas the SSSS follows the SPSS and are assigned to the OFDM symbols with indexes $l=3$ and $l=4$. PSBCH symbols are assigned to the rest of the assigned S-SS/PSBCH time resources, and the guard symbol follows the last PSBCH symbol.

4. In each PSBCH symbol, the associated DM-RS REs are assigned to the subcarriers with indexes $4 n$, where $n=0,1, \ldots, 32$ [9, Table 8.4.3.1-1].

In order to determine the location of the S-SSB in the frequency domain, the RX UE is configured with an absolute frequency via the parameter sl-AbsoluteFrequencySSB in the RRC configuration [10, Section 16.1], which provides the position of the subcarrier $k=66$ in the S-SSB.

\subsection{MIMO and Beamforming}

Multiple-Input and Multiple-Output (MIMO) technology can facilitate beamforming, spatial multiplexing, or spatial diversity by using multiple transmit or receiver antennas or an antenna array with a large number of antenna elements, and it supports different transceiver architectures such as analog, digital, or hybrid. In NR, MIMO standardization focuses on the following areas: MIMO transmission schemes, CSI measurement and report, reference signal design, and beam management [13].

Two schemes are defined for DL transmission: (i) closed-loop transmission (scheme 1), where the DM-RS is transmitted using the same precoding matrix as data transmission; (ii) 
open-loop and semi-open loop transmission (scheme 2), where data and DM-RS may use different precoding matrices. Note that since NR minimizes the always-on Cell Reference Signal (CRS) usage, DM-RS will be used for channel estimation and data demodulation for DL signals. For open-loop transmission, a transmitter can use a fixed precoding matrix without the CSI knowledge. Therefore, in a closed-loop scenario, the receiver needs to send back a Precoder Matrix Indicator (PMI), while, in an open-loop transmission, the receiver may only report Channel Quality Indication (CQI) and Rank Indication (RI). In the DL transmission, as of Rel-16, to maintain the DM-RS signal's orthogonality, a maximum of 8 layers can be supported for Single-User MIMO (SU-MIMO), and a maximum of 12 layers can be supported for Multi-User MIMO (MU-MIMO).

For UL transmissions, a maximum of 4 layers can be supported by NR UL. Compared to LTE UL, which only supports Single-Carrier FDMA (SC-FDMA) waveform, NR UL supports both SC-FDMA and OFDM with CP waveforms. It is worth noting that the closedloop MIMO transmission only supports the OFDM waveform. In addition to the proposed codebook-based transmission in LTE, the non-codebook approach can also be used in UL transmissions. For example, in a TDD system, a UE can design its precoding matrix based on channel reciprocity.

For SL, as of Rel-16, a maximum of two layers is supported in the NR SL and its MIMO scheme is limited to $2 \times 2$. More layers are expected to be supported in future releases [2].

In terms of the CSI reporting and feedback, NR MIMO can support two types of CSI feedback in the Frequency Division Multiplexing (FDD) system. The type I CSI feedback, which is also supported in LTE, allows the receiver to feedback a PMI matrix to the transmitter by identifying a preferred precoding matrix in a standardized codebook. Beyond the type I codebook, NR also supports the type II CSI feedback, which has a higher resolution than the type I codebook. Type II feedback has the potential to improve the average user throughput significantly (at least $30 \%$ ) over LTE Rel.14, but at the expense of high feedback overhead [14]. The goal of type II feedback is to improve MU-MIMO performance with an increased number of transceivers.

NR expands its supported carrier frequency to cover a much higher frequency spectrum, such as Millimeter Wave (mmWave). In order to compensate for the large propagation loss occurring during the high-frequency transmission, antennas with a large number of elements are often used to beamform the signal and provide additional directional gain. On the other hand, thanks to the high frequency, antenna size can be significantly reduced, and it is feasible to pack more antennas in a small form factor.

Based on the limited study conducted by 3GPP RAN1, beamforming is beneficial to SL communication, especially in FR2 [15]. When a UE is equipped with one or multiple antenna arrays in direct communication mode, a simple phase shift or a complex gain value can be applied to each antenna element to form a beam or multiple beams. A larger antenna size will lead to higher directional gain, but the coverage angle will be reduced. Therefore, to provide a good coverage for the area, multiple beams may be required. In order to maintain the communication, the beam needs to be monitored, and beam failure and recovery need to be addressed in a timely manner. The beam management procedure, a set of L1 
and L2 procedures to acquire and maintain beams between Transmitter and Receiver Points (TRPs) and UEs for DL and UL communications is defined in [16]. The beam management procedure covers beam determination, beam measurement, beam reporting, as well as beam sweeping. Beamforming and MIMO study continues to be carried out in Rel-17, which includes enhancements to multi-beam operation, distributed multiple antennas at the transmitter and receiver, the Sounding Reference Signal (SRS), and the CSI measurement and reporting [17].

\subsection{Summary}

In summary, NR SL introduces numerous new PHY layer features, including configurable numerology to allow resource usage to be more flexible in both time and frequency domain. NR also supports new coding schemes, i.e., LDPC for data channels that reduces decoding complexity and polar code for control channels that efficiently handles small-size block transmission. In terms of physical channels and physical reference signaling, NR adds a new physical layer feedback channel, i.e., PSFCH, which can reduce blind transmissions and improve spectrum efficiency. With respect to power efficiency, NR introduces BWP such that devices do not have to monitor the whole carrier bandwidth for the control signals. MIMO and beamforming are important areas for NR, but more studies are needed to determine under which conditions they could be used for SL communication..

\section{Capacity}

\subsection{Introduction}

One major performance metric for SL communications is capacity, which in this context refers to the maximum achievable data rate. Compared with LTE SL, NR SL supports multiple features that are expected to improve capacity significantly. Some major ones are up to two-layer spatial multiplexing, higher modulation and coding schemes, and configurable HARQ transmissions. In addition, NR also supports a flexible OFDM numerology to meet different types of service requirements.

In this section we provide our initial investigation on NR SL capacity and its comparison with LTE. We first look at LTE SL capacity, then study NR SL capacity and its improvement brought by different features. The public safety band, Band 14 / Band n14 with $10 \mathrm{MHz}$ bandwidth, is selected for numerical analysis. While NR SL supports unicast, multicast, and broadcast, in this analysis we focus on unicast and will extend the work to multicast and broadcast in the future when the related standards are relatively mature.

\subsection{LTE Capacity}

Communication over LTE SL is periodic in the time domain. An SL period can be configured to last from $40 \mathrm{~ms}$ to $320 \mathrm{~ms}$, with a set of predefined values by the RRC layer [18, Section 6.3.8]. The PSCCH is used to indicate how, where, and when transmissions will 
occur in the PSSCH. The PSCCH and PSSCH are multiplexed in the time domain, with the PSCCH always preceding the PSSCH, as depicted in Fig. 9.

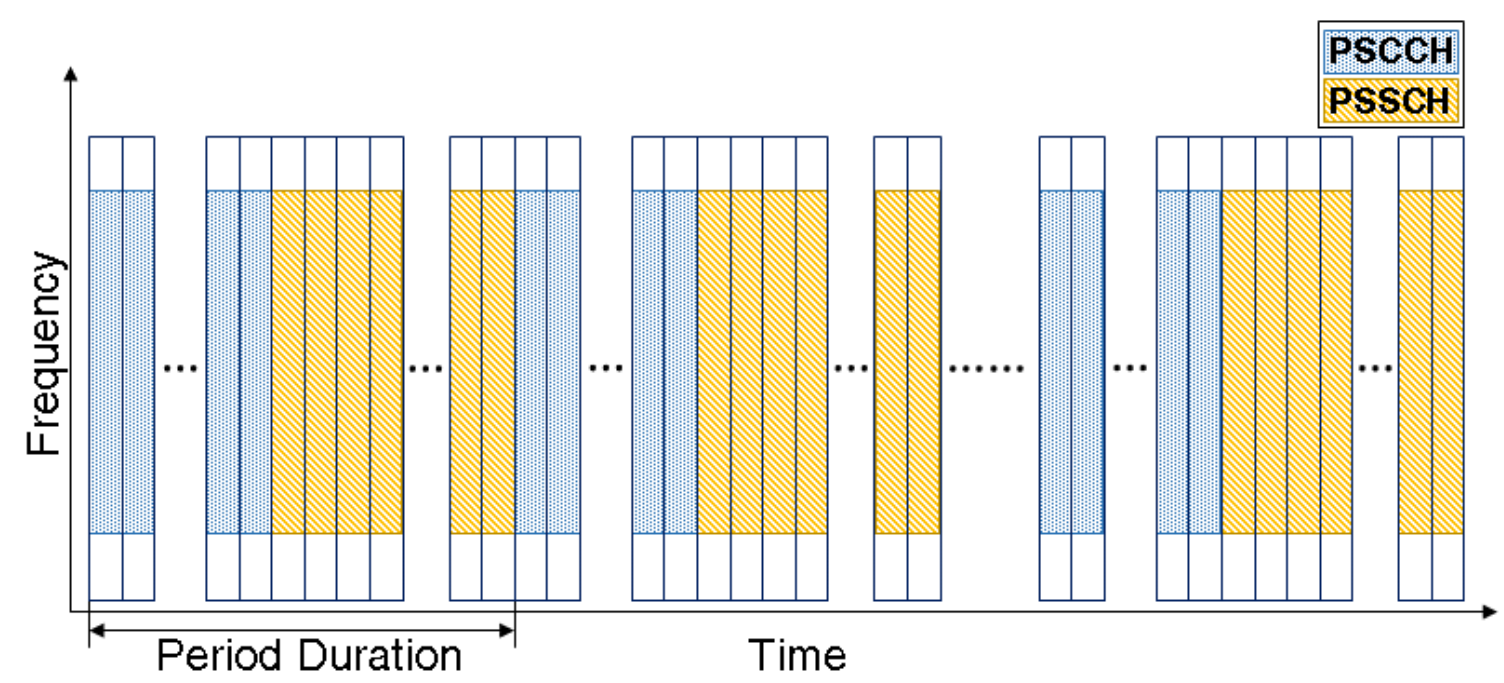

Fig. 9. LTE's SL periodic transmission of PSCCH and PSSCH [19].

Both, the PSCCH and PSSCH, have a set of resources configurable in the time and frequency domain. Within the SL period, the PSCCH can be configured to last from $2 \mathrm{~ms}$ and up to $40 \mathrm{~ms}$, leaving the remaining time available for the PSSCH.

To compute the maximum SL capacity, without considering overhead from retransmission protocols (e.g., HARQ), the shortest PSCCH (2 ms) is selected. This maximizes the time available for the PSSCH. For example, in a $40 \mathrm{~ms}$ SL period, $2 \mathrm{~ms}$ would be reserved for PSCCH and the remaining $38 \mathrm{~ms}$ would be for the PSSCH. Assuming a $10 \mathrm{MHz}$ bandwidth is available, $50 \mathrm{PRBs}$, and no time restriction (i.e., all subframes ${ }^{1}$ are made available for SL transmissions) within the PSSCH, we compute the SL capacities for all the possible SL period lengths. The highest MCS supported by SL, MCS 20, is used in the analysis. The capacities at the physical layer are presented in Fig. 10. A clear trend is observed, with longer SL period lengths there is less overhead incurred by fewer transmissions of PSCCHs, hence the higher capacities. It is worth noting that, even when the difference in $\mathrm{Mbit} / \mathrm{s}$ is visually notorious given the scale used, the relative difference is not that significant across all SL periods analyzed. A delta of $0.94 \mathrm{Mbit} / \mathrm{s}$ is observed from the shortest to the longest SL period, which provides a capacity increase that is less than $5 \%$.

LTE SL uses HARQ with no feedback. Four blind HARQ processes are employed for every TB transmission. The transmission of a TB would only occur if there are enough time resources for all the HARQ processes associated with the TB to be transmitted, i.e., four transmission opportunities per TB. After considering the overhead created by HARQ blind retransmissions in SL, we can compute the actual capacity for SL, presented in Fig. 11. A delta of $0.47 \mathrm{Mbit} / \mathrm{s}$ is observed from the shortest to the longest SL period, which is

${ }^{1}$ In LTE, a subframe is $1 \mathrm{~ms}$ long. 


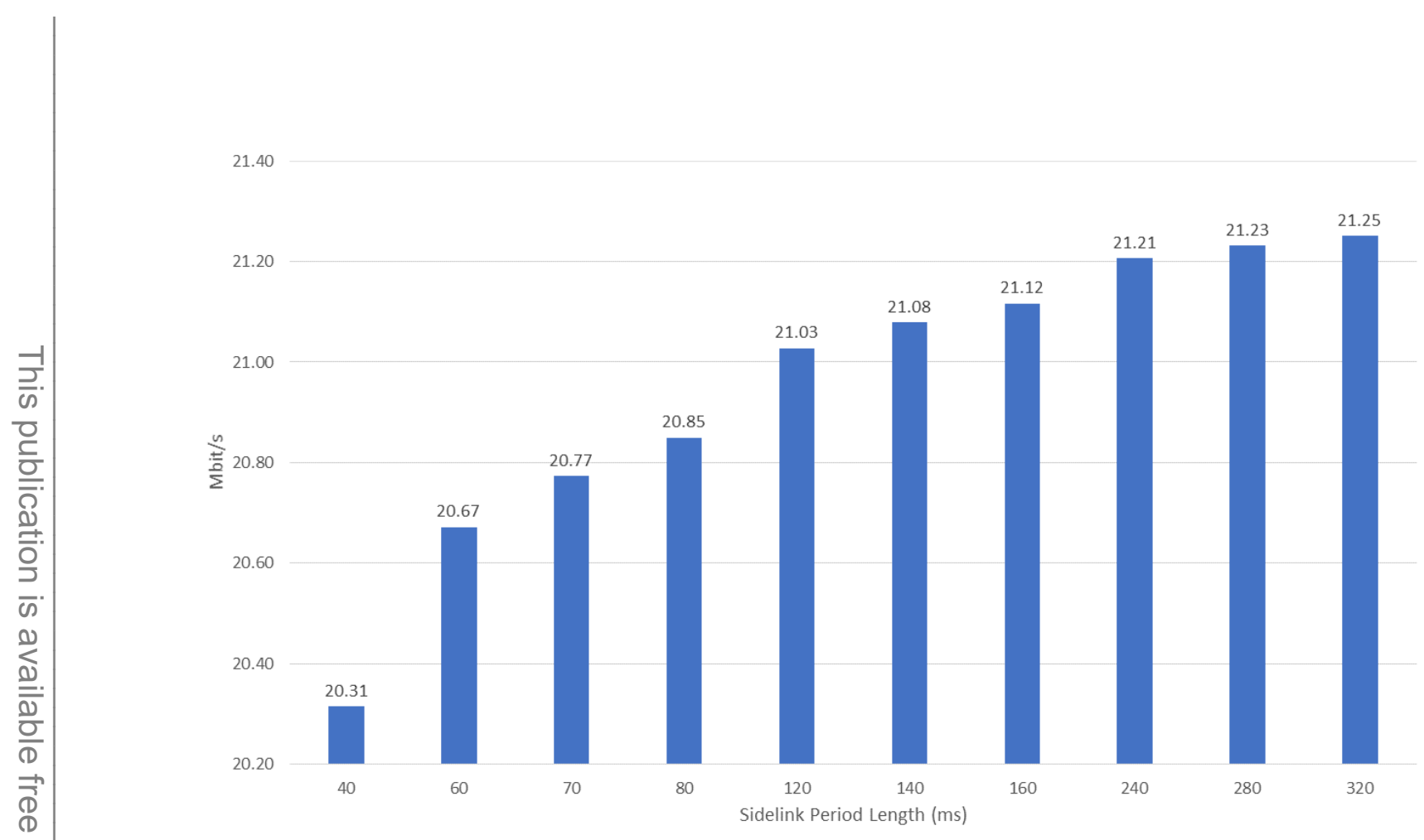

Fig. 10. LTE SL capacity, without considering overhead from HARQ, achieved for each period length, with a 2 ms long PSCCH.

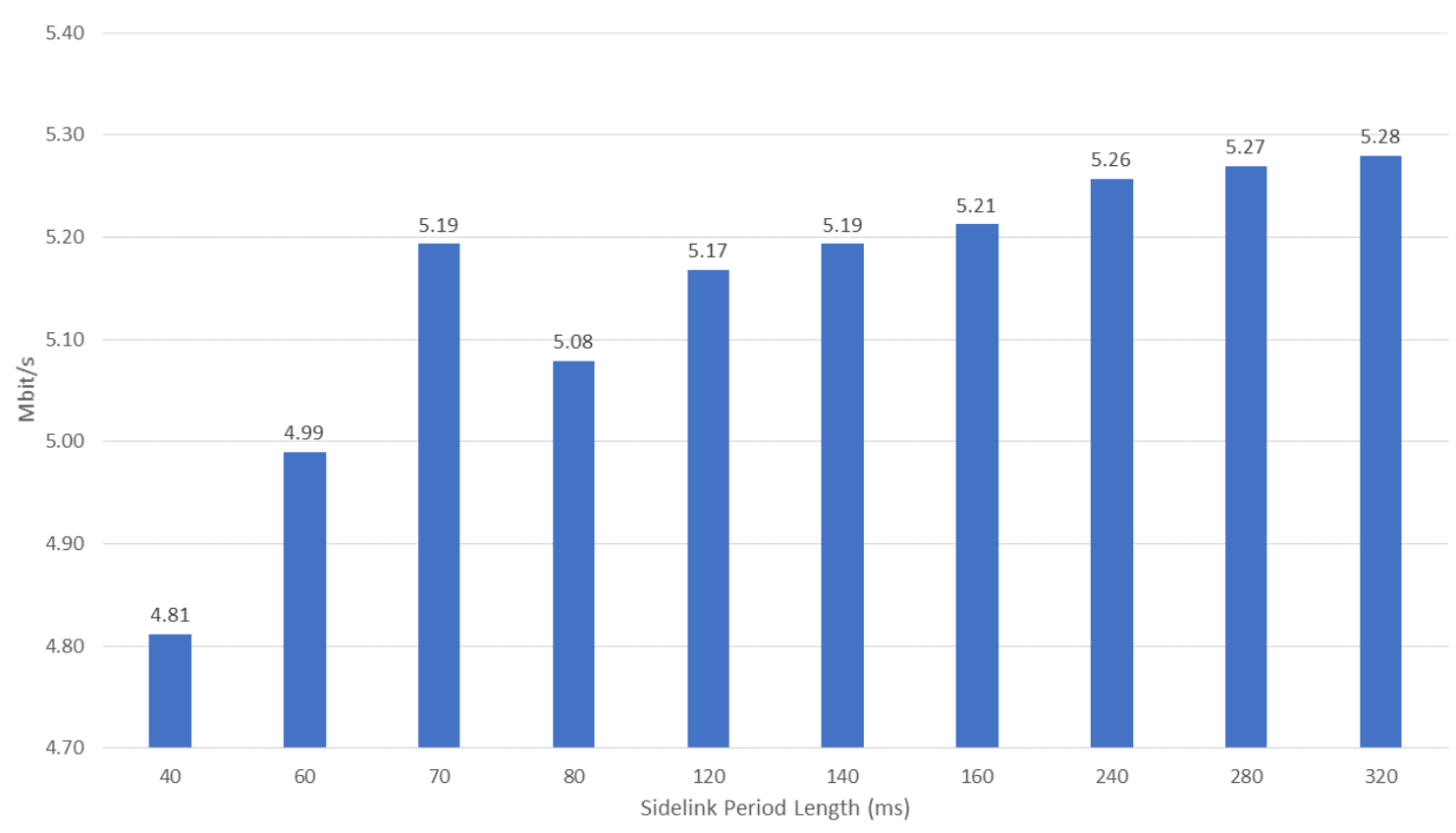

Fig. 11. LTE SL capacity, considering overhead from HARQ, achieved for each period length, considering HARQ blind retransmissions. The PSCCH is $2 \mathrm{~ms}$ long and the PSSCH lasting the remaining period length, respectively. 
just under a $10 \%$ capacity increase. The capacities are also affected by the length of the PSSCH not being divisible by a factor of four (the number of blind HARQ transmissions), which leaves unused transmission opportunities. With our configuration assumptions, only the $70 \mathrm{~ms}$ period with a $68 \mathrm{~ms}$ long PSSCH leaves no unused transmission opportunities, making this configuration stand out.

\subsection{NR Capacity}

Different from LTE SL, NR SL introduces a number of features that improve the capacity significantly, such as spatial multiplexing, higher modulation and coding schemes, and a configurable HARQ. NR SL also supports different numerologies, similar to the NR Uu interface. With these factors considered and together with the total available resources, NR SL capacity can be calculated as shown below:

$$
\text { data rate }(\text { in Mbit } / s)=10^{-6} \cdot v_{\text {layers }} \cdot Q_{m} \cdot f \cdot R_{\text {max }} \cdot \frac{N_{P R B}^{B W, \mu} \times 12}{T_{S}^{\mu}} \cdot(1-O H),
$$

where $v_{\text {layers }}$ is the spatial multiplexing number of layers, $Q_{m}$ is the modulation order, $f$ is the scaling factor, $R_{\max }$ is the coding rate, $N_{P R B}^{B W, \mu}$ is the number of PRBs for a specific bandwidth $B W$ and numerology $\mu, 12$ is the number of sub-carriers per physical resource block as specified by $3 \mathrm{GPP}, T_{s}^{\mu}$ is the symbol duration time in seconds for numerology $\mu$, $\mathrm{OH}$ is the overhead ratio, and the number of transmissions is one.

While Eq. (18) seems the same as the data rate formula in 3GPP Technical Specification (TS) 38.306 [20, Section 4.1], note that here $O H$ is not necessarily independent of the bandwidth or numerology, which will manifest in the later overhead analysis. Also, while Eq. (18) is used as the base of the later analysis and addresses the configuration with the number of transmissions set to one, it will be extended to include the configuration with blind retransmissions. In the following we will examine each parameter first, then analyze overheads and HARQ configurations, followed by the resulting capacity numbers.

\subsubsection{Parameter Settings}

To evaluate capacity, the value for each parameter in Eq. (18) is selected to maximize the resulting data rate, as described below.

$v_{\text {layers }}$ : for NR SL unicast transmissions, the maximum number of layers supported for spatial multiplexing is two [9, Section 8.3.1.3]. Hence we choose $v_{\text {layers }}=2$.

$Q_{m}$ and $R_{\text {max }}$ : NR SL reuses two of the NR downlink MCS tables for its transmissions [12, Section 8.3.1.3], namely 64 QAM table and 256 QAM table, respectively. The 64 QAM table is the default MCS table, while the 256 QAM table may be selected via sl-MCS-Table from the higher layer based on factors, such as channel conditions and UE capabilities. Table 3 and Table 4 show part of the two tables in [12]. It can be seen that the highest MCS has a modulation order of six or eight per MCS table selected, with coding rate $948 / 1024$. This gives $Q_{m}=6$ or 8 and $R_{\max }=948 / 1024$. 
Table 3. 64 QAM MCS Table [9, Table 5.1.3.1-1].

\begin{tabular}{|c|c|c|c|}
\hline $\begin{array}{c}\text { MCS Index } \\
I_{M C S}\end{array}$ & $\begin{array}{c}\text { Modulation Order } \\
Q_{m}\end{array}$ & $\begin{array}{c}\text { Target code Rate } \\
\mathbf{R} \mathbf{x}[\mathbf{1 0 2 4}]\end{array}$ & $\begin{array}{c}\text { Spectral } \\
\text { efficiency }\end{array}$ \\
\hline 0 & 2 & 120 & 0.2344 \\
\hline 1 & 2 & 157 & 0.3066 \\
\hline$\ldots$ & $\ldots$ & $\ldots$ & $\ldots$ \\
\hline 27 & 6 & 910 & 5.3320 \\
\hline 28 & 6 & 948 & 5.5547 \\
\hline 29 & 2 & \multicolumn{2}{|c|}{ reserved } \\
\hline$\ldots$ & $\ldots$ & \multicolumn{2}{|c}{} \\
\hline
\end{tabular}

Table 4. 256 QAM MCS Table [9, Table 5.1.3.1-2].

\begin{tabular}{|c|c|c|c|}
\hline $\begin{array}{c}\text { MCS Index } \\
I_{M C S}\end{array}$ & $\begin{array}{c}\text { Modulation Order } \\
Q_{m}\end{array}$ & $\begin{array}{c}\text { Target code Rate } \\
\mathbf{R ~ x ~ [ 1 0 2 4 ]}\end{array}$ & $\begin{array}{c}\text { Spectral } \\
\text { efficiency }\end{array}$ \\
\hline 0 & 2 & 120 & 0.2344 \\
\hline 1 & 2 & 193 & 0.3770 \\
\hline$\ldots$ & $\ldots$ & $\ldots$ & $\ldots$ \\
\hline 26 & 8 & 916.5 & 7.1602 \\
\hline 27 & 8 & 948 & 7.4063 \\
\hline 28 & 2 & \multicolumn{2}{|c|}{ reserved } \\
\hline$\ldots$ & $\ldots$ & \multicolumn{2}{|c|}{} \\
\hline
\end{tabular}

$f$ : the scaling factor was introduced mainly to capture capability mismatch between the baseband capabilities and Radio Frequency (RF) capabilities, and/or hardware resource sharing between LTE and NR [21]. Here we consider Band n14 NR. There is no other component carrier and no sharing between NR and LTE. Hence the scaling factor $f=1$.

$N_{P R B}^{B W, \mu}$ and $T_{s}^{\mu}$ : Table 5.3.5-1 in [6], partly shown in Table 5, provides channel bandwidths for each NR band and corresponding SCS. Per this table, for the considered Band n14 $10 \mathrm{MHz}$, two numerology configurations are supported, 0 and 1, as listed in Table 6. This leads to normal CP [9, Table 8.2.2-1], and the maximum number of 52 PRBs and 24 PRBs, respectively [6, Table 5.3.2-1]. The corresponding symbol duration, in seconds, can be calculated from the formula:

$$
T_{s}^{\mu}=10^{-3} /\left(14 \times 2^{\mu}\right) ;
$$

where $\mu$ is the numerology, $10^{-3}$ is the time in seconds for one subframe, $2^{\mu}$ is the number of slots per subframe, and 14 is the number of symbols per slot.

The resulting values per numerology are also listed in Table 6. Note that a different numerology leads to a different number of PRBs and symbol duration, as well as their ratio $\frac{N_{P R B}^{B W, \mu}}{T_{S}^{\mu}}$, which directly affects the resulting capacity (Eq. (18)).

It is worth mentioning that, as discussed in Section 3.3, resources are allocated to users 
Table 5. NR Channel Bandwidth Table [9, Table 5.3.5-1]

\begin{tabular}{|c|c|c|c|c|c|c|c|c|c|c|c|c|c|c|}
\hline \multicolumn{15}{|c|}{ NR band / SCS / UE Channel bandwidth } \\
\hline $\begin{array}{c}\text { NR } \\
\text { Band }\end{array}$ & $\begin{array}{l}\text { SCS } \\
\text { kHz }\end{array}$ & $\begin{array}{c}5 \\
\mathrm{MHz}\end{array}$ & $\begin{array}{c}10 \\
\mathrm{MHz}\end{array}$ & $\begin{array}{c}15 \\
\text { MHz }\end{array}$ & $\begin{array}{c}20 \\
\mathrm{MHz}\end{array}$ & $\begin{array}{c}25 \\
\text { MHz }\end{array}$ & $\begin{array}{c}\text { 30 } \\
\text { MHz }\end{array}$ & $\begin{array}{c}40 \\
\text { MHz }\end{array}$ & $\begin{array}{c}\mathbf{5 0} \\
\text { MHz }\end{array}$ & $\begin{array}{c}60 \\
\text { MHz }\end{array}$ & $\begin{array}{c}\mathbf{7 0} \\
\mathrm{MHz}\end{array}$ & $\begin{array}{c}\mathbf{8 0} \\
\mathrm{MHz}\end{array}$ & $\begin{array}{c}90 \\
\mathrm{MHz}\end{array}$ & $\begin{array}{c}100 \\
\mathrm{MHz}\end{array}$ \\
\hline \multicolumn{15}{|c|}{$\ldots$} \\
\hline \multirow{3}{*}{ n14 } & 15 & Yes & Yes & & & & & & & & & & & \\
\hline & 30 & & Yes & & & & & & & & & & & \\
\hline & 60 & & & & & & & & & & & & & \\
\hline \multicolumn{15}{|c|}{$\ldots$} \\
\hline \multirow{3}{*}{ n38 } & 15 & Yes & Yes $^{10}$ & Yes & Yes $^{10}$ & Yes & Yes $^{10}$ & Yes $^{10}$ & & & & & & \\
\hline & 30 & & Yes $^{10}$ & Yes & Yes $^{10}$ & Yes & Yes $^{10}$ & Yes $^{10}$ & & & & & & \\
\hline & 60 & & Yes $^{10}$ & Yes & Yes $^{10}$ & Yes & Yes $^{10}$ & Yes $^{10}$ & & & & & & \\
\hline \multicolumn{15}{|c|}{$\ldots$} \\
\hline \multirow{3}{*}{$\mathrm{n} 47$} & 15 & & Yes $^{10}$ & & Yes $^{10}$ & & Yes $^{10}$ & Yes $^{10}$ & & & & & & \\
\hline & 30 & & Yes $^{10}$ & & Yes $^{10}$ & & Yes $^{10}$ & Yes $^{10}$ & & & & & & \\
\hline & 60 & & Yes $^{10}$ & & Yes $^{10}$ & & Yes $^{10}$ & Yes $^{10}$ & & & & & & \\
\hline \multicolumn{15}{|c|}{$\cdots$} \\
\hline
\end{tabular}

per subchannel, and there is a discrete set for subchannel sizes. This could lead to leftover RBs inside the resource pool that cannot be used. In our study, however, we use the maximum number of PRBs within the bandwidth to simplify the context.

Table 6. Configurations for Band n14 10 MHz NR SL

\begin{tabular}{|r|r|r|r|c|}
\hline Numerology & $\begin{array}{r}\text { Sub-carrier } \\
\text { Spacing }\end{array}$ & $\begin{array}{r}\text { Number } \\
\text { of PRBs }\end{array}$ & $\begin{array}{r}\text { Symbol Duration } \\
\text { in microseconds }\end{array}$ & $\frac{N_{P R B}^{B W, \mu}}{T_{s}^{\mu}}$ \\
\hline$\mu=0$ & $15 \mathrm{kHz}$ & 52 & 71.43 & 0.73 \\
\hline$\mu=1$ & $30 \mathrm{kHz}$ & 24 & 35.71 & 0.67 \\
\hline
\end{tabular}

As a recap, Table 7 summarizes the values of each parameter discussed above. The numbers will be used in the NR capacity computation later.

Table 7. Parameter Settings for NR Capacity

\begin{tabular}{|l|r|r|r|r|r|r|l|}
\hline Parameter & $v_{\text {layers }}$ & $Q_{m}(\mathrm{MCS}$ Table $)$ & $f$ & $R_{\max }$ & numerology & $\begin{array}{l}\text { Number } \\
\text { of PRBs } \\
N_{P R B}^{B W, \mu}\end{array}$ & $\begin{array}{l}\text { Symbol Duration } \\
T_{s}^{\mu} \\
\text { in microseconds }\end{array}$ \\
\hline Value & 2 & $6(64 \mathrm{QAM})$ & \multirow{2}{*}{1} & \multirow{2}{*}{$948 / 1024$} & $\mu=0$ & 52 & 71.43 \\
\hline & & $8(256 \mathrm{QAM})$ & & $\mu=1$ & 24 & 35.71 \\
\hline
\end{tabular}

\subsubsection{Overheads}

In addition to data packets, the NR SL also carries other necessary channels and signals for operations. These channels and signals count towards transmission Overhead $(\mathrm{OH})$, and is 
reflected in Eq. (18) as overhead ratio $\mathrm{OH}$. The OH in NR SL is composed of the following items:

- PSFCH,

- CSI-RS,

- PT-RS,

- Reserved slots,

- $\mathrm{PSCCH}$,

- 2nd-stage SCI on PSSCH,

- DM-RS for PSSCH,

- AGC symbol,

- Guard symbol, and

- S-SSB.

The ratio $\mathrm{OH}$ is determined by the number of REs occupied by these $\mathrm{OH}$ items, denoted by $\mathrm{RE}_{\mathrm{OH} \text {,slot }}^{\mu}$, over the total number of REs allocated for NR SL, denoted by $\mathrm{RE}_{\mathrm{slot}}^{\mu}$. That is:

$$
O H=\frac{\mathrm{RE}_{\mathrm{OH}, \mathrm{slot}}^{\mu}}{\mathrm{RE}_{\mathrm{slot}}^{\mu}} .
$$

Thus, to achieve maximum capacity in Eq. (18), the resource allocation for NR SL shall be maximized at the target band, and $\mathrm{OH}$ shall be minimized.

Regarding the total number of REs allocated to the SL, as shown in Table 6, the maximum number of PRBs at Band $\mathrm{n} 14$, with a $10 \mathrm{MHz}$ bandwidth, is 52 for $\mu=0$ and 24 for $\mu=1$. This gives the maximum resource allocation of $8736 \mathrm{REs}$ per slot for $\mu=0$ and 4032 REs per slot for $\mu=1$.

Regarding resources occupied by the $\mathrm{OH}$ items, $\mathrm{OH}$ resource allocation can be optimized in a context where each of the $\mathrm{OH}$ items occupies its minimum allowable resource. Towards this goal, we examine each $\mathrm{OH}$ item as shown below.

PSFCH, CSI-RS, PT-RS and Reserved Slots: in NR SL, PSFCH and CSI-RS can be disabled in the RRC, by configuring SL-PSFCH-Config-rl6 to slO and sl-X-Overhead to $n O$, respectively (6.3.5 in [7]). In addition, as the transmission of the PT-RS is only supported in FR2 [12, Section 8.2.3], for Band n14 under study, it is also excluded from $\mathrm{OH}$. That is, the minimum resources occupied by these three items is zero. Fig. 12 illustrates a resulting slot structure with zero occupancy of these three items.

Reserved slots are discussed in Section 3.3. Its period is related to a series of parameters: numerology, S-SSB repetition, number of non-sidelink slots, and sl-TimeResource-r16 
bitmap length [12, Section 8]. By configuring these parameters, the period of the reserved slots can be high enough that the resulting overhead is negligible. Therefore, its impact is excluded from our study.

Here we disable the PSFCH in order to minimize $\mathrm{OH}$ and maximize data rate. This maps to no feedback-based HARQ. In practice when feedback-based HARQ is requested, the PSFCH is enabled. Accordingly, in the slots that the HARQ requests are placed in, three OFDM symbols that were supposed to be allocated for the PSSCH are instead allocated for the PSFCH, its AGC symbol before it, and the guard symbol that follows. A similar configuration is illustrated in Fig. 4 in Section 4.3. By comparing Fig. 4 with Fig. 12, we can see a significant increase of $\mathrm{OH}$ resource allocation, which would lead to a higher $\mathrm{OH}$ ratio and eventually a much lower throughput. We concentrate on capacity in this study and will address this case in our future work.

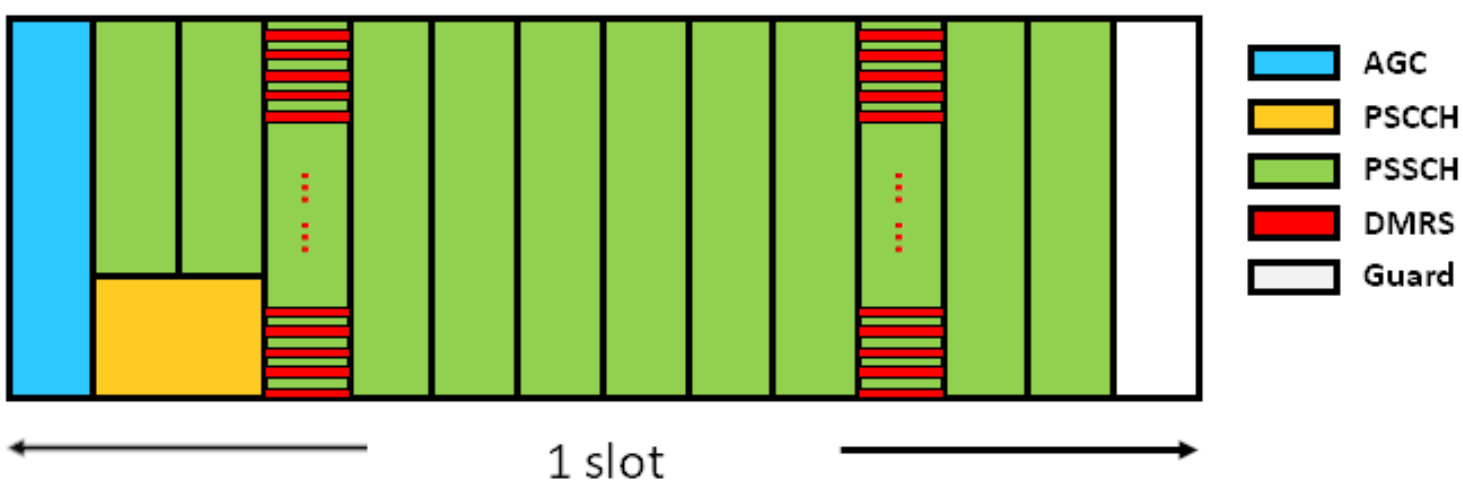

Fig. 12. A slot structure configuration with minimal $\mathrm{OH}$.

PSCCH: The PSCCH carries the 1st-stage SCI. Its resources are allocated at the RRC [7, Section 6.3.5], with related configurations listed as:

- sl-TimeResourcePSCCH-r16: n2, n3,

- sl-FreqResourcePSCCH-r16: n10, n12, n15, n20, n25.

It shows that by configuring sl-TimeResourcePSCCH-rl6 and sl-FreqResourcePSCCH-rl6 in SL-PSCCH-Config-r16, two or three OFDM symbols can be allocated to the PSCCH in the time domain, and 10,12, 15, 20, or $25 \mathrm{RBs}$ can be allocated in the frequency domain, respectively. Therefore, a minimum of $240 \mathrm{REs}$ can be allocated to the PSCCH.

2nd-Stage SCI: The 2nd-stage SCI is carried on the PSSCH. Its number of bits is determined by the 2nd-stage SCI formats, which are: 35 bits for 2-A, and 48 bits for 2-B [11, Section 8.4.1.1]. These SCI bits are then attached with 24 CRC bits [11, Section 7.3.2]. After performing channel coding and rate matching on this concatenated bit sequence, the coded bits are modulated with QPSK and multiplexed with the coded bits for the SL-SCH [11, Sections 8.4.3, 8.4.4, 8.4.5, and 8.2]. These procedures jointly determine the number of REs, which is expressed in Eq. (21) [11, Section 8.4.4]: 


$$
Q_{\mathrm{SCI} 2}^{\prime}=\min \left\{\left\lceil\frac{\left(O_{\mathrm{SCI} 2}+L_{\mathrm{SCI} 2}\right) \cdot \beta_{\mathrm{offset}}^{\mathrm{SCI} 2}}{Q_{\mathrm{m}}^{\mathrm{SCI} 2} \cdot R}\right\rceil,\left\lceil\alpha \sum_{l=0}^{N_{\mathrm{symbol}}^{\mathrm{PSSCH}}-1} M_{\mathrm{SC}}^{\mathrm{SCI} 2}(l)\right\rceil\right\}+\gamma,
$$

where the first parameter in min() is the number of REs required for the 2nd-stage SCI after QPSK, and the second parameter is the total number of available REs in the PSSCH, which is the greater one in our context; $O_{\mathrm{SCI} 2}=35$ is the number of bits for the 2nd-stage SCI format 2-A; $L_{\mathrm{SCI} 2}=24$ is the number of CRC bits; $Q_{\mathrm{m}}^{\mathrm{SCI} 2}=2$ is the modulation order of QPSK for the 2nd-stage SCI; $R$ is the target coding rate for the PSSCH, which is $948 / 1024$ from the previous analysis; the minimum value of the coefficient $\beta_{\text {offset }}^{\mathrm{SCI}}$ is 1.125 , which are jointly determined by the beta_offset indicator from the 1st-stage SCI [11, Section 8.3.1.1]), sl-BetaOffsets2ndSCI-rl6 in the RRC [7, Section 6.3.5], and in [10, Table 9.3-2]; and $\gamma$ is the number of vacant REs in the last RB of the 2nd-stage SCI [11, Section 8.4.4].

By plugging all these numbers into Eq. (21), the minimum number of REs required for the 2nd-stage SCI is 36.

$D M-R S$ : The resource occupied by the PSSCH DM-RS can be viewed from the time domain and the frequency domain. In the time domain, resource allocation for the PSSCH DM-RS consists of the number of OFDM symbols occupied and their positions in a slot. The number of OFDM symbols is associated with the time pattern value, which is configured by sl-PSSCH-DMRS-TimePatternList-r16 in the RRC [7, Section 6.3.5] as:

- sl-PSSCH-DMRS-TimePatternList-r16: SEQUENCE (SIZE (1..3)) OF INTEGER 2..4.

It is then interpreted as the number of DM-RS symbols at the PHY. It also indicates that the minimum number of PSSCH DM-RS symbols that can be configured is two.

Following the number of OFDM symbols occupied in a slot, Table 8 indicates the positions of the DM-RS symbols in a slot. The positions are jointly determined by 1) the number of PSCCH symbols, which is configured to 2 in our context, and 2) the number of OFDM symbols for the PSCCH and PSSCH, $l_{\mathrm{d}}$, including the AGC symbol [9, Section 8.4.1.1.2]. As the maximum value of $l_{\mathrm{d}}$ is 13, the two DM-RS symbols are positioned at Symbols 3 and 10, which are illustrated in Fig. 12.

Meanwhile, in the frequency domain, resource allocation for the PSSCH DM-RS follows Configuration Type 1 (8.4.1.1.2 in [9]), in which each two adjacent REs for the PSSCH DM-RS are spaced by an RE for PSSCH data or the 2nd-stage SCI. Mathematically, this relation is expressed as in [9, Section 6.4.1.1.3]:

$$
\begin{aligned}
\tilde{a}_{k, l}^{\left(\tilde{p}_{j, \mu}\right)} & =w_{f}\left(k^{\prime}\right) w_{t}\left(l^{\prime}\right) r\left(2 n+k^{\prime}\right), \\
k & =4 n+2 k^{\prime}+\Delta, \\
k^{\prime} & =0,1, \\
n & =0,1, \ldots,
\end{aligned}
$$


Table 8. PSSCH DM-RS Time-domain Location [9, Table 8.4.1.1.2-1].

\begin{tabular}{|c|c|c|c|c|c|c|}
\hline \multirow{4}{*}{$\begin{array}{c}l_{\mathrm{d}} \text { in } \\
\text { symbols }\end{array}$} & \multicolumn{6}{|c|}{ DM-RS position $\bar{l}$} \\
\hline & \multirow{2}{*}{\multicolumn{3}{|c|}{$\begin{array}{l}\text { PSCCH duration } 2 \text { symbols } \\
\text { Number of PSSCH DM-RS }\end{array}$}} & \multirow{2}{*}{\multicolumn{3}{|c|}{$\begin{array}{l}\text { PSCCH duration } 3 \text { symbols } \\
\text { Number of PSSCH DM-RS }\end{array}$}} \\
\hline & & & & & & \\
\hline & 2 & 3 & 4 & 2 & 3 & 4 \\
\hline 6 & 1,5 & - & - & 1,5 & - & - \\
\hline 7 & 1,5 & - & - & 1,5 & - & - \\
\hline 8 & 1,5 & - & - & 1,5 & - & - \\
\hline 9 & 3,8 & $1,4,7$ & - & 4,8 & $1,4,7$ & - \\
\hline 10 & 3,8 & $1,4,7$ & - & 4,8 & $1,4,7$ & - \\
\hline 11 & 3,10 & $1,5,9$ & $1,4,7,10$ & 4,10 & $1,5,9$ & $1,4,7,10$ \\
\hline 12 & 3,10 & $1,5,9$ & $1,4,7,10$ & 4,10 & $1,5,9$ & $1,4,7,10$ \\
\hline 13 & 3,10 & $1,6,11$ & $1,4,7,10$ & 4,10 & $1,6,11$ & $1,4,7,10$ \\
\hline
\end{tabular}

where $k$ is the RE index. Since $\Delta=0$ (in [9, Table 6.4.1.1.3-1]), then $k=0,2,4,8, \ldots$, which are for all even RE indexes. Therefore, the number of REs per RB for a PSSCH DM-RS is six in each DM-RS symbol.

By combining the time domain of two DM-RS symbols per slot and the frequency domain of six REs per RB in each DM-RS symbol, and noting the total number of RBs for different numerologies in Table 6, the total number of REs for the PSSCH DM-RS is 624 for $\mu=0$ and 288 for $\mu=1$. Note that the resulting number of REs occupied by the DM-RS is a function of channel bandwidth as well as the numerology employed. That is, the $O H$ in Eq. (18) is not independent of channel bandwidth and numerology, or $N_{P R B}^{B W, \mu}$ and $T_{S}^{\mu}$.

AGC and Guard Symbols: NR SL employs AGCs and guard symbols. The first symbol in each slot is the duplication of the first multiplexed symbol of the PSCCH and PSSCH [9, Sections 8.3.1.5 and 8.3.2.3]. This duplicated symbol serves as an AGC symbol. Meanwhile, in case the PSFCH is enabled, its first mapped OFDM symbol, including the DMRS, PT-RS, or CSI-RS, is duplicated in the immediate preceding symbol. This duplicated symbol serves as the AGC symbol for the PSFCH [9, Section 8.3.4.2.2]. Additionally, a guard symbol follows the last symbol of the PSSCH, PSFCH, or S-SSB [9, Section 8.2.1]. As mentioned previously, in this study the PSFCH is not enabled. Consequently, one AGC and one guard symbol are allocated in each slot, as illustrated in Fig. 12, and the number of REs for each of them are 624 when $\mu=0$ and 288 when $\mu=1$.

$S$-SSB: The S-SSB carries the PSBCH and Sidelink Primary/Secondary Synchronization Signals. The transmission period of the S-SSB is 16 frames, and the number of S-SSBs within a period can be configured by sl-NumSSB-WithinPeriod-rl6 with a minimum value of 1 [7, Section 6.3.5]. That is, the S-SSB can be transmitted at a minimum rate of once per 16 frames. Mapping this rate to slots under different numerologies leads to once every 160 slots for $\mu=0$ and once every 320 slots for $\mu=1$, respectively [9, Section 4.3.2].

As the set of slots for the resource pool, i.e., for transmission or reception of the PSSCH, 
does not include the slots for the S-SSB [12, Section 8], the un-allocated resources in the slots that contain S-SSBs are not intended for the PSSCH. Therefore, the overhead due to $\mathrm{S}-\mathrm{SSB}$ comes mainly from its occupancy in the time domain. In the time domain, each S-SSB occupies 13 OFDM symbols (under normal cyclic prefix) plus one guard symbol [9, Section 8.4.3.1].

By combining the S-SSB rate and the size of each slot that contains an S-SSB, the OH due to S-SSBs can be averaged to the slot level as

$$
\mathrm{RE}_{\mathrm{OH}, \mathrm{S}-\mathrm{SSB}}^{\mu=0}=\frac{(13+1) \times 52 \times 12}{160}=54.6 \mathrm{REs} / \mathrm{slot}
$$

for $\mu=0$, and

$$
\mathrm{RE}_{\mathrm{OH}, \mathrm{S}-\mathrm{SSB}}^{\mu=1}=\frac{(13+1) \times 24 \times 12}{320}=12.6 \mathrm{REs} / \mathrm{slot}
$$

for $\mu=1$.

In summary, Table 9 lists the number of REs each $\mathrm{OH}$ item occupied per slot, in Columns 2 and 3. The total number of REs occupied by all items is calculated in the last row. In addition, the percentage of each $\mathrm{OH}$ item over the total $\mathrm{OH}$ is listed in Columns 3 and 4. It shows that $\mathrm{OH}$ mainly comes from three items-DM-RS, AGC, and guard symbols - with each contributing almost $30 \%$ and in total around $85 \%$ of the overall $\mathrm{OH}$. Following the three is the PSCCH, which contributes around $10 \%$. The contribution of other items, i.e., 2nd-stage SCI (labeled as SCI2 on the table) and S-SSB, is minimal.

The last two columns of Table 9 shows the overhead over total number of REs, both from each $\mathrm{OH}$ item and their sum, which maps to the $\mathrm{OH}$ term in Eq. (18). It shows that the $\mathrm{OH}$ occupies around $1 / 4$ of the overall resources. Note that while numerology 1 uses a larger SCS and supports more slots, it also introduces $\sim 3 \%$ higher $\mathrm{OH}$ compared with numerology 0. This is because a larger SCS leads to a lower number of total REs per slot. For the $\mathrm{OH}$ item DM-RSs, AGCs, guard symbols and S-SSBs do not contribute to the change of the overhead ratio since they scale with the total number of REs. However, for the PSCCH and 2nd-stage SCI, their sizes stay the same, which results in an increased overhead ratio when the total number of REs is lower. Later these total $\mathrm{OH}$ ratios will be substituted into Eq. (18) for a capacity calculation.

\subsubsection{Blind Transmissions}

As mentioned previously, one major difference between NR SL and LTE SL is that NR supports a configurable HARQ while LTE uses blind transmissions only. The configurable HARQ in NR allows for efficient use of bandwidth by eliminating unnecessary retransmissions. It works especially well in capacity under study, as capacity is achieved when the channel quality is good and no retransmissions are necessary. Nevertheless, to adapt to different scenarios, NR also supports the configuration of blind transmissions, with up to 32 total transmissions (including both the initial transmission and retransmissions). In this section we consider capacity under this configuration. 
Table 9. Resource Allocation of OH Items

\begin{tabular}{|l|c|c|c|c|c|c|}
\hline & \multicolumn{2}{|c|}{ REs/Slot } & \multicolumn{2}{c|}{ REs/Total OH REs } & \multicolumn{2}{c|}{ REs/Total REs } \\
& $\mu=0$ & $\mu=1$ & $\mu=0$ & $\mu=1$ & $\mu=0$ & $\mu=1$ \\
\hline PSFCH & 0 & 0 & 0 & 0 & 0 & 0 \\
\hline CSI-RS & 0 & 0 & 0 & 0 & 0 & 0 \\
\hline PT-RS & 0 & 0 & 0 & 0 & 0 & 0 \\
\hline PSCCH & 240 & 240 & $10.90 \%$ & $20.82 \%$ & $2.75 \%$ & $5.95 \%$ \\
\hline SCI2 & 36 & 36 & $1.63 \%$ & $3.12 \%$ & $0.41 \%$ & $0.89 \%$ \\
\hline DM-RS & 624 & 288 & $28.33 \%$ & $24.99 \%$ & $7.14 \%$ & $7.14 \%$ \\
\hline AGC & 624 & 288 & $28.33 \%$ & $24.99 \%$ & $7.14 \%$ & $7.14 \%$ \\
\hline Guard & 624 & 288 & $28.33 \%$ & $24.99 \%$ & $7.14 \%$ & $7.14 \%$ \\
\hline S-SSB & 54.6 & 12.6 & $2.48 \%$ & $1.09 \%$ & $0.63 \%$ & $0.31 \%$ \\
\hline \hline Total & 2202.6 & 1152.6 & $100 \%$ & $100 \%$ & $25.21 \%$ & $28.59 \%$ \\
\hline
\end{tabular}

When blind transmission is configured, all the transmissions of a TB contain the same information bits. Note that the contribution from the S-SSB to $O H$ is minimal. Hence, to calculate the maximum capacity and in order to reuse Eq. (18) for an approximation, we could take all the retransmissions as overhead in the calculation and include them into $\mathrm{OH}$ in Eq. (18). To do so, since $\mathrm{OH}$ in Eq. (18) is per slot while blind retransmissions can occupy multiple slots, we could map existing $\mathrm{OHs}$, plus the retransmission overhead into one slot by averaging them over the slots for the same TB, as below:

$$
\overline{O H}_{\text {slot }}^{\mu, N}=\frac{\mathrm{RE}_{\mathrm{OH}, \mathrm{slot}}^{\mu}+\mathrm{RE}_{\text {slot }}^{\mu} \cdot(N-1)}{\mathrm{RE}_{\text {slot }}^{\mu} \cdot N},
$$

where $\overline{O H}_{\text {slot }}^{\mu, N}$ is the average overhead ratio including the blind retransmissions, and $N$ is the configured number of transmissions per TB.

Further manipulation of the above equation yields:

$$
1-\overline{O H}_{\text {slot }}^{\mu, N}=\frac{1-O H}{N}
$$

where $O H=\frac{\mathrm{RE}_{\text {OH,slot }}^{\mu}}{\mathrm{RE}_{\text {slot }}^{\mu}}$ as in Eq. (20).

Eq. (26) tells us that the capacity under the blind transmission configuration can be captured by the revised $\mathrm{OH}$. With other parameters unchanged and by noting Eq. (18), we then have 
data rate with blind transmissions

$$
\begin{aligned}
& =10^{-6} \cdot v_{\text {layers }} \cdot Q_{m} \cdot f \cdot R_{\max } \cdot \frac{N_{P R B}^{B W, \mu} \cdot 12}{T_{s}^{\mu}} \cdot \frac{1-O H}{N} \\
& =\frac{\text { data rate without blind transmissions }}{N} .
\end{aligned}
$$

That is, Eq. (27) can be used to calculate the capacity with blind transmissions, which is $N$ times less than the capacity without blind transmissions.

Table 10 provides a few examples of transmission numbers and their relationships to REs/Slot and $\overline{O H}_{\text {slot }}^{\mu, N}$. It is evident that a larger $N$ results in a significantly higher $\overline{O H}_{\text {slot }}^{\mu, N}$. The capacity changes caused by blind transmissions are discussed in the following section.

Table 10. Resource Allocation with Blind Transmissions

\begin{tabular}{|c|c|c|c|c|}
\hline \multirow{2}{*}{$\begin{array}{c}\text { No. of } \\
\text { Transmissions }\end{array}$} & $\mu=0$ & $\mu=1$ & \multicolumn{2}{c|}{ REs/Total REs $\left(\overline{O H}_{\text {slot }}^{\mu, N}\right)$} \\
\hline 1 & 2202.6 & 1152.6 & $25.21 \%$ & $28.59 \%$ \\
\hline \multicolumn{5}{|c|}{$\ldots \ldots$} \\
\hline 4 & 7102.65 & 3312.15 & $81.30 \%$ & $82.15 \%$ \\
\hline \multicolumn{5}{|c|}{$\ldots \ldots$} \\
\hline 32 & 8531.83 & 3942.02 & $97.66 \%$ & $97.77 \%$ \\
\hline
\end{tabular}

\subsubsection{NR Capacity Results}

For NR SL, in the previous two sections, Section 5.3.1 and 5.3.2, we analyzed each parameter and the overhead item required in Eq. (18), and derived capacity formula Eq. (27) for a blind transmission configuration. The resulting numbers were summarized in Tables 7 and 10. By examining the two tables, we can see that different parameter combinations can actually be narrowed down to three independent configurations: numerology, MCS table employed, and HARQ configuration. Categorized by these three dimensions, the resulting NR capacity is listed in Table 11 and also plotted in Fig. 13. Note that for the number of transmissions, three numbers are highlighted for illustration purposes: 1 (equivalent to no retranmissions), 4 (mapping to LTE for comparison purpose) and 32 (maximum NR transmissions per 3GPP).

From Table 11 and Fig. 13 we can read the impacts of the three independent configurations on the resulting capacity. For numerology, we can see that numerology 0 gives a higher capacity number than numerology 1 , and the ratio is fixed to be around $1.14: 1$. The differences come mainly from the term $\frac{N_{P R B}^{B W}, \mu}{T_{s}^{\mu}}$ (Table 6) and $O H$ (Table 9). As discussed in previous sections, numerology 0 has a smaller SCS, which results in both a higher $\frac{N_{P R B}^{B W, \mu}}{T_{s}^{\mu}}$ and a lower $\mathrm{OH}$. 
In addition, depending on the device capabilities, the maximum modulation order one UE supports can be 6 or 8 . This leads to either the 64 QAM or the 256 QAM MCS table being employed without changing the values of other parameters. Consequently, the capacity ratio between the employed two MCS tables is $3: 4$, which is consistent with the numbers in Table 11.

Furthermore, capacity decreases significantly with an increasing number of transmissions. This is consistent with the derived Eq. (27), which shows that capacity follows an inversely proportional trend when compared to the number of transmissions.

Table 11 also shows that the maximum NR SL capacity is around $97 \mathrm{Mbit} / \mathrm{s}$, which, as expected and analyzed above, is achieved under configuration with numerology 0, 256 QAM MCS table, and no HARQ retransmissions. This is significantly higher than the LTE capacity, which is discussed in the next section.

Table 11. NR SL Capacity in Mbit/s

\begin{tabular}{|c|c|c|c|c|}
\hline No. of & \multicolumn{2}{|c|}{ 64 QAM table } & \multicolumn{2}{c|}{ 256 QAM table } \\
Transmissions & $\mu=0$ & $\mu=1$ & $\mu=0$ & $\mu=1$ \\
\hline 1 & 72.58 & 63.98 & $\mathbf{9 6 . 7 8}$ & 85.30 \\
\hline \multicolumn{5}{|c|}{$\ldots \ldots$} \\
\hline 4 & 18.15 & 15.99 & 24.19 & 21.33 \\
\hline \multicolumn{5}{|c|}{$\ldots \ldots$} \\
\hline
\end{tabular}

\subsection{LTE and NR Capacity Comparison}

In Section 5.2 we obtained an LTE capacity of $5.28 \mathrm{Mbit} / \mathrm{s}$. In Section 5.3 we see that NR capacity varies per configuration with a maximum of $96.78 \mathrm{Mbit} / \mathrm{s}$, which is significantly higher than LTE (around 18 times greater) and is achieved with numerology 0, 256 QAM MCS and no retransmissions. Eq. (18) and (27) provide some insights on where the gains come from.

The first improvement results from the use of 2-layer spatial multiplexing in NR versus no MIMO in LTE. This gives NR two times the data rate of LTE. The second is the higher NR MCS. NR can use 256 QAM with a 948/1024 coding rate, compared to 16 QAM with lower coding rate in LTE. The most significant NR improvement comes from the configurable HARQ. Considering the best NR case with no retransmissions versus LTE , which requires four transmissions, this gives NR approximately four times the achievable data in LTE (Eq. (27)). Added together, these three factors contribute to approximately 18 times the data rate of LTE.

Another perspective is that in order to adapt to different deployment scenarios and device capabilities, NR supports various configurations with different capacity numbers as described in Section 5.3. As shown in Fig. 13, the factors discussed above still work for various configurations except with blind retransmissions. As shown, the NR improvement 


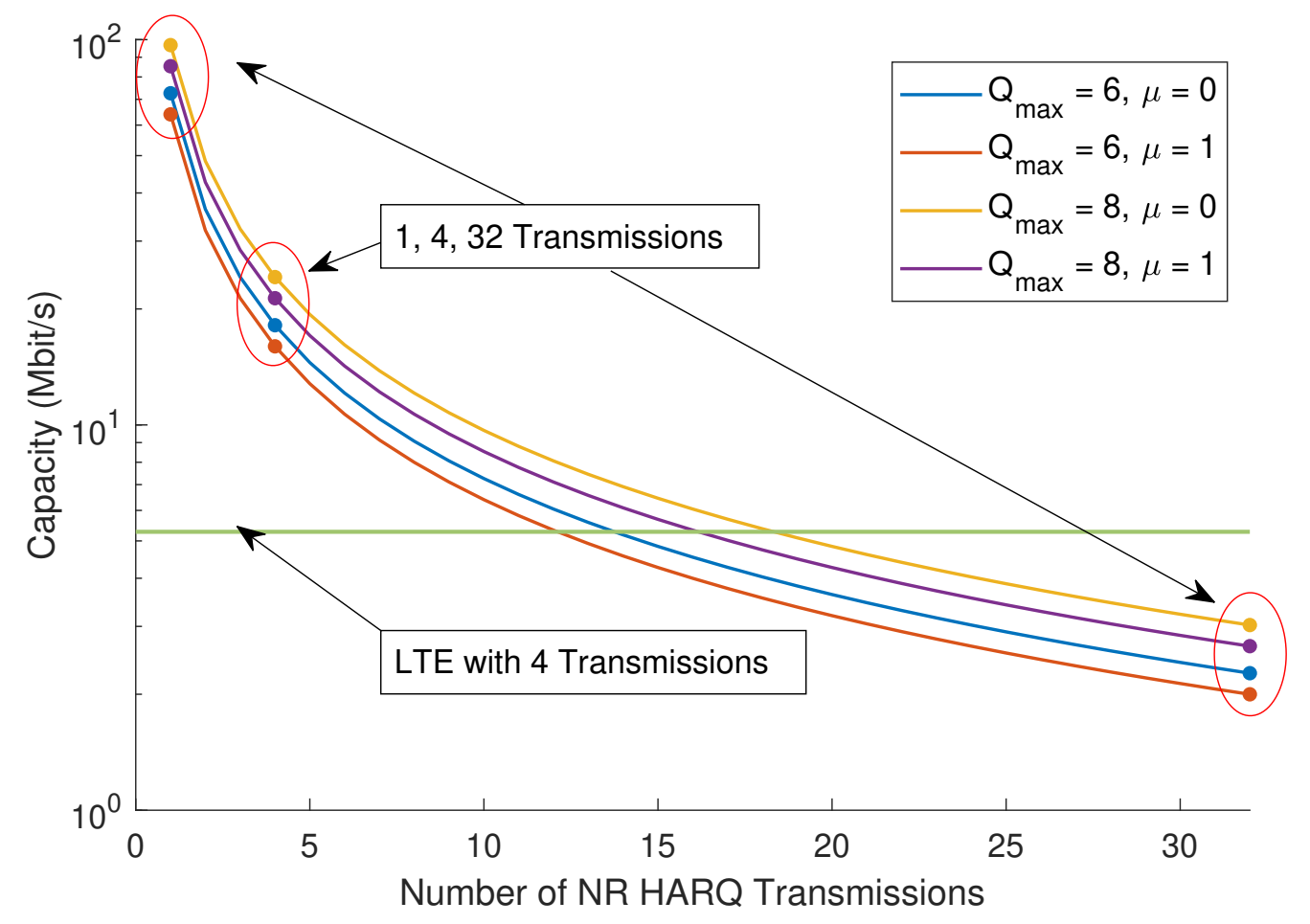

Fig. 13. Capacity - NR vs LTE.

decreases significantly with an increasing number of transmissions, and eventually reaches zero when the number is close to $12,14,16$, and 18 respectively. NR capacity then falls below LTE capacity thereafter. The configuration with such a high number of transmissions is typically used when ultra reliability is required, and high capacity is less critical.

While NR has much higher capacity numbers in general, the numbers map to the maximum data rate achievable. In practice, the data may be much lower due to various factors. One example is the significantly higher $\mathrm{OH}$ caused by enabling the PSFCH when feedbackbased HARQ is configured (Section 5.3.2), which would eventually lead to a much lower data rate.

\subsection{Summary}

In this section, we analyzed LTE SL and NR SL capacity under Band 14 / Band n14 $10 \mathrm{MHz}$ bandwidth and with a focus on unicast. Our initial quantitative results show that NR's new features improve capacity significantly, except when blind transmissions are configured with a high number of transmissions. The improvement varies by configurations and can be as high as around 18 times, which can be achieved with the highest configuration, specifically, 256 QAM MCS table with numerology 0 and no HARQ retransmissions. Each of the new features contributes to a portion of the improvement. Using the highest configuration as an example, up to two-layer spatial multiplexing contributes two times, the higher order 
of MCS contributes to slightly above two times, and the most improvement comes from the supported configurable HARQ - by eliminating the four blind transmissions employed by LTE, the NR capacity is improved by four times.

Note that NR allows flexible configurations to adapt to different deployment scenarios and device capabilities, hence different capacity numbers are achieved as a balance to meet service requirements. One extreme case is the configuration with a high number of blind transmissions. While NR could have less capacity than LTE, this configuration is useful when ultra reliability is required and a high capacity is relatively not so critical. Also note that the high NR capacity in general maps to the highest data rate achievable. The actual data rate could be lower due to various factors such as significantly higher overhead due to the configured feedback channel.

While our initial capacity study focuses on unicast, one next step is to evaluate capacity for multicast and broadcast together with related major factors.

\section{Quality of Service}

\subsection{Introduction}

5G NR inherits LTE's framework of handling different QoS requirements, and enhances it in several aspects.

Over the core network and Uu interface, NR's QoS handling principles are similar to LTE's: the core network specifies QoS requirements, and the radio-access network maps and handles packets accordingly. The exact mechanisms and terms are somewhat different between NR and LTE though. Between the NR network and the connected device, there is one or more Protocol Data Unit (PDU) sessions, which correspond to one or more QoS flows and DRBs. A general overview of the NR QoS model is provided in Section 6.2 first, to better understand and compare with the QoS operations over NR SL described in the following sections. Unlike LTE, the mapping between QoS flows to DRBs are done by a separate layer in the Radio Access Network (RAN), namely, the SDAP layer. A brief introduction of the SDAP layer is given in Section 6.3, including SDAP's support for NR SL QoS handling.

The QoS support for direct mode communication over SL has been improved considerably in NR. Section 6.4 describes the NR SL QoS handling in a nutshell, and points out the correspondence between LTE ProSe QoS parameters and NR SL QoS parameters. Section 6.5 focuses on the new QoS parameter, Range, that NR provided for direct mode communication. Although the majority of the standardized NR SL QoS mechanisms so far are provisions for V2X scenarios, they may be applicable to public safety use cases as well. In addition, there are ongoing 3GPP discussions on QoS support designed for public safety applications specifically, as listed in Section 6.6. 


\subsection{Overview of New Radio Quality of Service Model}

Fig. 14 illustrates NR's overall QoS architecture briefly. 5G Core Network (5GC) establishes one or more PDU sessions for each UE. There may be one or multiple QoS flows within each PDU session. The Next-Generation Radio Access Network (NG-RAN) may map one or multiple QoS flows to the same DRB. 5GC and NG-RAN map packets in both directions with proper QoS flows and DRBs to satisfy QoS requirements. Table 12 outlines UL and DL operations for mapping packets from IP-flows to QoS flows and then from QoS flows to DRBs. The QoS Flow Identifier (QFI) mentioned in the table is embedded in the header over the user plane interface (NG-U) between NG-RAN and 5GC. It identifies a QoS Flow within a PDU session, and its value may be dynamically assigned or may be equal to the $5 \mathrm{G}$ QoS Identifier (5QI), a QoS parameter which will be discussed later. The reflective mapping in the UL is a new function introduced in NR. When it is enabled, a UE monitors the QoS flow to DRB mapping rule in the DL, and applies it in the UL accordingly.

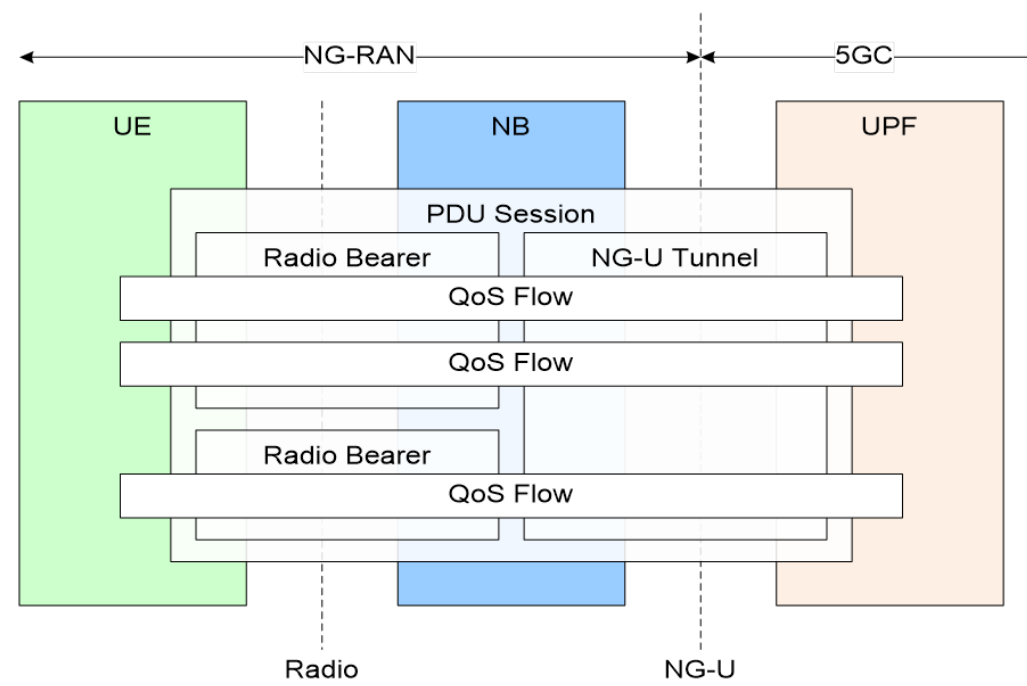

Fig. 14. QoS architecture [22, Fig. 12-1]. ${ }^{2}$

${ }^{2} \odot 2020$ - 3GPPTM deliverables and material are the property of Association of Radio Industries and Businesses (ARIB), Alliance for Telecommunications Industry Solutions (ATIS), China Communications Standards Association (CCSA), European Telecommunications Standards Institute (ETSI), Telecommunications Standards Development Society, India (TSDSI), Telecommunications Technology Association (TTA) and Telecommunications Technology Committee (TTC) who jointly own the copyright in them. They may be subject to further modifications and are therefore provided to you "as is" for information purposes only. Further use is strictly prohibited. 


\begin{tabular}{|l|l|l|}
\hline & \multicolumn{1}{|c|}{ DL } & UL \\
\hline Characterization of QoS flows @ NAS level & $\begin{array}{l}\text { QoS profiles } \\
\text { (provided by 5GC to NG-RAN) }\end{array}$ & $\begin{array}{l}\text { QoS rules } \\
\text { (provided by 5GC to the UE) }\end{array}$ \\
\hline $\begin{array}{l}\text { From IP-flows to QoS flows: } \\
\text { Non-Access Stratum (NAS) level mapping }\end{array}$ & Done by NAS level packet filter @5GC & Done by NAS level packet filter @ UE \\
\hline $\begin{array}{l}\text { From QoS Flows to DRBs: } \\
\text { AS level mapping }\end{array}$ & $\begin{array}{l}\text { Follow AS level mapping rules @ NG-RAN. } \\
\text { Based on QoS Flow Identifier (QFI) and } \\
\text { the associated QoS profiles. }\end{array}$ & $\begin{array}{l}\text { Follow AS level mapping rules which are } \\
\text { signaled to UE either explicitly (RRC) or } \\
\text { implicitly (reflective mapping) }\end{array}$ \\
\hline
\end{tabular}

Table 12. NR QoS Mapping Operations

As listed in Table 12, NG-RAN uses the QoS profile to determine how to handle packets over the radio interface according to QoS requirements. The QoS profile of a QoS flow contains QoS parameters, such as 5QI and Allocation and Retention Priority (ARP). In addition, depending on whether the QoS flow is a Guaranteed Bit Rate (GBR) flow or not, more QoS parameters may be included in the QoS profile. For a Non-GBR QoS flow, the Reflective QoS Attribute (RQA) parameter and other additional QoS Flow Information may be included. For a GBR QoS flow, the following QoS parameters may be specified [23]:

- Guaranteed Flow Bit Rate (GFBR) for both UL and DL;

- Maximum Flow Bit Rate (MFBR) for both UL and DL;

- Maximum Packet Loss Rate for both UL and DL;

- Delay Critical Resource Type;

- Notification Control.

Among the above QoS parameters, the 5QI is associated to QoS characteristics such as priority level, Packet Delay Budget (PDB), Packet Error Rate (PER), averaging window, and maximum data burst volume. If the 5QI value in a QoS profile is a standardized or pre-configured one, such as those listed in Table 13, the 5G QoS characteristics of that QoS flow can be derived from the 5QI value and do not need to be explicitly signaled. Otherwise, QoS characteristics will be signaled as part of the QoS profile of that QoS flow.

Besides the above QoS requirements, which specify per QoS flow expectations, a few other parameters may also be configured to guide/limit the interaction and coexistence among different flows and/or sessions. For example, the Session-Aggregate Maximum Bit Rate (AMBR) limits the aggregate bit rate that can be provided across all Non-GBR QoS Flows for a specific PDU Session. The UE-AMBR limits the aggregate bit rate that can be expected across all Non-GBR QoS Flows of a UE, and it is guaranteed by the RAN. When the transmission demand from various flows cannot be met simultaneously, prioritization will be done based on QoS information.

When comparing 5QI defined for NR ([23] Table 5.7.4-1) with QoS Class Identifier (QCI) defined for LTE ([24] Table 6.7.1-A), we can see that new values 82, 83, 84, 85, and 86 are introduced in NR, among which 83,85 , and 86 are recommended for V2X 


\begin{tabular}{|c|c|c|c|c|c|c|c|}
\hline $\begin{array}{c}5 \mathrm{SQI} \\
\text { Value }\end{array}$ & $\begin{array}{c}\text { Resource } \\
\text { Type }\end{array}$ & $\begin{array}{c}\text { Default } \\
\text { Priority } \\
\text { Level } \\
\end{array}$ & $\begin{array}{l}\text { Packet } \\
\text { Delay } \\
\text { Budget }\end{array}$ & $\begin{array}{l}\text { Packet } \\
\text { Error } \\
\text { Rate } \\
\end{array}$ & $\begin{array}{c}\text { Default } \\
\text { Maximum Data } \\
\text { Burst Volume } \\
\end{array}$ & $\begin{array}{c}\text { Default } \\
\text { Averaging } \\
\text { Window } \\
\end{array}$ & Example Services \\
\hline 3 & \multirow[t]{3}{*}{ GBR } & 30 & $50 \mathrm{~ms}$ & $10^{-3}$ & $\mathrm{~N} / \mathrm{A}$ & $2000 \mathrm{~ms}$ & $\begin{array}{l}\text { Real Time Gaming, V2X messages } \\
\text { (see TS 23.287). } \\
\text { Electricity distribution - medium } \\
\text { voltage, Process automation } \\
\text { monitoring }\end{array}$ \\
\hline 65 & & 7 & $75 \mathrm{~ms}$ & $10^{-2}$ & $\mathrm{~N} / \mathrm{A}$ & $2000 \mathrm{~ms}$ & $\begin{array}{l}\text { Mission Critical user plane Push To } \\
\text { Talk voice (e.g., MCPTT) }\end{array}$ \\
\hline 67 & & 15 & $100 \mathrm{~ms}$ & $10^{-3}$ & $\mathrm{~N} / \mathrm{A}$ & $2000 \mathrm{~ms}$ & Mission Critical Video user plane \\
\hline 69 & \multirow[t]{3}{*}{ Non-GBR } & 5 & $60 \mathrm{~ms}$ & $10^{-6}$ & $\mathrm{~N} / \mathrm{A}$ & $\mathrm{N} / \mathrm{A}$ & $\begin{array}{l}\text { Mission Critical delay sensitive } \\
\text { signalling (e.g., MC-PTT signalling) }\end{array}$ \\
\hline 70 & & 55 & $200 \mathrm{~ms}$ & $10^{-6}$ & $\mathrm{~N} / \mathrm{A}$ & $\mathrm{N} / \mathrm{A}$ & $\begin{array}{l}\text { Mission Critical Data (e.g. example } \\
\text { services are the same as } 5 \mathrm{QI} \\
6 / 8 / 9)\end{array}$ \\
\hline 79 & & 65 & $50 \mathrm{~ms}$ & $10^{-2}$ & $\mathrm{~N} / \mathrm{A}$ & $\mathrm{N} / \mathrm{A}$ & V2X messages (see TS 23.287) \\
\hline 83 & \multirow[t]{3}{*}{$\begin{array}{l}\text { Delay } \\
\text { Critical } \\
\text { GBR }\end{array}$} & 22 & $10 \mathrm{~ms}$ & $10^{-4}$ & 1354 bytes & $2000 \mathrm{~ms}$ & $\begin{array}{l}\text { Discrete Automation (see } \\
\text { TS 22.261); } \\
\text { V2X messages (UE - RSU } \\
\text { Platooning, Advanced Driving: } \\
\text { Cooperative Lane Change with low } \\
\text { LoA. See TS 22.186, TS 23.287) }\end{array}$ \\
\hline 85 & & 21 & $5 \mathrm{~ms}$ & $10^{-5}$ & 255 bytes & $2000 \mathrm{~ms}$ & $\begin{array}{l}\text { Electricity Distribution- high voltage } \\
\text { (see TS 22.261). } \\
\text { V2X messages (Remote Driving. } \\
\text { See TS 22.186, TS 23.287) }\end{array}$ \\
\hline 86 & & 18 & $5 \mathrm{~ms}$ & $10^{-4}$ & 1354 bytes & $2000 \mathrm{~ms}$ & $\begin{array}{l}\text { V2X messages (Advanced Driving: } \\
\text { Collision Avoidance, Platooning } \\
\text { with high LoA. See TS 22.186, } \\
\text { TS 23.287) }\end{array}$ \\
\hline
\end{tabular}

Table 13. SL related standardized 5QI to QoS characteristics mapping (selected from [23, Table 5.7.4-1] $)^{3}$.

messages. Also, a couple of additional QoS characteristics are indicated by 5QI for NR, namely, "Maximum Data Burst Volume" and "Averaging Window".

\subsection{SDAP Layer for Quality of Service}

The SDAP layer is a new layer in the user plane, responsible for mapping QoS bearers to radio bearers based on QoS requirements. It is added above the Packet Data Convergence Protocol (PDCP) layer, and is specified by 3GPP standard TS 37.324 [25]. The main functions of SDAP layers are [22]:

- transfer of user plane data;

- mapping between a QoS flow and a DRB for both DL and UL;

- mapping between a PC5 QoS flow and an SL-DRB for NR SL communication;

${ }^{3} \mathrm{O} 2020$ - 3GPPTM deliverables and material are the property of ARIB, ATIS, CCSA, ETSI, TSDSI, TTA and TTC who jointly own the copyright in them. They may be subject to further modifications and are therefore provided to you "as is" for information purposes only. Further use is strictly prohibited. 
- marking QFI in both DL and UL packets;

- marking PC5 QoS Flow Identifier (PFI) in unicast mode of NR SL communication packets;

- reflective QoS flow to DRB mapping for the UL SDAP data PDUs.

The above functions can be categorized roughly into two types: 1) mapping functions and 2) data marking and transfer functions.

Among SDAP mapping functions, the reflective QoS flow to DRB mapping is a new function introduced in NR. However, for NR SL communication, reflective PC5 QoS flow to SL-DRB mapping is not supported. Regarding other mapping functions, as illustrated in Fig. 15, a single SDAP entity is configured for each PDU session. Multiple-to-one mapping is allowed between QoS flows and DRBs, while one QoS flow is mapped onto only one DRB at a time in the UL. The mapping rules are configured in the device using RRC signaling. For NR SL communications, similarly, there is a one-to-one mapping between an SDAP entity and each Destination Layer-2 ID and cast type in the UE. Multiple-to-one mapping is allowed between PC5 QoS flows and SL-DRBs, while one PC5 QoS flow is mapped onto only one SL-DRB at a time for the SL transmission.

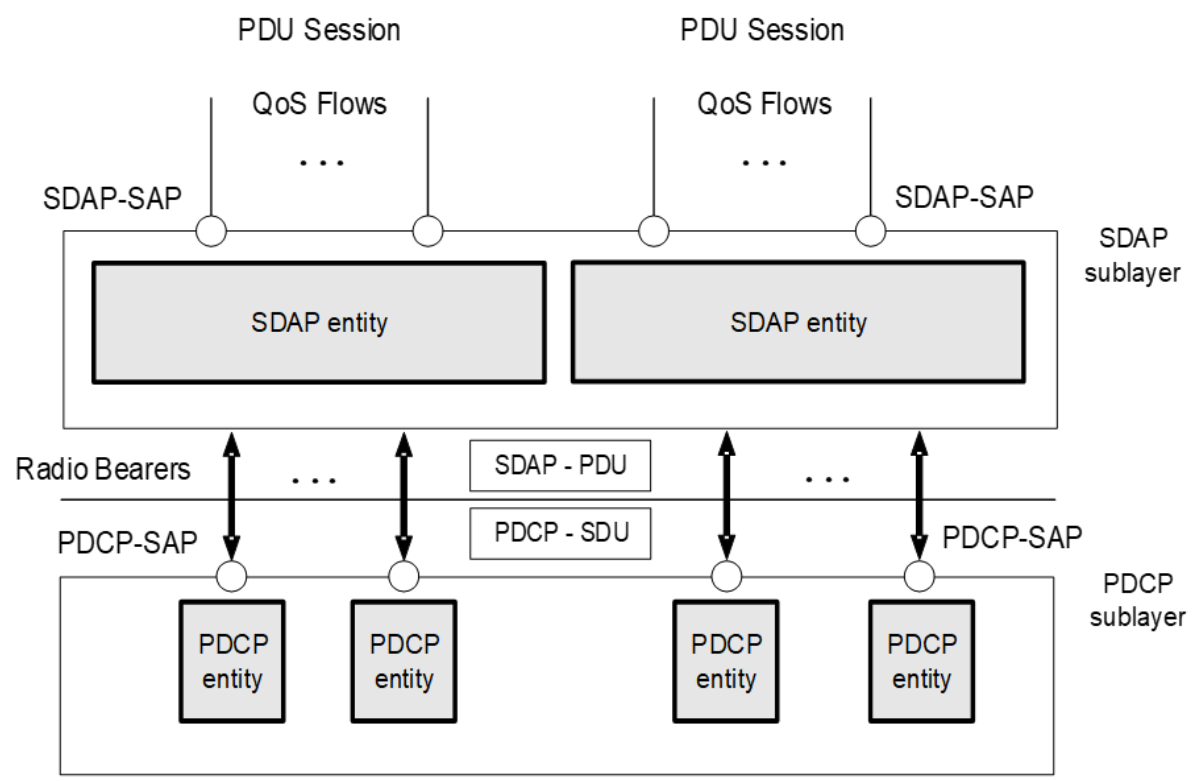

Fig. 15. SDAP sublayer structure view [25, Fig. 4.2.1-1 $]^{4}$.

The SDAP data marking and transfer functions are supported by five data PDU formats and one control PDU format defined in [25], among which, three PDU formats are relevant

${ }^{4} \odot 2020$ - 3GPPTM deliverables and material are the property of ARIB, ATIS, CCSA, ETSI, TSDSI, TTA and TTC who jointly own the copyright in them. They may be subject to further modifications and are therefore provided to you "as is" for information purposes only. Further use is strictly prohibited. 
to NR SL communications. Groupcast and broadcast of NR SL communication only support the SDAP data PDU format without an SDAP header ([25, Figure 6.2.2.2-1] ). Unicast SL communication supports the SDAP data PDU format with an SDAP header ([25, Figure 6.2.2.4-1]). UE's SDAP entity uses End-Marker control PDU ([25, Figure 6.2.3-1]) to stop the mapping from the SDAP Service Data Unit (SDU) of the QoS flow indicated by the QFI/PFI to the DRB/SL-DRB on which the End-Marker PDU is transmitted.

\subsection{Quality of Service over New Radio Sidelink}

For NR SL communication towards the same destination identified by a Destination Layer2 ID, the PC5 QoS Flow is the finest granularity of QoS differentiation. Each QoS flow is identified by a PFI which is unique within the same destination, and the same traffic forwarding treatment (e.g., scheduling and admission threshold) applies to all User Plane (UP) traffic with the same PFIs. Note that the role of PFIs for NR PC5 communication is quite similar to the role of QFIs for NR communication over the Uu interface. As illustrated in Fig. 16, multiple radio bearers may be established between a pair of source and destination Layer-2 IDs, and each radio bearer may correspond to a different PC5 QoS level. Multiple PC5 QoS Flows may be mapped to the same radio bearer, as determined by the AS layer based on the information provided. For broadcast and groupcast mode communication, the L2 link goes to all UEs in proximity identified by the destination Layer-2 ID. Each PC5 QoS Flow is associated with a PC5 QoS Rule and the following PC5 QoS parameters [26]:

- PC5 5QI (PQI);

- PC5 Flow Bit Rates: for GBR QoS flow only, and may include GFBR and MFBR;

- PC5 Link Aggregated Bit Rates: for unicast links and non-GBR flows, and may include per link AMBR (PC5 LINK-AMBR);

- Range: for groupcast type only;

- Default Values: will be used if the corresponding PC5 QoS parameter is not provided by the upper layer.

Among the PC5 QoS parameters above, the Range parameter is a new and unique QoS parameter introduced for NR SL groupcast, and its definition and usage are further studied in Section 6.5. A PQI is a special 5QI, and there is no overlapping between the values of 5QIs and PQIs. The association between standardized PQI values and PC5 QoS characteristics is shown in Table 14. It is also possible to pre-configure PC5 QoS characteristics and indicate them through the PQI value. In addition, non-standardized PC5 QoS characteristics may override the standardized or pre-configured value of PC5 QoS characteristics with restrictions.

Note that when a UE is out of coverage, pre-configuration may be used for NR SL communication. In the situation when the UE's RRC state changes but the updated SL 


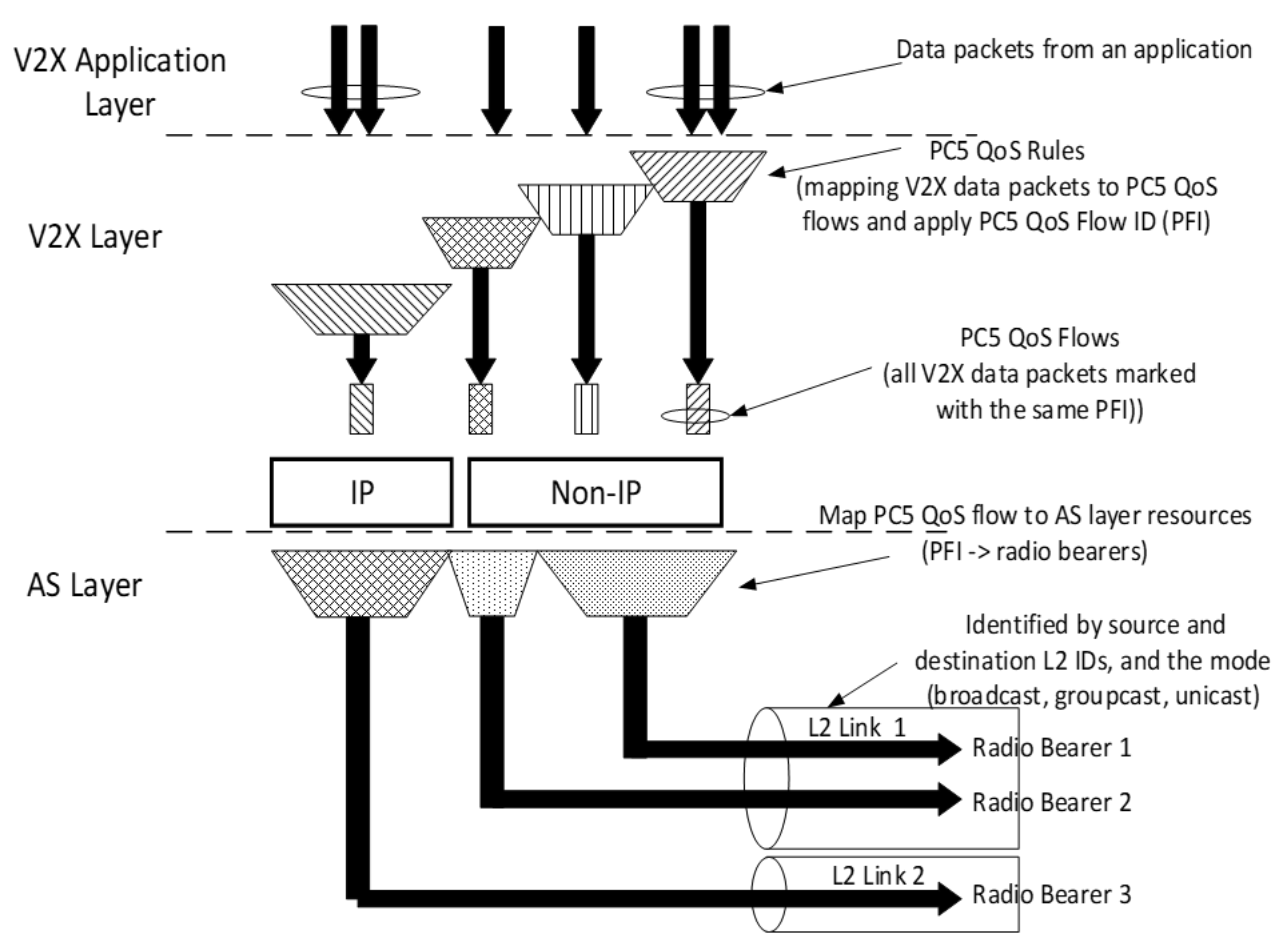

Fig. 16. Handling of PC5 QoS Flows based on PC5 QoS Rules [26, Fig. 5.4.1.1.3-1] $]^{5}$.

DRB configuration(s) hasn't been received for the new RRC state, UE may continue SL data transmissions and receptions using the current configuration of the previous RRC state until getting the configuration for the new RRC state.

For LTE based direct mode communication, i.e., ProSe, the QoS handling is based on ProSe Per-Packet Priority (PPPP) and ProSe Per-Packet Reliability (PPPR) as defined in [27]. For NR based direct mode communication over the PC5 SL, the QoS model is more comprehensive and is quite similar to the QoS model defined for non-SL communication over the Uu interface, with additional parameters such as Range (see Section 6.5). Within the NR SL QoS framework, two QoS characteristics can be viewed as the NR counterpart for the LTE ProSe QoS parameter, PPPP: Priority Level and PDB [26]. NR QoS's Priority Level over NR PC5 has the same format and meaning as LTE PPPP's Priority value over LTE PC5. In addition, NR SL's PDB is derived from the PQI value, e.g., through Table 14, while LTE SL's PPPP value indicates the latency requirement and determines the PDB.

When compared with the NR QoS model over the Uu interface in Section 6.2, it can be seen that the QoS characteristics for SL communication reuse those defined for nonSL communication with minor modifications. In addition, the QoS parameters defined for communications over NR PC5 are similar to those defined for NR Uu in general, with some differentiation, such as the range parameter that is specific to PC5 and the RQA parameter

5 C2020 - 3GPPTM deliverables and material are the property of ARIB, ATIS, CCSA, ETSI, TSDSI, TTA and TTC who jointly own the copyright in them. They may be subject to further modifications and are therefore provided to you "as is" for information purposes only. Further use is strictly prohibited. 


\begin{tabular}{|c|c|c|c|c|c|c|c|}
\hline $\begin{array}{l}\mathrm{PQI} \\
\text { Value }\end{array}$ & $\begin{array}{l}\text { Resource } \\
\text { Type }\end{array}$ & $\begin{array}{c}\text { Default } \\
\text { Priority } \\
\text { Level }\end{array}$ & $\begin{array}{l}\text { Packet } \\
\text { Delay } \\
\text { Budget }\end{array}$ & $\begin{array}{l}\text { Packet } \\
\text { Error } \\
\text { Rate }\end{array}$ & $\begin{array}{l}\text { Default } \\
\text { Maximum } \\
\text { Data Burst } \\
\text { Volume }\end{array}$ & $\begin{array}{c}\text { Default } \\
\text { Averaging } \\
\text { Window }\end{array}$ & Example Services \\
\hline 21 & \multirow{3}{*}{ (NOTE 1) } & 3 & $20 \mathrm{~ms}$ & $10^{-4}$ & $\mathrm{~N} / \mathrm{A}$ & $2000 \mathrm{~ms}$ & $\begin{array}{l}\text { Platooning between UEs - Higher } \\
\text { degree of automation; } \\
\text { Platooning between UE and RSU - } \\
\text { Higher degree of automation }\end{array}$ \\
\hline 22 & & 4 & $50 \mathrm{~ms}$ & $10^{-2}$ & $\mathrm{~N} / \mathrm{A}$ & $2000 \mathrm{~ms}$ & $\begin{array}{l}\text { Sensor sharing - higher degree of } \\
\text { automation }\end{array}$ \\
\hline 23 & & 3 & $100 \mathrm{~ms}$ & $10^{-4}$ & $\mathrm{~N} / \mathrm{A}$ & $2000 \mathrm{~ms}$ & $\begin{array}{l}\text { Information sharing for automated } \\
\text { driving - between UEs or UE and } \\
\text { RSU - higher degree of automation }\end{array}$ \\
\hline 55 & \multirow[t]{5}{*}{ Non-GBR } & 3 & $10 \mathrm{~ms}$ & $10^{-4}$ & $\mathrm{~N} / \mathrm{A}$ & $\mathrm{N} / \mathrm{A}$ & $\begin{array}{l}\text { Cooperative lane change - higher } \\
\text { degree of automation }\end{array}$ \\
\hline 56 & & 6 & $20 \mathrm{~ms}$ & $10^{-1}$ & $\mathrm{~N} / \mathrm{A}$ & $\mathrm{N} / \mathrm{A}$ & $\begin{array}{l}\text { Platooning informative exchange - } \\
\text { low degree of automation; } \\
\text { Platooning - information sharing } \\
\text { with RSU }\end{array}$ \\
\hline 57 & & 5 & $25 \mathrm{~ms}$ & $10^{-1}$ & $\mathrm{~N} / \mathrm{A}$ & $\mathrm{N} / \mathrm{A}$ & $\begin{array}{l}\text { Cooperative lane change - lower } \\
\text { degree of automation }\end{array}$ \\
\hline 58 & & 4 & $100 \mathrm{~ms}$ & $10^{-2}$ & $\mathrm{~N} / \mathrm{A}$ & $\mathrm{N} / \mathrm{A}$ & $\begin{array}{l}\text { Sensor information sharing - lower } \\
\text { degree of automation }\end{array}$ \\
\hline 59 & & 6 & $500 \mathrm{~ms}$ & $10^{-1}$ & $\mathrm{~N} / \mathrm{A}$ & $\mathrm{N} / \mathrm{A}$ & Platooning - reporting to an RSU \\
\hline 90 & \multirow{2}{*}{$\begin{array}{c}\text { Delay } \\
\text { Critical } \\
\text { GBR } \\
\text { (NOTE 1) }\end{array}$} & 3 & $10 \mathrm{~ms}$ & $10^{-4}$ & 2000 bytes & $2000 \mathrm{~ms}$ & $\begin{array}{l}\text { Cooperative collision avoidance; } \\
\text { Sensor sharing - Higher degree of } \\
\text { automation; } \\
\text { Video sharing - higher degree of } \\
\text { automation }\end{array}$ \\
\hline 91 & & 2 & $3 \mathrm{~ms}$ & $10^{-5}$ & 2000 bytes & $2000 \mathrm{~ms}$ & $\begin{array}{l}\text { Emergency trajectory alignment; } \\
\text { Sensor sharing - Higher degree of } \\
\text { automation }\end{array}$ \\
\hline
\end{tabular}

Table 14. Standardized PQI to QoS characteristics mapping [26, Table 5.4.4-1] .

that is specific to the Uu interface.

\subsection{Minimum Communication Range}

A new feature introduced in NR SL group communication is the ability to control the area where the QoS needs to be maintained. As shown in Fig. 17, a transmitting device can specify the communication range requirements associated with a packet transmission. This information is communicated inside the SCI format 2-B control message in the form of an index that maps to a SL pool parameter, which ultimately represents the distance, in meters, where the QoS parameters apply. The UE determines the value to apply based on the longest communication range (defined by the PC5 QoS parameter) of the logical channels in the Medium Access Control (MAC) PDUs to transmit. A user (e.g., RX 1) that is within the communication range will send a NACK message back to the transmitter if there is a decoding error. For devices situated outside the communication range (e.g.,

${ }^{6} \mathrm{C} 2020$ - 3GPPTM deliverables and material are the property of ARIB, ATIS, CCSA, ETSI, TSDSI, TTA and TTC who jointly own the copyright in them. They may be subject to further modifications and are therefore provided to you "as is" for information purposes only. Further use is strictly prohibited. 
RX 2), the communication is best effort, which means that while they may still be able to receive the information, they will not send a NACK message even if there is a decoding error. Since the feature is based on the distance between two devices, the devices' locations are needed to operate properly. In the event that the transmitter does not have its location, the feature cannot be used even if a logical channel was configured to use it. When it is a receiver device (e.g., RX 3) that cannot determine its location, 3GPP has defined a conservative approach and the device assumes that it is within range and will therefore send a NACK if there is a decoding error. The transmitter location is also indicated in the SCI format 2-B using a zone identifier that is derived from its latitude and longitude information. A receiver device then takes the center of the zone as an approximation for the transmitter's location to calculate its distance to the transmitter.

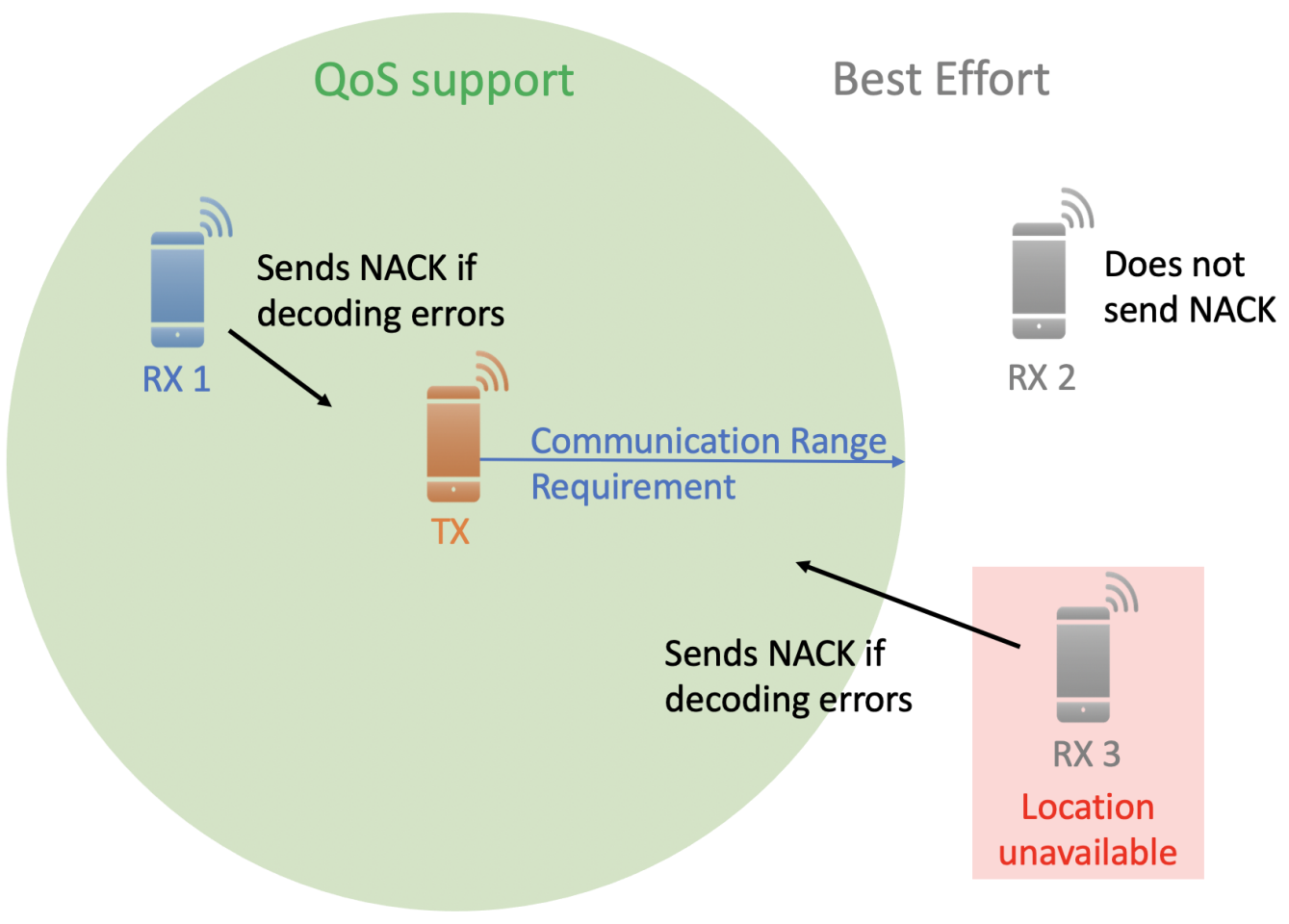

Fig. 17. Overview of Minimum Communication Range (MCR) for group communication.

This new capability enhances the reliability of the communication in group scenarios by allowing retransmissions until all members of the group decode the transmission, up to the maximum number of retransmissions allowed, without adding overhead to create groups. Introduced to support V2X scenarios, it is very useful since vehicles come in and out of range of each other due to traffic patterns and user itineraries. Since first responders heavily rely on voice, group communication, they could certainly benefit from it. However, research is still necessary to understand and optimize the configuration parameters. For example, we need to evaluate the impact of the communication range parameter on the group 
performance. If the communication range is too high (in Release 16, the maximum range is $1000 \mathrm{~m}$ ), it may lead to constant retransmissions and may affect overall performance (e.g., latency) by trying to reach users beyond the incident area. The range is also likely to be dependent on the environment or scenario, with a larger range applicable to open spaces and a shorter range applicable to urban environments.

\subsection{Quality of Service Support for Public Safety}

As defined in Table 13, separate sets of 5QIs have been assigned to mission critical services (i.e., 65, 67, 69, and 70) and V2X messages (i.e., 3, 79, 83, 85, and 86) already, among which, 5QI-65 and 5QI-69, are expected to be used together to provide MCPTT service. Besides the NR QoS handling already in place for general applications, there are ongoing 3GPP standardization efforts to provide more tailored support to public safety services. In addition to existing PQI values defined in Table 14, 3GPP working groups are also discussing the necessity and feasibility of defining new PQI values for public safety services over NR SL [28]. The new PQIs proposed in [28] for public safety services are:

- New value \#1: default priority level 1, PDB $150 \mathrm{~ms}$, and PER 0.01. It may be assigned to services such as mission critical user plane push to talk voice;

- New value \#2: default priority level 2, PDB $200 \mathrm{~ms}$, and PER 0.01. It may be assigned to services such as non-mission-critical user plane push to talk voice;

- New value \#3: default priority level 2, PDB $200 \mathrm{~ms}$, and PER 0.001. It may be assigned to services such as mission critical video user plane;

- New value \#4: default priority level 1, PDB $120 \mathrm{~ms}$, and PER 0.000001 . It may be assigned to services such as mission critical delay sensitive signaling;

- New value \#5: default priority level 6, PDB $400 \mathrm{~ms}$, and PER 0.000001 . It may be assigned to services such as mission critical data.

Among the above new PQI values, new values \#1, \#2, and \#3 are defined for GBR resource type, and the default averaging window is $2000 \mathrm{~ms}$. New values \#4 and \#5 are defined for non-GBR resource type.

Although the support for these public safety specific PQI values is still under discussion, it is hopeful that the QoS requirements associated with these new PQIs can be met by adapting existing NR SL QoS handling. To be more specific, new values \#1, \#2, and \#3 may be met by PQI = 91 in Table 14 directly. It is also very likely that new value \#4 may be met by adapting $\mathrm{PQI}=91$ properly, because it may be feasible to improve the packet error rate by one order of magnitude when the packet delay budget is relaxed by 40 times and the priority level is promoted. There is some uncertainty about meeting the QoS requirements of new value \#5 considering the downgraded priority level and the stringent packet error rate. However, combining the $\mathrm{QoS}$ handling of $\mathrm{PQI}=56$ and $\mathrm{PQI}=91$ may be a promising starting point. 
Please note that these new PQI values are still under discussion, and have not been standardized by 3 GPP at the time this document was drafted. Please check the latest version of [26] for the up-to-date, standardized PQI values.

\subsection{Summary}

As can be seen from above sections, the QoS support for direct mode communication over SL has been greatly enhanced in NR. In contrast to LTE's limited and simplified QoS control for ProSe communications, NR's QoS mechanism for SL communication is fullfledged, and is a miniature version of that for communication over the Uu interface. With the enhanced QoS framework enabled for NR SL communication and the ongoing standardization efforts to support public safety applications, we expect that $5 \mathrm{G}$ NR has great potential to meet QoS requirements of direct mode communications for public safety services.

\section{LTE/NR Coexistence}

\subsection{Introduction}

Operators have already rolled out 5G NR in select markets. However, the majority of users are still equipped with $4 \mathrm{G}$ LTE phones and it will take some time before a full nationwide $5 \mathrm{G}$ network becomes available. In addition, most frequency bands below $2.5 \mathrm{GHz}$ are FDD and are already occupied, such as Band n14. Refarming, which would require to completely stop using LTE and switch to NR, would be expensive and cause service disruption for many users. To address this spectrum limitation and enable a smooth transition, 3GPP has defined mechanisms to support Dynamic Spectrum Sharing (DSS) [29]. In the following sections, we first describe DSS with an emphasis on the UL then discuss if it can be leveraged to support SL communications.

\subsection{DSS Overview}

DSS provides mechanisms for sharing part of the spectrum between LTE and NR to enable a smooth transition between the two technologies. This is accomplished by enabling coordination between eNodeBs and gNodeBs to ensure that specific subframes and RBs are used by one technology at a time. DSS supports both time multiplexing (for static and semi-static coordination) and frequency multiplexing (for more dynamic coordination). NR numerology 0 and 1 can be used for DSS, but each brings its own set of constraints with regards to how it can be used, such as granularity of time and frequency that can be shared. Coordination between neighboring cells is done by exchanging two types of information over the Xn interface: configuration and data allocation.

Configuration information is exchanged via either the XN SETUP procedure or the NG-RAN NODE CONFIGURATION UPDATE, as shown in Fig. 18. Those procedures are used to provide or update application-level configuration data needed for two NG-RAN 
nodes to interoperate properly over the $\mathrm{Xn}-\mathrm{C}$ interface, which is the signaling plane between NG-RAN nodes. An NG-RAN node is defined as either an ng-eNodeB or a gNodeB.

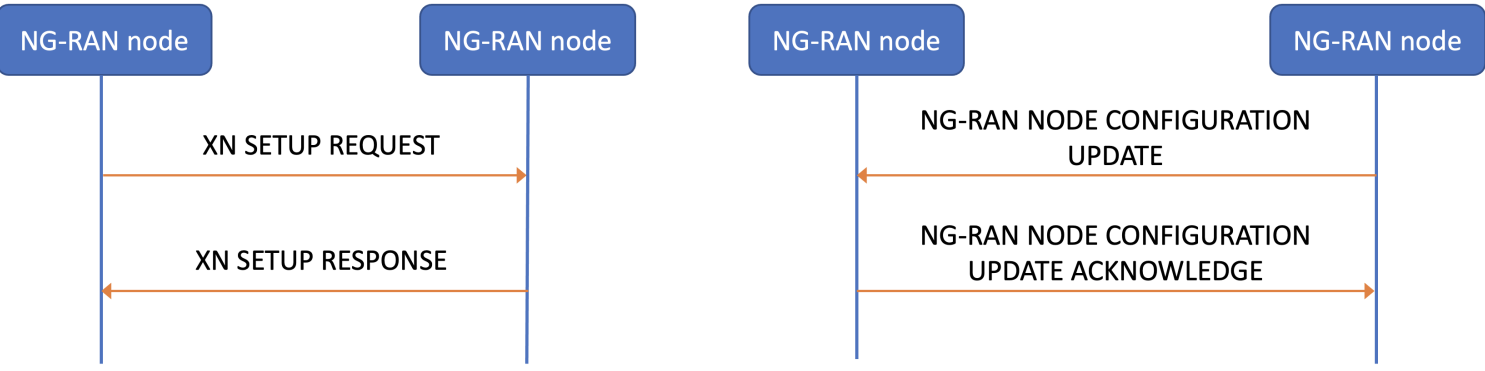

Fig. 18. Xn setup and Node Configuration messages [29].

When an eNodeB shares its configuration, a neighboring node can compute the list of resources that will be used to carry cell specific recurring signals, such as DL and UL control and synchronization signals. A portion of the message is dedicated to providing Served Cell Information E-UTRA and contains the following information:

- Physical Cell ID (PCI), PLMNs IDs.

- Band information (frequency and bandwidth).

- NarrowBand-Internet of Things (NB-IoT) information.

- TDD allocation

- Antenna port, Physical Random Access Channel (PRACH) configuration, MulticastBroadcast Single-Frequency Network (MBSFN) configuration.

- Protected E-UTRA resource indication (i.e., DL and UL control/reference signals protected and not subject to (data) resource coordination).

- Activation System Frame Number (SFN).

- Physical Downlink Control Channel (PDCCH) and MBSFN control region lengths.

- A list of up to 16 patterns, each containing:

* Type: E-UTRA DL non-CRS, E-UTRA DL CRS, or E-UTRA UL.

* Bitmap of REs occupied by the protected signal within one PRB (if bitmap contains all ones then it is PRB-level granularity). For example, in LTE UL, the length of the bitmap would be 84 because there are 12 subcarriers and 7 symbols per PRB.

* Bitmap with frequency pattern where protected pattern applies inside the carrier bandwidth. The bitmap size equals the number of PRBs in carrier bandwidth, e.g., 50 for a bandwidth of $10 \mathrm{MHz}$. 
* Periodicity of the pattern (e.g., 1 = every slot, 2 = every other slot, etc.) and start of pattern (in subframe).

The SFN is necessary to indicate when the configuration will be valid in case it changes. We also notice that the MBSFN configuration is included. This is an important piece to enable sharing between LTE and NR because it allows the use of subframes configured for MBSFN that have a lower overhead compared to normal DL subframes, since there are no reference signals broadcasted in the data region. More information can be found in [30], where there is a detailed analysis of DSS in the DL, including its impact on the performance. With regards to UL, we observe that all the necessary control and synchronization signals are supported. In particular, there is the PRACH configuration to determine when and where the PRACH channel occurs. The Protected E-UTRA resource indication can be used to specify regions allocated for Physical Uplink Control Channel (PUCCH) and SRS transmissions. An example is shown in Fig. 19, where a PUCCH Type 2 region and an SRS region are defined for frames using normal $\mathrm{CP}$ on a $10 \mathrm{MHz}$ band. For the $\mathrm{PUCCH}$ region, all the REs in the slot are used, thus the 84 bits (e.g. 12 subcarriers $x 7$ symbols) are set to 1. The frequency pattern has the first and last three bits set to 1 to indicate that it affects the first and last three RBs of the slot. The time periodicity is 1 because the PUCCH repeats every slot, and the start time is 1 to indicate slot 0 of subframe 0 of the SFN specified in the message. For the SRS region, which occupies only the last symbol of the second slot, only the last 12 bits of the resource footprint are set to 1 . The frequency pattern excludes the first and last three RBs since those are already occupied by the PUCCH. Finally, the time periodicity is set to 2, because the SRS occurs every other slot, and the start time is set to 2 to indicate that it starts on slot 1 of subframe 0 of the SFN specified in the message.

$\begin{array}{ll} & \text { Resource type: E-UTRA UL } \\ \text { Intra-PRB Protected Resource Footprint: } \\ \text { 11111111111111111....111111111 ( } 84 \text { bits) } \\ \text { PUCCH } \quad \text { Protected Footprint Frequency Pattern: } \\ \text { 111000000...000000111 (50 bits) } \\ \text { Protected Footprint Time-periodicity: } 1 \\ \text { Protected Footprint Start Time: } 1 \\ \\ \text { Resource type: E-UTRA UL } \\ \text { Intra-PRB Protected Resource Footprint: } \\ \text { 000....0000000000011111111111 ( } 84 \text { bits) } \\ \text { Protected Footprint Frequency Pattern: } \\ \text { 0001111111...1111000 (50 bits) } \\ \text { Protected Footprint Time-periodicity: } 2 \\ \text { Protected Footprint Start Time: } 2\end{array}$

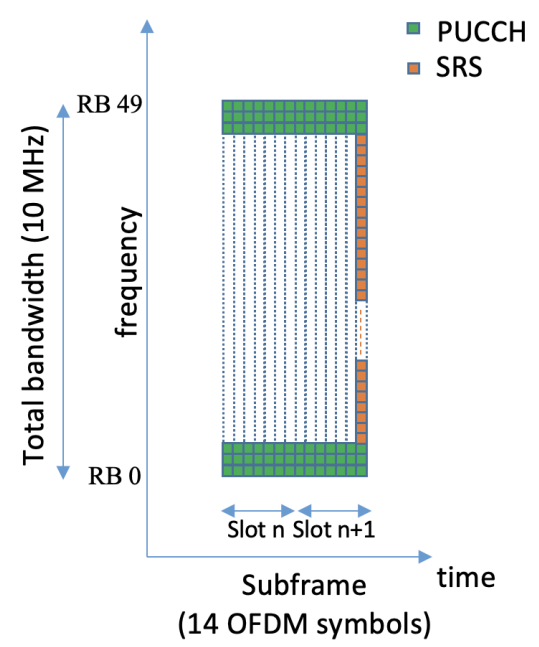

Fig. 19. Example of protected resources pattern definitions in UL.

After defining areas that are dedicated to E-UTRA control and synchronization signals, neighboring cells can coordinate the remaining resources used for data transfer. This is 
achieved by exchanging E-UTRA-NR CELL RESOURCE COORDINATION messages, as shown in Fig. 20.
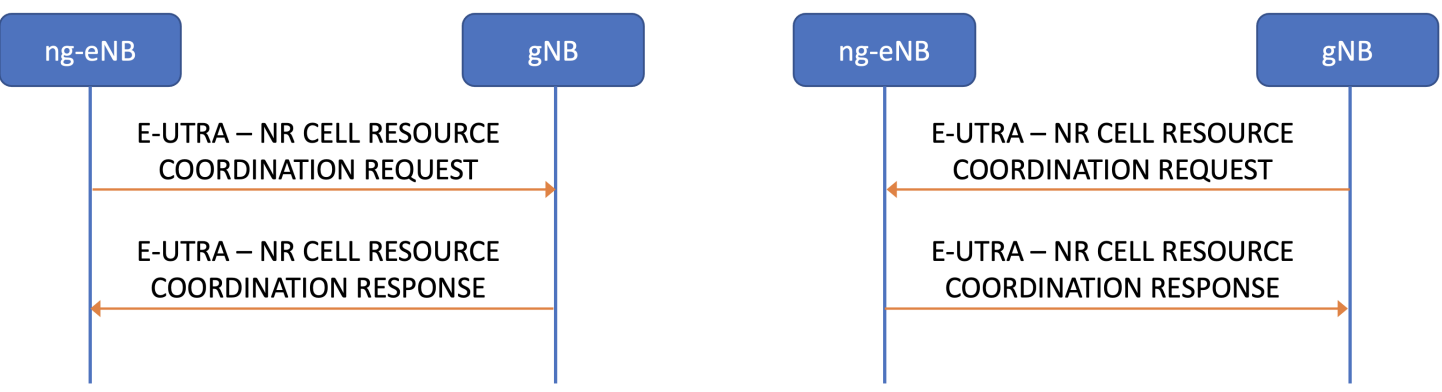

Fig. 20. Xn resource coordination messages [29].

Both eNodeBs and gNodeBs can initiate the request, each providing the list of resources it plans to use via the Data Traffic Resource Indication field containing the following information:

- SFN of the receiver node in which the agreement is valid.

- Whether the node is providing resource indication for the UL only or for both the DL and the UL.

- Bitmap(s) with resources expected to be used for E-UTRA Physical Downlink Shared Channel (PDSCH) and/or Physical Uplink Shared Channel (PUSCH).

- Size 6 to 17600 , with each bit representing 1 PRB starting with lowest subcarrier frequency in the carrier starting with subframe 0 (Minimum size of $6=$ number of RBs in 1 subframe with $1.4 \mathrm{MHz}$ band).

- Bitmap must be multiple of UL/DL bandwidth, i.e., must be multiple of consecutive subframes. Therefore with $10 \mathrm{MHz}$ band (50 RBs), the maximum number of subframes that can be specified is 352. An example of such bitmap and its visual representation is shown in Fig 21.

- List of reserved subframes where the resource allocation does not hold

Due to the limited number of subframes that can be specified and the dynamic nature of the traffic, coordination messages have to be exchanged periodically between neighboring nodes. How often will depend on the operator and the ability to predict future allocations. Similarly, the algorithms used to converge to an agreed spectrum sharing arrangement, in case two or more cells decide to use the same resources, will be operator specific. 

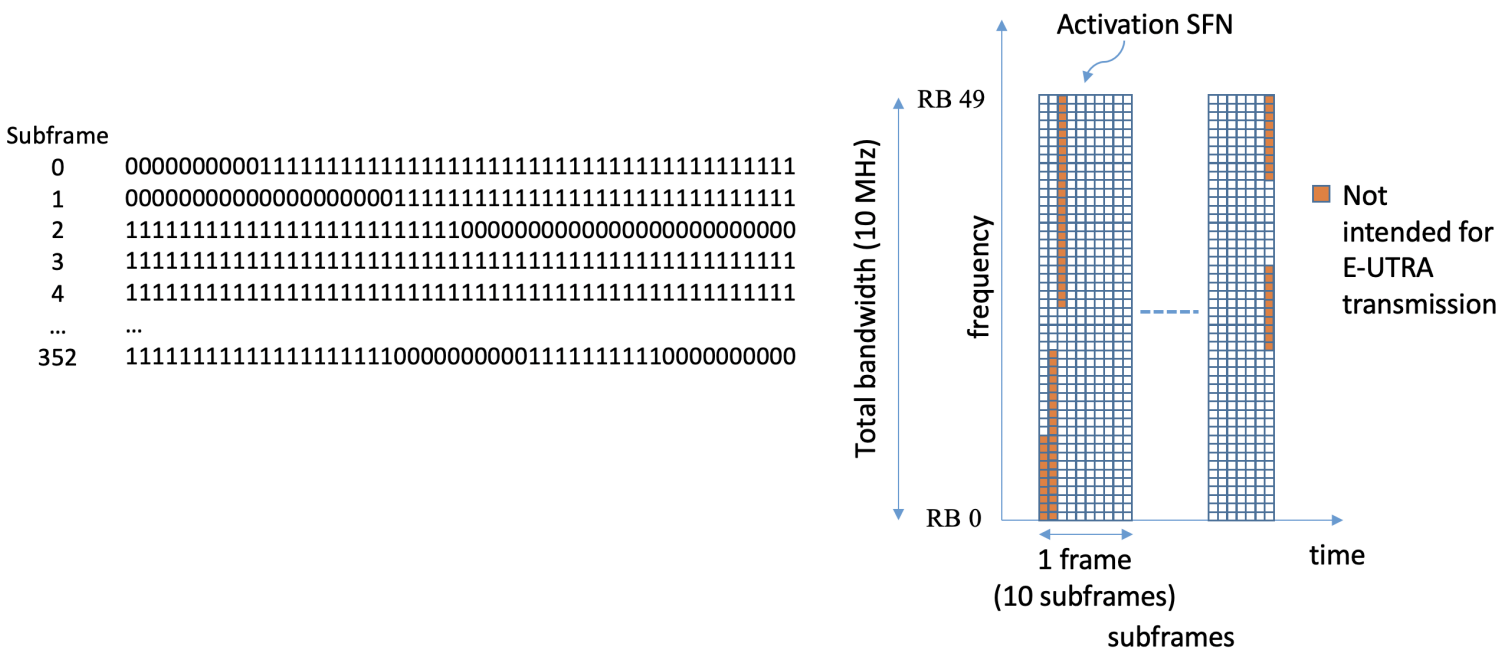

Fig. 21. Example use of the Data Traffic Sharing Resource Indication field.

\subsection{Sidelink Considerations}

As currently defined, DSS provides both DL and UL resource coordination by specifying broadcast, control, and shared resource information. In order to support deployments with LTE SL and/or NR SL, additional resource information must be communicated. Table 15 provides a summary of the various channels used by each technology, the frequency of transmissions, and RB locations.

Table 15. LTE SL and NR SL channels

\begin{tabular}{|c|c|c|}
\hline Channel & LTE & NR \\
\hline $\mathrm{PSBCH}$ & $\begin{array}{l}\text { When activated by transmitting UE, ev- } \\
\text { ery } 40 \mathrm{~ms} \text { on the center six RBs of the } \\
\text { frequency band. }\end{array}$ & $\begin{array}{l}\text { Periodicity of } 16 \text { frames, with up to } 64 \\
\text { transmissions within period based on } \\
\text { numerology (one transmission for nu- } \\
\text { merology } 0 \text { ). }\end{array}$ \\
\hline PSCCH & $\begin{array}{l}\text { Periodic allocation between } 40 \mathrm{~ms} \text { and } \\
320 \mathrm{~ms} \text {, up to } 40 \mathrm{~ms} \text { long. }\end{array}$ & Transmitted in same slot as PSSCH. \\
\hline PSSCH & $\begin{array}{l}\text { Periodic allocation between } 40 \mathrm{~ms} \text { and } \\
320 \mathrm{~ms} \text {. Number of subframes and RBs } \\
\text { based on pool configuration. }\end{array}$ & $\begin{array}{l}\text { Inside Sidelink BWP. Number of sub- } \\
\text { frames and RBs based on pool config- } \\
\text { uration. }\end{array}$ \\
\hline \multicolumn{3}{|l|}{ Physical } \\
\hline $\begin{array}{l}\text { Sidelink } \\
\text { Discovery }\end{array}$ & $\begin{array}{l}\text { Periodic allocation between } 320 \mathrm{~ms} \\
\text { and } 10.24 \mathrm{~s} \text {. Number of subframes and }\end{array}$ & $\begin{array}{l}\text { N/A. Not defined in Rel-16. Discovery } \\
\text { messages may be sent using PSSCH in }\end{array}$ \\
\hline $\begin{array}{l}\text { Channel } \\
\text { (PSDCH) }\end{array}$ & RBs based on pool configuration. & Rel-17. \\
\hline PSFCH & N/A & Transmitted in same slot as PSSCH. \\
\hline
\end{tabular}

There are different ways to address the current limitations depending on the technology 
and allocation type:

- For out-of-coverage operation, which would not require DSS, it is possible to preconfigure non overlapping resource pools for both LTE and NR SL. Those could be separated in frequency, time, or both.

- For in-coverage scenarios and UE selected resource pools, it would be beneficial for the eNodeB or gNodeB to send the resource pool configuration as part of the SETUP or NG-RAN NODE CONFIGURATION UPDATE in order to limit overhead. This is because the eNodeB or $\mathrm{gNodeB}$ is not aware of the specific resource selection made by the UEs, and therefore it would be preferable that a neighboring node does not use any of the resources allocated for SL. This however, would require modifications to the 3GPP specifications to add SL specific information elements.

- For in-coverage scenarios and scheduled mode, where the eNodeB/gNodeB selects specific resources for the SL transmissions, it could be possible to use either the SETUP or NG-RAN NODE CONFIGURATION UPDATE messages, or the CELL RESOURCE COORDINATION message. In the former case, overhead is reduced but may lead to lower efficiency if there is not a lot of SL traffic. This is because the eNodeB/gNodeB would try to completely avoid all resources allocated for SL, as in the case for UE selected resource pools. With the latter, an eNodeB or gNodeB could indicate specific resources allocated to SL and provide finer control over the shared spectrum. If there is no SL traffic, the eNodeB/gNodeB could transmit in the UL even in resources that are part of the SL resource pools. Either option would also require modifications to the $3 \mathrm{GPP}$ specifications.

\subsection{Summary}

In this section, we described DSS specified by 3GPP to facilitate coexistence between LTE and NR and allow efficient use of the spectrum, which is very limited in the lower bands. Based on the current specifications in Release 16, we noted several limitations with regards to supporting coexistence when considering both LTE and NR SL communications.

\section{Ongoing Standardization}

\subsection{Introduction}

3 GPP is currently working on Rel-17 specifications, and a firm timeline for the release has been approved at the December 2020 plenary meeting: the Stage 2 functional freeze is planned for June 2021, the Stage 3 protocol freeze for March 2022 and finally, the protocol coding freeze is aimed for June 2022 [31]. Rel-17 will implement several enhancements and new features to further support direct mode communications in the 5G NR architecture. In this section, we provide an overview of the related 3GPP study and work items that were prioritized for inclusion in Rel-17. 


\subsection{Proximity based Services in 5GS work items}

Proximity based Services, or ProSe, was the first technology to support direct communication between UEs for LTE systems. LTE ProSe was introduced in Rel-12 and led to the creation of the LTE SL, which was later enhanced to support LTE V2X. ProSe use cases for public safety communications identified in [32] comprise in-network and off-network operation of device discovery, one-to-one, group, and broadcast traffic exchange between first responder's UEs in proximity, and coverage extension with UE-based relays. More recently, commercial ProSe use cases were identified in [33], such as interactive gaming and augmented reality between UEs in proximity, real-time data exchange between nearby sensors, and lightweight wearable devices connected to computationally capable UEs. Given that these ProSe use cases are associated to different requirements than those of V2X, and that ProSe functionalities are key factors to the support of mission critical communications in 5G System (5GS) within the NR architecture [34], it was important to study their feasibility within Rel-16 NR SL and V2X solutions, and to identify any extension needed to support them in Rel-17.

\subsubsection{Study Item phase}

The study item entitled Study on System enhancement for Proximity based Services in 5GS (FS_5G_ProSe) [35] and its resulting technical report TR 23.752 [36] focused on identifying technological gaps and potential solutions to support ProSe on the 5G NR architecture. The FS_5G_ProSe study evaluated direct discovery, direct communication, and UE-based relay ProSe functionalities, and aimed to find common solutions to satisfy commercial and public safety requirements. In addition, the Study on NR Sidelink Relay (FS_NR_SL_Relay) complemented the FS_5G_ProSe study by focusing on radio access network aspects of the UE-based relay ProSe functionalities.

NR ProSe Direct Discovery The definition of direct discovery did not change from LTE ProSe to NR ProSe. Based on [36], the ProSe direct discovery procedure is still used for a UE to discover or be discovered by other $\mathrm{UE}(\mathrm{s})$ in proximity over the PC5 interface (as it was the case for LTE ProSe). The UE can discover other UE(s) with interested application(s) and/or interested group(s) using the ProSe direct discovery procedure. This feature aims to achieve the common direct discovery procedure for discovering a 5G ProSe enabled UE, 5G ProSe U2N Relay, or a 5G ProSe UE-to-UE (U2U) Relay.

Multiple aspects (architecture, message, resource pool, protocol stack) of the direct discovery are part of the ongoing work by the standardization. However, certain suggestions have more approval than others including:

- Direct discovery message would be taken as a new signaling in the ProSe layer, separate from PC5-S signaling. The ProSe layer would indicate to the AS layer whether the signaling is a discovery message or PC5-S signaling. 
It is feasible to configure a dedicated priority value for the discovery message, which is different from the other SL Signaling Radio Bearer (SRB).

- Resource pool to transmit discovery messages can be either shared with or separated from the data transmission resource pool.

In case of a shared resource pool, a new Logical Channel ID (LCID) is introduced for discovery messages, i.e., discovery messages are carried by a new SL SRB.

For a separated resource pool, discovery messages are treated equally with each other during the Logical Channel Prioritization (LCP) procedure. It does not seem necessary to introduce a new LCID for discovery messages since the discovery message can be identified by a different resource pool.

It is highly expected that new SRB and LCID are introduced for discovery messages since discovery messages are not a part of PC5-S signaling and they are neither PC5 RRC messages nor SL data.

- Since the direct discovery message is taken as new signaling in ProSe layer separately from PC5-S signaling, the protocol stack of direct discovery can be depicted as Fig. 22, in which the Discovery is located on top of the PDCP, Radio Link Control (RLC) and MAC sublayers.

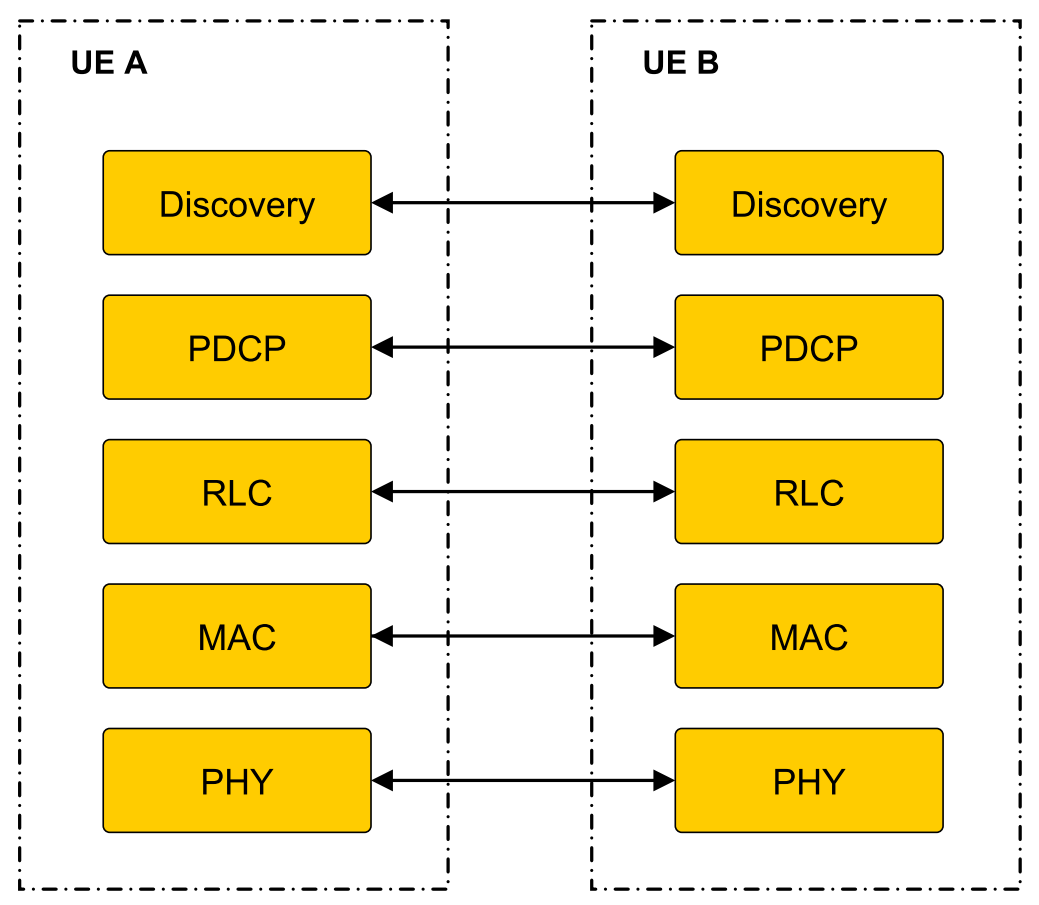

Fig. 22. Protocol stack for direct discovery.

NR ProSe Direct Communication Direct communication is the functionality that defines one-to-one and one-to-many direct traffic exchange between nearby UEs for ProSe. 
ProSe direct communication should allow first responders in proximity to use mission critical applications to communicate even in cases of network unavailability. On the commercial side, the use of direct communication between UEs in proximity, instead of the network, is expected to provide enhanced experience for interactive applications such as gaming or augmented reality. Moreover, ProSe direct communication is the base for the UE-based relaying functionalities and coverage extension use cases that will be presented in the next section.

Similar to V2X applications over PC5, UEs using ProSe direct communication will use the SL over the PC5 interface to transmit and receive data and signaling messages, if applicable. Thus, this functionality was found feasible for 5G NR systems in [36] by reusing the V2X communication over PC5 reference point mechanisms defined for Rel-16 in [26], which comprise unicast, broadcast, and groupcast direct communication between UEs using the NR SL. These mechanisms, together with several extensions such as the support of Internet Protocol version 4 (IPv4), Address Resolution Protocol (ARP), Ethernet and Unstructured data unit types, and the support of enhanced QoS handling, are envisioned in [36] to fully support ProSe direct communication use cases.

NR Sidelink Relays Two UE-based relay capabilities were studied in [36]: UE-to-Network (U2N) relay, where a UE extends the network connectivity to another nearby UE by using direct communication; and UE-to-UE (U2U) relay, where a UE uses two direct communication links to connect two UEs in its proximity that otherwise are not able to communicate.

The U2U relay functionality was not part of the LTE ProSe specification, and its inclusion on NR ProSe can be beneficial for public safety communications range extension for both in-network and off-network use cases. U2N relay functionality is fundamental for network coverage extension for public safety interventions in remote areas, as well as, for wearable devices tethering in commercial use cases (e.g., sensors, virtual reality headsets).

The Study on NR Sidelink Relay ( FS_NR_SL_Relay) and resulting technical report TR 38.836 [37] complemented the FS_5G_ProSe study focusing on radio access network aspects of the UE-based relay ProSe functionalities. Both, the U2N and U2U relay functionalities were found feasible by reusing some of the LTE ProSe and NR V2X components, and defining new architectures and protocols.

LTE U2N relay functionality uses a Layer 3 (L3) architecture in which the relay of data packets in the PC5 interface is performed at the network layer, and UEs connected to a L3 U2N relay are transparent to the network. For NR UE-based relay solutions, two architectures were found feasible in [36] and [37]: the L3 architecture as in LTE, and a newly defined Layer 2 (L2) architecture in which the relaying in the PC5 interface occurs within the L2, over the RLC sublayer. A UE connected to an L2 U2N relay is expected to be seen by the network as a regular UE (i.e., as if it was directly connected to the network), which gives the network control of the connection and services, but requires the definition of several new mechanisms not present or needed in the L3 architecture. These would include, at minimum, a PC5 to Uu adaptation layer for RLC channels and bearer mapping, indirect paging and system information forwarding, and network controlled path switching. 
Architecture-independent components from previous releases were agreed to be used as baselines for Rel-17. The relay discovery models and the relay selection criteria and procedures will be based on the LTE ProSe solutions. Rel-16 V2X unicast communication protocol will be used as a baseline for both architectures. This includes the establishment, maintenance, modification, and release of a connection between a UE and UE-based relay, and the corresponding data exchange using direct communication. Finally, QoS aspects of Rel-16 will be reused and extended to support mapping between PC5 and Uu QoS parameter and rules to ensure end-to-end QoS for a UE connected to a relay UE.

\subsubsection{Work Item phase}

As shown in the previous section, the technical working groups involved in FS_5G_ProSe and FS_NR_SL_Relay studies found the ProSe functionalities feasible to be implemented within the 5G NR architecture in Rel-17. Currently, the corresponding technical working groups are developing the normative specification of the identified solutions under two work items:

- 5G_ProSe work item, with the current scope described in [38], and

- NR_SL_Relay work item, with the current scope described in [39].

5G_ProSe technical specifications are being documented in TS 23.304 [40], and technical solutions are expected to impact other specification documents as listed in [38] and [39]. At the time of writing this document, ProSe discovery and ProSe communication normative work is ongoing, while both L2 and L3 architectures for U2N relay functionality were recently approved and the normative work should start shortly. Due to time and workload constraints, the U2U relay functionality was excluded from the current Rel-17 work items scopes.

\subsection{NR sidelink enhancement work item}

The NR sidelink enhancement (NR_SL_enh) work item [41] is ongoing at the time of writing this document and it aims to specify radio solutions for Rel-17 that can enhance the NR SL for all services expected to use it, including V2X, public safety, and other commercial use cases.

3GPP radio access network working groups identified important NR SL topics that were not fully covered by the solutions specified in Rel-16, and that could be addressed in Rel-17 [42]. This includes power savings, enhanced reliability, reduced latency, increased data rate, support of new carrier frequencies and operation scenarios, increased spectral efficiency, and enhanced coverage. The topics related to UE power saving and to enhanced reliability and reduced latency were selected to be the main focus of the NR_SL_enh work item specifications for Rel-17, although objectives related to the support of new carrier frequencies and restricted use of frequency ranges are also present in the work item. It was

also determined that extensions to the NR SL evaluation methodology should be discussed 
to include public safety and commercial use cases in addition to existing V2X evaluation scenarios. The current scope of the NR_SL_enh work item can be found in [41]. The topics related to coverage enhancements are to be covered by work items specifying UE-based relay technologies (i.e., $F S \_N R \_S L \_$Relay and $5 G \_$ProSe), and the specification of the other topics in future releases is still an open subject at the time of writing.

Some of the NR SL use cases expected to be supported in Rel-17 have strict reliability and latency requirements. The RAN working groups found that the solutions specified in Rel-16 may not satisfy those requirements in all conditions, specially when using resource allocation mode 2, i.e., when each UE autonomously selects its resources, and during busy channel conditions [42]. Thus, the NR_SL_enh work item currently focuses on studying and specifying solutions that can further improve Rel-16 reliability and reduce latency within resource allocation mode 2 . In particular, inter-UE resource allocation coordination schemes are being considered, in which UEs exchange information that can assist the resource selection of the others. This can further reduce resource collision and thereby improve reliability.

The power savings enhancements are motivated by Rel-17 uses cases for the NR SL, which go beyond the vehicular communication defined on Rel-16, in which devices do not have strong power limitations. In Rel-17, battery-constrained portable devices such as smartphones, head or backpack mounted devices, and wearables, are expected to use the NR SL in order to support advanced V2X use cases, such as Vulnerable Road User (VRU) warning, and also to enable ProSe use cases, such as direct communication for public safety mission critical operations and commercial interactive applications. Two main aspects were identified in the work item to enhance UE power savings: reducing sensing active time used for resource allocation, e.g., by using partial sensing or no sensing at all; and introducing SL Discontinuous Reception (DRX) in which the devices are active for data and control reception only when deemed necessary. The random resource selection with no sensing and the partial sensing schemes of LTE SL, introduced in Rel-14, are going to be used as a baseline for the power efficient resource allocation schemes of Rel-17. How to adapt them to the NR SL and whether another solution may be needed are under discussion at the time of writing. The NR SL DRX will reuse the Uu interface design aspects and it is expected to be supported in all direct communication cast types, i.e., broadcast, groupcast, and unicast, and for all coverage scenarios. Active and inactive patterns, durations and mechanisms, together with active time alignment for UE communication with each other, and with the $\mathrm{Uu}$ in the in-network case, will be studied and specified within the NR_SL_enh work item.

\subsection{Enhanced V2X services work items}

Two study items related to enhancing V2X services are present in the Rel-17 timeline:

- Study on architecture enhancements for 3GPP support of advanced V2X services Phase 2 (FS_eV2XARC_Ph2) [43] with findings being reported on TR 23.776 [44]

- Study on enhancements to application layer support for V2X services (FS_eV2XAPP) [45] with findings being reported in TR 23.764 [46]. 
The FS_eV2XARC Ph2 study item aims to identify and analyze solutions that enhance Rel-16 V2X specifications to support V2X services for pedestrian UEs in Rel-17, which may have power and computation limitations with respect to the vehicular UEs supported in Rel-16. In particular, the study examines how SL DRX, as studied in NR_SL_enh work item for power saving (described in Section 8.3), can affect the V2X services that will run over the NR PC5 and used by pedestrian UEs. To judge the feasibility of SL DRX for V2X applications, the study will evaluate solutions to determine by what means DRX should be configured, how to maintain QoS requirements while using it, and how to coordinate simultaneous $\mathrm{V} 2 \mathrm{X}$ services for maximizing power saving.

The FS_eV2XAPP study item aims to analyze Rel-16 specifications related to the support of V2X in 5GS, and to identify the impact they have on the V2X application layer. The focus is on identifying key issues and proposing solution recommendations to enable the application layer support for V2X services in 5GS for both NR Uu and NR PC5 interfaces usage. At the time of writing, 16 key issues have been identified in [44], and many of them have implications related to the use of PC5 and direct communication for V2X services: i) application layer support for PC5 QoS parameters provisioning, monitoring, and adaptation; ii) NR PC5 session handling for direct communication in support of tele-operated driving; iii) NR PC5 provisioning in multi-operator V2X scenarios; iv) usage, selection, provisioning, and configuration of the different cast modes (unicast, broadcast or groupcast) by the application layer; and v) switching modes between NR Uu and NR PC5 for $\mathrm{V} 2 \mathrm{~V}$ communications. The corresponding solutions to be used as baselines for normative work are described in [44] as well, and the $e V 2 X A P P$ work item is currently ongoing to specify the features identified in the $F S_{-} e V 2 X A P P$ study item. The current scope can be found in [47].

\subsection{Summary}

The NR SL was defined in Rel-16 to support direct mode communication for several V2X services. In Rel-17, NR SL specifications are meant to expand to support additional V2X services (e.g., VRU) and ProSe features, including public safety direct communication. In this section, we described Rel-17 study and work items related to the enhancement of the NR SL to support those services (NR_SL_enh), the definition of NR ProSe and its evolution from LTE ProSe (FS_5G_ProSe, 5G_ProSe, FS_NR_SL_Relay, and NR_SL_Relay) and the impact and enhancements needed in the V2X architecture to support the new services $\left(F S \_\right.$eV2XARC_Ph2) and to work within 5G NR systems (FS_eV2XAPP and $\left.e V 2 X A P P\right)$. 


\section{Conclusion}

In this study, we evaluated the 3GPP specifications enabling D2D communication in 5G NR in order to understand the improvements and limitations to fully support direct mode communication for first responders.

We started by looking at how resources are being allocated and services provisioned for V2X in Rel-16. We focused on configurations related to the PC5 interface for SL communication. Since V2X services can operate both on- and off-network, it provides a good foundation for how ProSe services could be managed. Through the analysis of the specifications, we highlighted possible issues that will need to be addressed especially regarding the co-existence of $\mathrm{V} 2 \mathrm{X}$ and non-V2X applications and how to deal with possible conflicting resource allocations. In the Physical Layer section, we further described the mechanisms used to transmit and receive packets over the SL channels. We highlighted the flexible numerologies, detailed the various SL channels, and described new features such as the feedback channel, enhanced coding, and MIMO, which are expected to increase performance compared to LTE. The increase of capacity is discussed in the capacity section where we conducted an initial analysis of NR SL for the unicast case. We derived formulas for the various overheads and studied the impact of numerology, modulation, and blind retransmissions. The results showed that there is a potential capacity increase for NR SL compared to LTE SL with the actual gain dependent on the system configuration. In the Quality of Service section, we described the mechanisms defined for the Uu interface as well as the PC5 interface in NR. The analysis of the specifications show that there are significant enhancements compared to LTE to handle QoS, including special PQI values for public safety traffic. We also highlighted the MCR capability that defines the geographical area where QoS requirements apply for groupcast communication, a feature that can be beneficial for first responder communications using MCPTT group calls. In the LTE/NR Coexistence section, we described DSS, which allows the sharing of resources between LTE and NR, and showed that the current specifications need to be improved in order to take into account SL communication. Finally, we addressed the continuous discussions by the 3GPP groups regarding discovery (architecture, protocol stack, message content, resource pool), relays (types, layers, architecture, selection criteria) and other NR enhancements (e.g., power consumption and battery limitations, NR Uu and NR PC5 interoperability). It is expected to have those features added to Rel-17 after further review and approval.

The findings of this study confirm that NR SL can provide improved performance for D2D communication which can benefit direct mode communication for first responders. However, as discussed throughout this document, there are several limitations in the current specifications that need to be addressed to support non-V2X applications. In addition, there is a need to provide measurement tools to quantify the performance improvement and determine the best set of parameters for the various situations facing first responders. We plan to continue working with FirstNet and public safety agencies to prioritize further research based on the findings shown in this document and recognize that other topics, such as privacy, security, and range must also be considered. 


\section{Acknowledgments}

The authors would like to thank Erickson Trejo-Reyes at FirstNet for providing additional insights and perspectives related to challenges facing first responders and network operations.

\section{References}

[1] 3GPP (2020) NR; User Equipment (UE) radio transmission and reception; Part 2: Range 2 Standalone (3rd Generation Partnership Project (3GPP)), Technical Specification (TS) 38.101-2. Version 16.5.0 Available at https://portal.3gpp.org/ desktopmodules/Specifications/SpecificationDetails.aspx? specificationId=3284.

[2] Lien S, Deng D, Lin C, Tsai H, Chen T, Guo C, Cheng S (2020) 3GPP NR sidelink transmissions toward 5G V2X. IEEE Access 8:35368-35382. https://doi.org/10.1109/ ACCESS.2020.2973706

[3] 3GPP (2020) Vehicle-to-Everything (V2X) services in 5G System (5GS); Stage 3 (Release 16) (3rd Generation Partnership Project (3GPP)), Technical Specification (TS) 24.587. Version 16.3.0 Available at https://portal.3gpp.org/desktopmodules/ Specifications/SpecificationDetails.aspx?specificationId $=3640$.

[4] 3GPP (2020) Vehicle-to-Everything (V2X) services in 5G System (5GS); User Equipment (UE) policies; Stage 3 (Release 16) (3rd Generation Partnership Project (3GPP)), Technical Specification (TS) 24.588. Version 16.4.0 Available at https://portal.3gpp.org/desktopmodules/Specifications/SpecificationDetails. aspx ?specificationId=3641.

[5] ISO (2021) Intelligent transport systems - cooperative systems - globally unique identification. Available at https://standards.iso.org/iso/ts/17419/TS17419\% 20Assigned\%20Numbers/TS17419_ITS-AID_AssignedNumbers.pdf.

[6] 3GPP (2020) NR; User Equipment (UE) radio transmission and reception; Part 1: Range 1 Standalone (Release 16) (3rd Generation Partnership Project (3GPP)), Technical Specification (TS) 38.101-1. Version 16.5.0 Available at https://portal.3gpp.org/ desktopmodules/Specifications/SpecificationDetails.aspx? specificationId=3283.

[7] 3GPP (2020) NR; Radio Resource Control (RRC) protocol specification (Release 16) (3rd Generation Partnership Project (3GPP)), Technical Specification (TS) 38.331. Version 16.2.0 Available at https://portal.3gpp.org/desktopmodules/Specifications/ SpecificationDetails.aspx? specificationId $=3197$.

[8] 3GPP (2020) Overall description of Radio Access Network (RAN) aspects for Vehicle-to-everything (V2X) based on LTE and NR (Release 16) (3rd Generation Partnership Project (3GPP)), Technical Report (TR) 37.985. Version 16.0.0 Available at https://portal.3gpp.org/desktopmodules/Specifications/SpecificationDetails. aspx? specificationId=3601.

[9] 3GPP (2020) NR; Physical channels and modulation (Release 16) (3rd Generation Partnership Project (3GPP)), Technical Specification (TS) 38.211. Ver- 
sion 16.3.0 Available at https://portal.3gpp.org/desktopmodules/Specifications/ SpecificationDetails.aspx? specificationId $=3213$.

[10] 3GPP (2020) NR; Physical layer procedures for control (Release 16) (3rd Generation Partnership Project (3GPP)), Technical Specification (TS) 38.213. Version 16.3.0 Available at https://portal.3gpp.org/desktopmodules/Specifications/ SpecificationDetails.aspx?specificationId $=3215$.

[11] 3GPP (2020) NR; Multiplexing and channel coding (Release 16) (3rd Generation Partnership Project (3GPP)), Technical Specification (TS) 38.212. Version 16.3.0 Available at https://portal.3gpp.org/desktopmodules/Specifications/ SpecificationDetails.aspx? specificationId $=3214$.

[12] 3GPP (2020) NR; Physical layer procedures for data (Release 16) (3rd Generation Partnership Project (3GPP)), Technical Specification (TS) 38.214. Version 16.3.0 Available at https://portal.3gpp.org/desktopmodules/Specifications/ SpecificationDetails.aspx?specificationId=3216.

[13] 3GPP (2018) Revised WID: Enhancements on MIMO for NR (3rd Generation Partnership Project (3GPP)), Work Item Description RP-182863. Available at https://portal.3gpp.org/ngppapp/CreateTDoc.aspx?mode=view\&contributionUid= RP- 182863.

[14] Vook FW, Hillery WJ, Visotsky E, Tan J, Shao X, Enescu M (2018) System level performance characteristics of sub-6ghz massive mimo deployments with the 3gpp new radio. 2018 IEEE 88th Vehicular Technology Conference (VTC-Fall), , pp 1-5. https://doi.org/10.1109/VTCFall.2018.8690560

[15] 3GPP (2019) Study on NR Vehicle-to-Everything (V2X) (3rd Generation Partnership Project (3GPP)), Technical Report (TR) 38.885. Version 16.0.0 Available at https://portal.3gpp.org/desktopmodules/Specifications/SpecificationDetails. aspx?specificationId $=2934$.

[16] 3GPP (2017) Study on new radio access technology physical layer aspects (3rd Generation Partnership Project (3GPP)), Technical Report (TR) 38.802. Version 14.2.0 Available at https://portal.3gpp.org/desktopmodules/Specifications/ SpecificationDetails.aspx?specificationId=3066.

[17] 3GPP (2019) New WID: Further enhancements on MIMO for NR (3rd Generation Partnership Project (3GPP)), Work Item Description RP-193133. Available at https://portal.3gpp.org/ngppapp/CreateTDoc.aspx?mode=view\&contributionUid= RP-193133.

[18] 3GPP (2020) Evolved Universal Terrestrial Radio Access (E-UTRA); Radio Resource Control (RRC); Protocol specification (Release 16) (3rd Generation Partnership Project (3GPP)), Technical Specification (TS) 36.331. Version 16.2.0 Available at https://portal.3gpp.org/desktopmodules/Specifications/SpecificationDetails. aspx? specificationId=2440.

[19] Cintron FJ (2018) Performance evaluation of LTE device-to-device out-of-coverage communication with frequency hopping resource scheduling (National Institute of Standards and Technology, Gaithersburg, MD), NIST IR 8220. https://doi.org/10. 
6028/NIST.IR.8220

[20] 3GPP (2020) NR; User Equipment (UE) radio access capabilities (Release 16) (3rd Generation Partnership Project (3GPP)), Technical Specification (TS) 38.306. Version 16.3.0 Available at https://portal.3gpp.org/desktopmodules/Specifications/ SpecificationDetails.aspx?specificationId=3193.

[21] 3GPP (2018) Discussion on UE peak data rate (3rd Generation Partnership Project (3GPP)), TDoc R1-1803648. Available at https://portal.3gpp.org/ngppapp/ CreateTdoc .aspx $?$ mode $=$ view \& contributionId $=879805$.

[22] 3GPP (2020) NR; NR and NG-RAN Overall Description; Stage 2 (Release 16) (3rd Generation Partnership Project (3GPP)), Technical Specification (TS) 38.300. Version 16.2.0 Available at https://portal.3gpp.org/desktopmodules/Specifications/ SpecificationDetails.aspx?specificationId $=3191$.

[23] 3GPP (2020) System architecture for the 5G System (5GS); Stage 2 (Release 16) (3rd Generation Partnership Project (3GPP)), Technical Specification (TS) 23.501. Version 16.7.0 Available at https://portal.3gpp.org/desktopmodules/Specifications/ SpecificationDetails.aspx? specificationId $=3144$.

[24] 3GPP (2020) Policy and charging control architecture (Release 16) (3rd Generation Partnership Project (3GPP)), Technical Specification (TS) 23.203. Version 16.2.0 Available at https://portal.3gpp.org/desktopmodules/Specifications/ SpecificationDetails.aspx? specificationId $=810$.

[25] 3GPP (2020) E-UTRA and NR; Service Data Adaptation Protocol (SDAP) specification (Release 16) (3rd Generation Partnership Project (3GPP)), Technical Specification (TS) 37.324. Version 16.2.0 Available at https://portal.3gpp.org/desktopmodules/ Specifications/SpecificationDetails.aspx?specificationId=3282.

[26] 3GPP (2020) Architecture enhancements for 5G System (5GS) to support Vehicle-toEverything (V2X) services (Release 16) (3rd Generation Partnership Project (3GPP)), Technical Specification (TS) 23.287. Version 16.5.0 Available at https://portal.3gpp. org/desktopmodules/Specifications/SpecificationDetails.aspx? specificationId=3578.

[27] 3GPP (2020) Architecture enhancements for V2X services (Release 16) (3rd Generation Partnership Project (3GPP)), Technical Specification (TS) 23.285. Version 16.4.0 Available at https://portal.3gpp.org/desktopmodules/Specifications/ SpecificationDetails.aspx?specificationId=3078.

[28] 3GPP (2020) LS on new PQI support for PC5 communication (3rd Generation Partnership Project (3GPP)), Liaison Statement R2-2008761. Available at https://portal. 3gpp.org/ngppapp/CreateTdoc.aspx ?mode=view\&contributionId=1157530.

[29] 3GPP (2021) NG-RAN; Xn application protocol (XnAP) (Release 16) (3rd Generation Partnership Project (3GPP)), Technical Specification (TS) 38.423. Version 16.4.0 Available at https://portal.3gpp.org/desktopmodules/Specifications/ SpecificationDetails.aspx? specificationId $=3228$.

[30] MediaTek (2020) 5G NR and 4G LTE Coexistence; A Comprehensive Deployment Guide to Dynamic Spectrum Sharing, . White paper. Available at https://d86o2zu8ugzlg.cloudfront.net/mediatek-craft/documents/ 
Dynamic-Spectrum-Sharing-WhitePaper-PDFDSSWP-031320.pdf.

[31] 3GPP (2020) Release 17 timeline agreed. Press Release. Available at https://www. 3gpp.org/news-events/2145-rel-17_newtimeline.

[32] 3GPP (2013) Feasibility study for Proximity Services (ProSe) (3rd Generation Partnership Project (3GPP)), Technical Report (TR) 22.803. Version 12.2.0 Available at https://portal.3gpp.org/desktopmodules/Specifications/SpecificationDetails. aspx? specificationId=653.

[33] 3GPP (2019) Study on Network Controlled Interactive Services (3rd Generation Partnership Project (3GPP)), Technical Report (TR) 22.842. Version 17.2.0 Available at https://portal.3gpp.org/desktopmodules/Specifications/SpecificationDetails. aspx? specificationId=3521.

[34] 3GPP (2019) LS on D2D enabler for public safety communications in 5GS (3rd Generation Partnership Project (3GPP)), Liaison Statement S6-190280. Available at https://portal.3gpp.org/ngppapp/CreateTDoc.aspx?mode=view\&contributionUid= S6-190280.

[35] 3GPP (2019) Revised SID: Study on System enhancement for Proximity based Services in 5GS (3rd Generation Partnership Project (3GPP)), Work Item Description SP190443. Available at https://portal.3gpp.org/ngppapp/CreateTdoc.aspx ?mode=view\& contributionUid=SP-190443.

[36] 3GPP (2021) Study on system enhancement for Proximity based Services (ProSe) in the 5G System (5GS) (Release 17) (3rd Generation Partnership Project (3GPP)), Technical Report (TR) 23.752. Version 17.0.0 Available at https://portal.3gpp.org/ desktopmodules/Specifications/SpecificationDetails.aspx ? specificationId=3624.

[37] 3GPP (2021) Study on NR sidelink relay (Release 17) (3rd Generation Partnership Project (3GPP)), Technical Report (TR) 38.836. Version 17.0.0 Available at https://portal.3gpp.org/desktopmodules/Specifications/SpecificationDetails. aspx? specificationId $=3725$.

[38] 3GPP (2021) Revised WID on System enhancement for Proximity based Services in 5GS (3rd Generation Partnership Project (3GPP)), Work Item Description SP-210272. Available at https://portal.3gpp.org/ngppapp/CreateTDoc.aspx?mode= view\&contributionUid=SP-210272.

[39] 3GPP (2021) New WID on NR Sidelink Relay (3rd Generation Partnership Project (3GPP)), Work Item Description RP-210904. Available at https://portal.3gpp.org/ ngppapp/CreateTDoc.aspx? mode=view \&contributionUid=RP-210904.

[40] 3GPP (2021) Proximity based Services (ProSe) in the 5G System (5GS) Release 17 (3rd Generation Partnership Project (3GPP)), Technical Specification (TS) 23.304. Version 0.1.0 Available at https://portal.3gpp.org/desktopmodules/Specifications/ SpecificationDetails.aspx? specificationId $=3855$.

[41] 3GPP (2020) WID revision: NR Sidelink enhancement (3rd Generation Partnership Project (3GPP)), Work Item Description RP-202846. Available at https://portal.3gpp. org/ngppapp/CreateTDoc.aspx?mode=view\&contributionUid=RP-202846.

[42] 3GPP (2019) Summary of email discussion on Rel-17 sidelink enhancement (3rd 
Generation Partnership Project (3GPP)), Tdoc RP-192745. Available at https://www. 3gpp.org/ftp/tsg_ran/TSG_RAN/TSGR_86/Docs/RP-192745.zip.

[43] 3GPP (2019) New SID: Study on architecture enhancements for 3GPP support of advanced V2X services - Phase 2 (3rd Generation Partnership Project (3GPP)), Work Item Description SP-190631. Available at https://portal.3gpp.org/ngppapp/ CreateTdoc .aspx ?mode=view\&contributionUid=SP-190631.

[44] 3GPP (2020) Study on architecture enhancements for 3GPP support of advanced Vehicle-to-Everything (V2X) services; Phase 2 (3rd Generation Partnership Project (3GPP)), Technical Report (TR) 23.776. Version 1.0.0 Available at https://portal.3gpp.org/desktopmodules/Specifications/SpecificationDetails. aspx? specificationId=3706.

[45] 3GPP (2020) Revised SID on enhancements to application layer support for V2X services (3rd Generation Partnership Project (3GPP)), Work Item Description SP200110. Available at https://portal.3gpp.org/ngppapp/CreateTdoc.aspx ?mode=view \& contributionUid=SP-200110.

[46] 3GPP (2020) Study on enhancements to application layer support for V2X services; (3rd Generation Partnership Project (3GPP)), Technical Report (TR) 23.764. Version 17.1.0 Available at https://portal.3gpp.org/desktopmodules/Specifications/ SpecificationDetails.aspx?specificationId $=3658$.

[47] 3GPP (2020) New WID for enhanced application layer support for V2X services (3rd Generation Partnership Project (3GPP)), Work Item Description SP-200831. Available at https://www.3gpp.org/ftp/tsg_sa/TSG_SA/TSGs_89E_Electronic/Docs/ SP-200831.zip. 